DOE NERI

DE-FC07-05ID14649

\title{
The Development of Models to Optimize Selection of Nuclear Fuels through Atomic-Level Simulation
}

\author{
PI: Professor Simon R. Phillpot \\ Department of Materials Science and Engineering \\ University of Florida \\ Gainesville FL 32611 \\ Phone: (352) 846-3782 \\ Fax: (352) 846-3355 \\ sphil@mse.ufl.edu \\ Co-PIs: \\ Prof. Susan B. Sinnott, Department of Materials Science and Engineering \\ Prof. Hans Seifert, Department of Materials Science and Engineering \\ Prof. James S. Tulenko, Department of Nuclear and Radiological Engineering
}

Report for April 152005 - October 152008

Final Report 


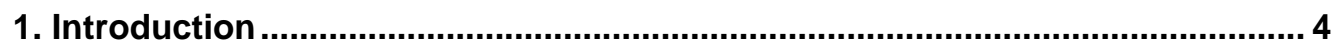

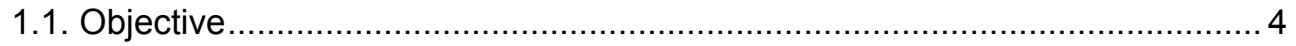

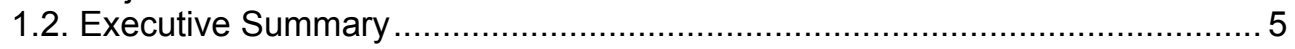

2. Integration of First Principles Methods into the FRAPCON Fuel-Performance

Code

3. Molecular-Dynamics Simulations .................................................................... 18

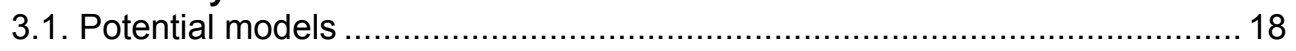

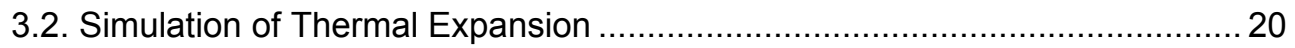

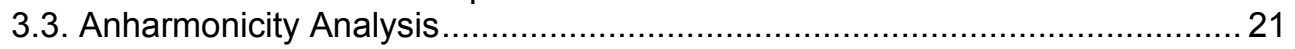

3.4. Thermal Conductivity of Single Crystal .................................................. 22

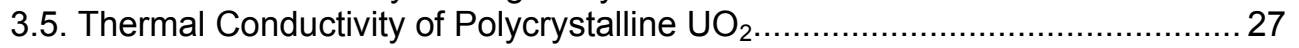

3.5.1. Structure of Model Polycrystal .............................................................. 27

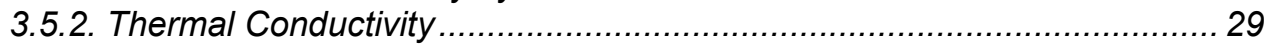

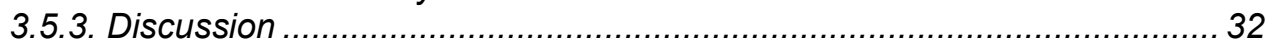

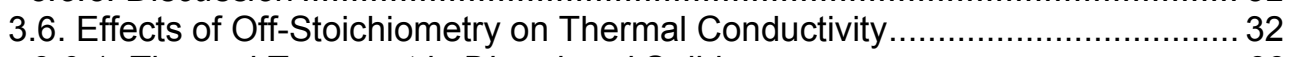

3.6.1. Thermal Transport in Disordered Solid .................................................. 33

3.6.2. Thermal Transport in $\mathrm{UO}_{2}$ with Point Defects ...................................... 33

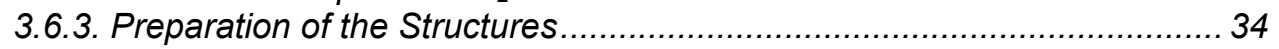

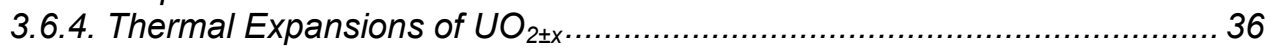

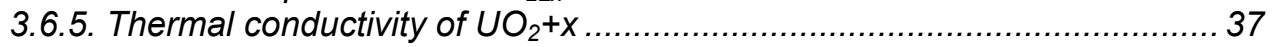

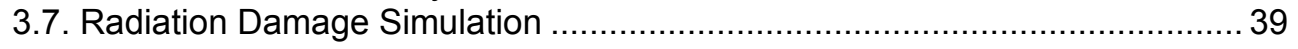

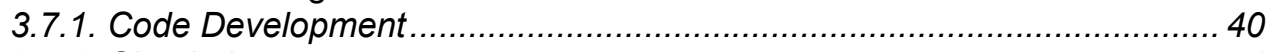

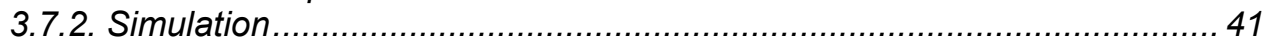

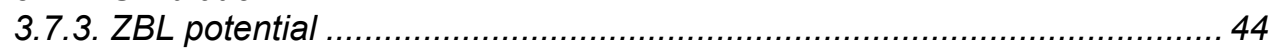

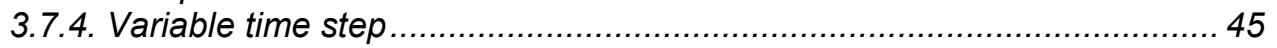

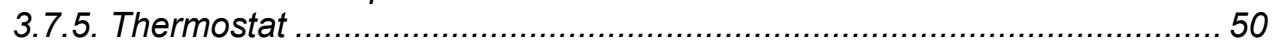

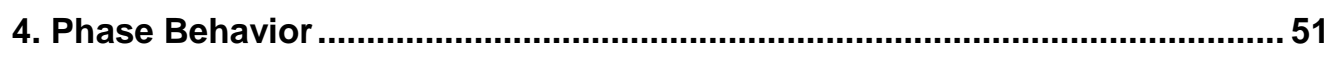

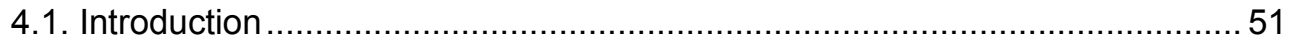

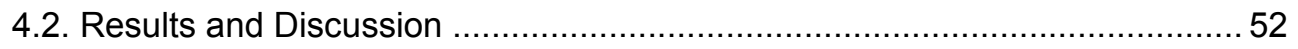

4.2.1. Thermodynamic Optimization of the Ce-O System .............................. 52

4.2.2. Critical assessments (evaluations) of nuclear materials systems ............. 55 
4.2.3. Experimental literature data for the U-Pu-O system ............................. 56

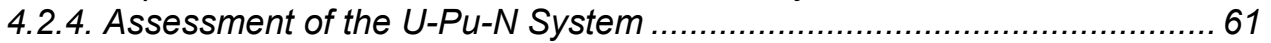

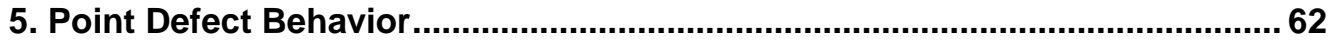

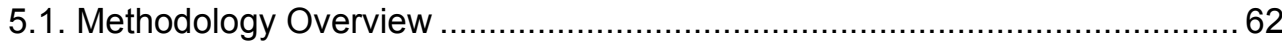

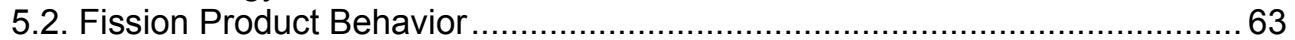

6. Papers, Reports, Theses and Presentations ..................................................... 66 


\section{Introduction}

\subsection{Objective}

The objective of this project was to take the first steps in developing an advanced fuel performance platform, based on the FRAPCON code, with detailed materials input from databases of nuclear materials behavior determined from thermodynamic, atomic-level and electronic-level calculations and simulations.

Fuel performance codes calculate steady state parameters such as fuel temperatures and stored energy, fuel rod internal pressures, irradiation growth, cladding oxidation, cladding stresses and strains, and the rod power needed to lead to a melt condition. Currently all fuel performance codes are based on correlations developed by fitting experimental data to the variables involved. These variables include material composition, temperature, density, closed porosity, surface roughness, initial size of fuel grains, sintering temperature of the fuel, roughness of the fuel surface, and fractional cold work of the cladding. The challenge is that these correlations do not work for operating conditions that exceed those experimental points that are serving as the basis for the correlation. Moreover, the fuel performance codes cannot be used for materials for which no experimental correlations exist. It is the objective of the UF team to see in what areas performance codes can be extended to new materials based on databases developed from the thermodynamic, atomistic and first principles calculations.

The program consists of dissecting and then rebuilding the FRAPCON code into a form that can use materials databases developed from atomic-level, electronic-level and thermodynamic calculations and to use sensitivity analysis to identify the materials properties on which fuel performance is most dependent.

The resulting code serves as a prototype for a new generation of advanced fuel performance codes with inputs from state-of-the-art electronic-structure, atomic and thermodynamic calculations.

The UF team consisted of four experts with complementary skills in nuclear fuels and materials issues. As a result of support from this program three students will have received Ph.D., and two will have received M.S. degrees. These students will be well-positioned to become technical leaders in a wide variety of issues related to nuclear fuel problems. Indeed one of the M.S. students (Daniel Vega) is now a program officer with DOE-NE.

Development of a fully "first principles" based code will allow fuel development programs to move from the costly and time consuming "Cook and Look" philosophy of testing each fuel type to a modeling, simulation, analysis, and selection process, that will require irradiations only in a confirmatory mode. 


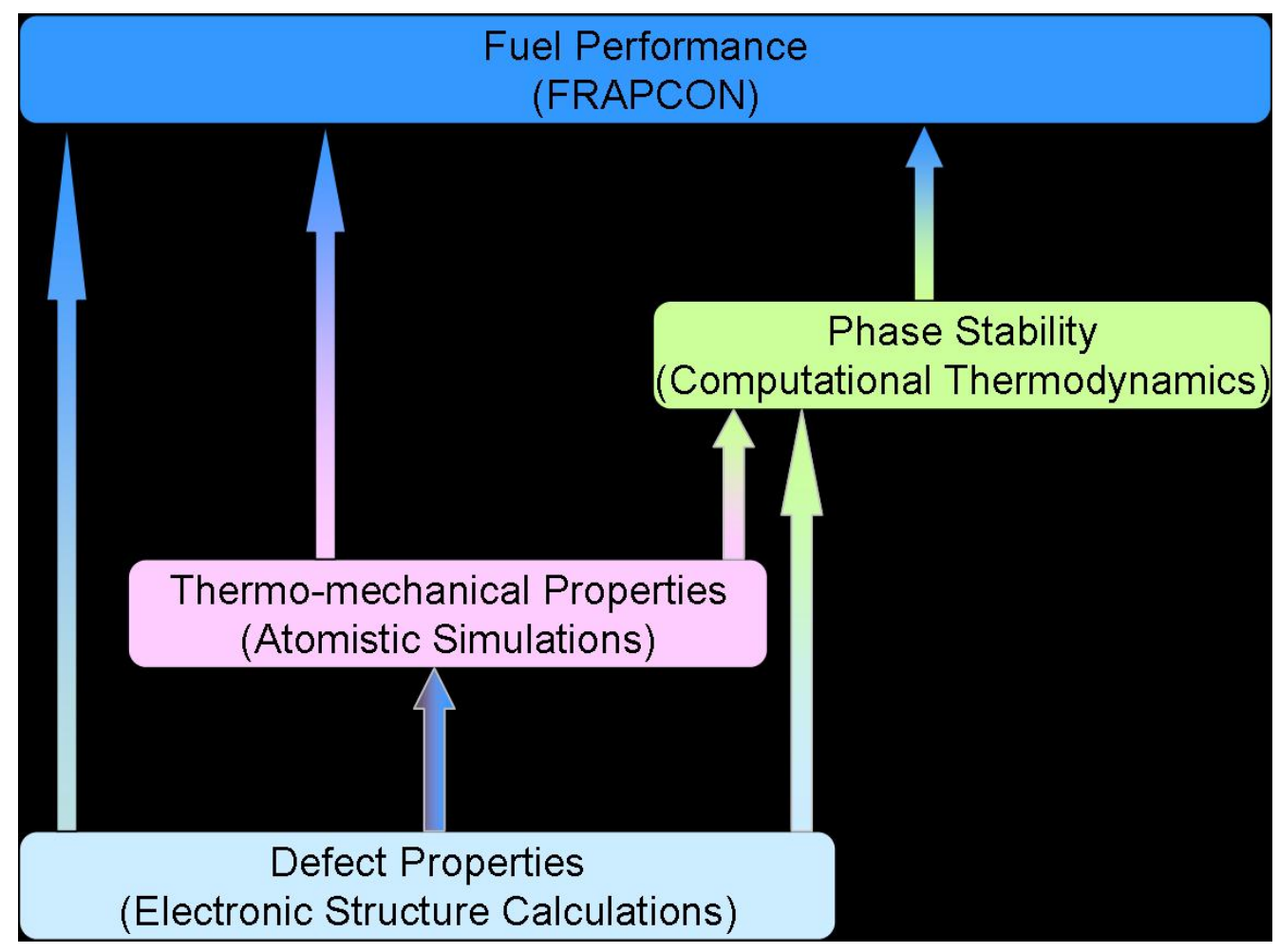

Figure 1-1. Schematic showing the four tasks in this project and the links between them.

\subsection{Executive Summary}

\section{5}

Notification of the award of this proposal was received in December 2004; the program began on April $15^{\text {th }} 2005$ and was completed October 15th 2008. The work has been executed by the UF team in close collaboration with colleagues at Los Alamos National Lab, Idaho National Lab, and Imperial College, London.

The technical accomplishments under this program include:

- Demonstrated that FRAPCON can be modified to accept data generated from first principles studies, and that the result obtained from the modified FRAPCON make sense in terms of the inputs

- Determined the temperature dependence of the thermal conductivity of single crystal $\mathrm{UO}_{2}$ from atomistic simulation. 
- Evaluated the temperature and grain-size dependence of the thermal conductivity of polycrystalline $\mathrm{UO}_{2}$ from atomistic simulation. Determination of the interfacial resistance for a typical $\mathrm{UO}_{2}$ grain boundary.

- Determined the temperature and composition dependence of the thermal conductivity of hypo-and hyper-stoichiometric $\mathrm{UO}_{2}$ from atomistic simulation. Analysis of the thermal conductivity in terms of the thermal-transport behavior of amorphous systems

- Developed new methodologies for the simulation of radiation damage in oxides.

- Performed a thermodynamics analysis of the Ce-O system

- Performed critical assessments of the thermodynamics phase diagrams of the $\mathrm{U}-\mathrm{Pu}-\mathrm{O}$ and U-Pu-N systems

- Established the energetics associated with intrinsic defects in UO2 using a combination of state-of-the-art electronic structure calculations and a powerful thermodynamic analysis

- Determined the solution and incorporation energies of key soluble and nonsoluble fission products.

This program has enabled a significant amount of graduate student education, leading to 2 M.S. degrees and 3 Ph.D. degrees:

- Mr. Christian Acosta began the work on the FRAPCON code with Prof. Tulenko. He has since graduated with an M.S. degree.

- Mr. Daniel A. Vega carried on the FRAPCON work, receiving an M. S. degree in Summer 2008

- Mr. Taku Watanabe worked with Profs. Phillpot and Sinnott on the use of atomic-level simulation methods to determine the thermal transport properties of UO2. He received his Ph.D. in Spring 2008.

- Mr. Pankaj Nerikar is working with Profs. Sinnott, Phillpot and Seifert. Initially, his work was on thermodynamic calculations of the U-Pu-O and U-Pu-N systems; in the latter part of the program he worked on using electronic-structure methods to characterize the defect behavior in $\mathrm{UO}_{2}$. He will receive his Ph.D. in Spring 2009

- Mr. Dilpuneet Aidhy has worked with Dr. Phillpot on the simulation of radiation damage in $\mathrm{UO}_{2}$ and related oxides. He will receive his Ph.D. in Spring 2009.

Three of these students made extended research visits to our collaborators in national laboratories:

- Taku Watanabe spent four months over the summer of 2006 in MST-8 at Los Alamos National Laboratory working with Drs. Blas Uberuaga, Marius Stan, Srini Srivilliputhur and Art Voter on cascade simulations in $\mathrm{UO}_{2}$ and on point defect calculations.

- Dilpuneet Aidhy spend a year (2007-2008) working on radiation-damage simulations with Dr. Dieter Wolf in the Materials Sciences Division at Idaho National Laboratory. 
- Pankaj Nerikar is spending a year working with Dr. Blas Uberuaga at LANL on pointdefect energetics in $\mathrm{UO}_{2}$.

\section{Integration of First Principles Methods into the FRAPCON Fuel- Performance Code}

The two primary tasks of this part of the project were to rebuild the FRAPCON fuel performance code so that it can accept information from databases including first-principles results, and to produce databases of the U-Pu-O and U-Pu-N system properties. The restructuring of the code involved performing a detailed review of the original code, performing a sensitivity analysis to determine the key physical variables, and implementing the use of database material within the code itself. The elements of the FRAPCON code that are important to temperature calculations were reviewed and the subroutines needed were modified in order to accomplish the rebuilding process to incorporate the first-principles data. Using the information presented in the FRAPCON review, the sensitivity analysis was completed in a timely fashion and is being published in the Journal of Nuclear Technology.

\subsection{FRAPCON Code}

FRAPCON-3 is a computer code for the calculation of a steady-state thermo-mechanical behavior of oxide fuel rods sponsored by the U.S. Nuclear Regulatory Commission (NRC) and maintained by Pacific Northwest National Laboratory (PNL). When power and boundary conditions changes are sufficiently slow for "steady state" conditions to apply (such as constant power and slow power ramps), FRAPCON-3 uses a single rod analysis to calculate the variation over time of significant fuel rod variables, including fuel and cladding temperatures, cladding hoop strain, cladding oxidation, fuel irradiation swelling, fuel densification, fission gas release, and rod internal gas pressures. This analysis is used to both produce a time-step profile of thermo-mechanical properties, and to generate initial conditions for transient analysis.

The Nuclear Regulatory Commission Publication NUREG/CR-6534 describes specifics about the Thermo-Mechanical behavior of $\mathrm{UO}_{2}$ fuel rods modeled by FRAPCON. The unique heat transfer coefficient put forth by FRAPCON for the fuel section takes into account, not only the fuel's thermal conductivity, but also the gap size and fuel-cladding interfacial pressure on the overall conductivity of the fuel, using what is called the FRACAS-I, or rigid-pellet model.

FRAPCON-3, the latest release, uses the FRACAS- $1^{1,2}$ mechanical model, and the ANS $5.4^{1}$ fission gas release model, as well as a MATPRO ${ }^{3}$ materials properties package, modified to include high-burnup properties (see Fig. 2-1). These models are created from the analysis of spent fuel and will be discussed individually below.

While it should be noted that, as with any code, inherent limitations exist, for FRAPCON these limitations are well documented, and are more related to cladding behavior than fuel behavior, and are not necessarily pertinent to the immediate scope of this project. 


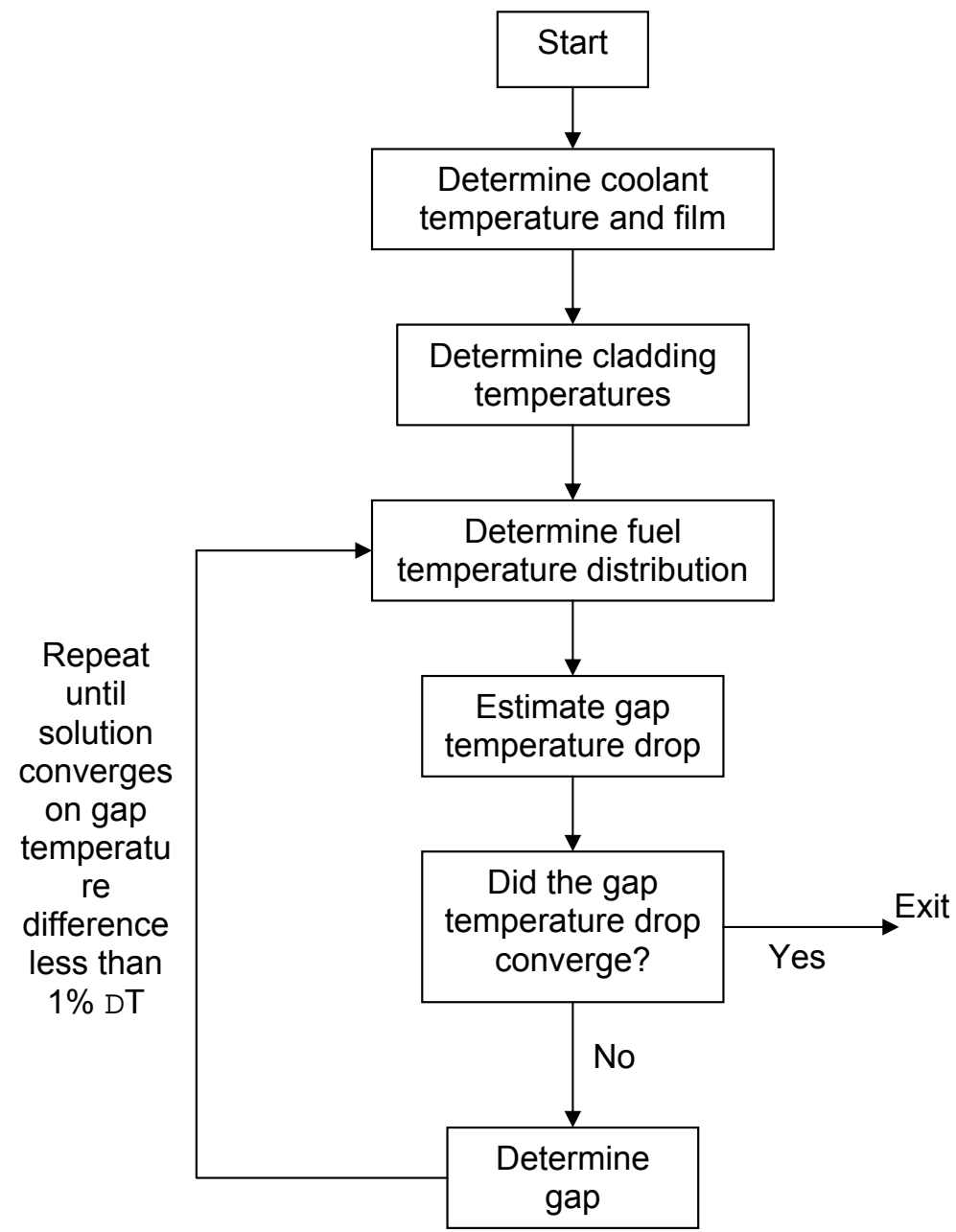

Figure 2-1. Flowchart of the Fuel Temperature Solution Method (reproduced from FRAPCON manual)

The main assumptions made in the FRAPCON code regarding the temperature distribution calculation are:

- Heat conduction in the axial direction is considered negligible

- Heat conduction in the azimuthal direction is negligible

- Boundary conditions are constant during each step

- Heat flow is steady state

\subsection{The FRACAS-I Model}


The FRACAS-I model is designed to handle fuel and cladding mechanical deformation in order to better simulate the effects of burn-up and temperature on the fuel pin. FRACAS can model two situations. The first is when the fuel and cladding are not in contact. This "open gap" situation has cladding with internal and external pressures that must be solved. The second situation is when the fuel has expanded enough to be in contact with the cladding. This "closed gap" situation has the fuel driving the cladding outwards. Overall, the calculations take into account the effects of fuel thermal expansion, fuel swelling, fuel densification, fuel relocation, cladding thermal expansion, cladding creep, cladding plasticity, and fission gas/external coolant pressures. For the purposes of this project, the main effect of the FRACAS-I model is to determine the gap thickness. .

\subsection{FRAPCON Modules}

As with most codes, FRAPCON runs a series of subroutines during its execution loops. Most of the subroutines did not need to be edited, and ran correctly when the code was edited. Those that were edited were tested for continued functionality, and continued to work properly.

FRAPCON has been rebuilt into a functioning workspace. The individual modules are directly accessible, and test calculations were performed. Sensitivity analysis at a basic level has also been performed -- specifically by recording the average fuel temperature, fission-gas release, burnup, heat transfer responses for variable and manually set conductivity values in the new module.

In its original state, as indicated above, the heat conduction coefficient for the fuel heat transfer is calculated using a five-factor formula:

$$
k=k_{o} * F D * F P * F M * F R
$$

$k_{o} \quad$ is thermal conductivity of un-irradiated urania

$F D \quad$ is the dissolved fission product factor

$F P \quad$ is the precipitated fission product factor

$F M \quad$ is the effect of porosity

$F R \quad$ is the radiation effect

The code was modified to permit an additional flag to be used to enter an additional module created specifically for this project. "fthcon.f" is the existing module used to calculate thermal conductivity. A newly created module is called from "fthcon.f"and returns heat conduction data to "fthcon.f, so that the returned heat conduction coefficient value is entered and used like any other conductivity value, and passed through the rest of the code normally.

The new module is a mirror of the existing algorithms used to compute the five-factor formula. Having been isolated from the rest of the code, however, this new module was ready to be modified for the current project.

The modifications to the fthcon subroutine are shown below in bold face.

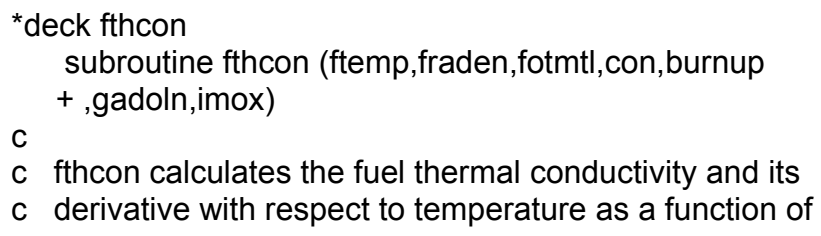




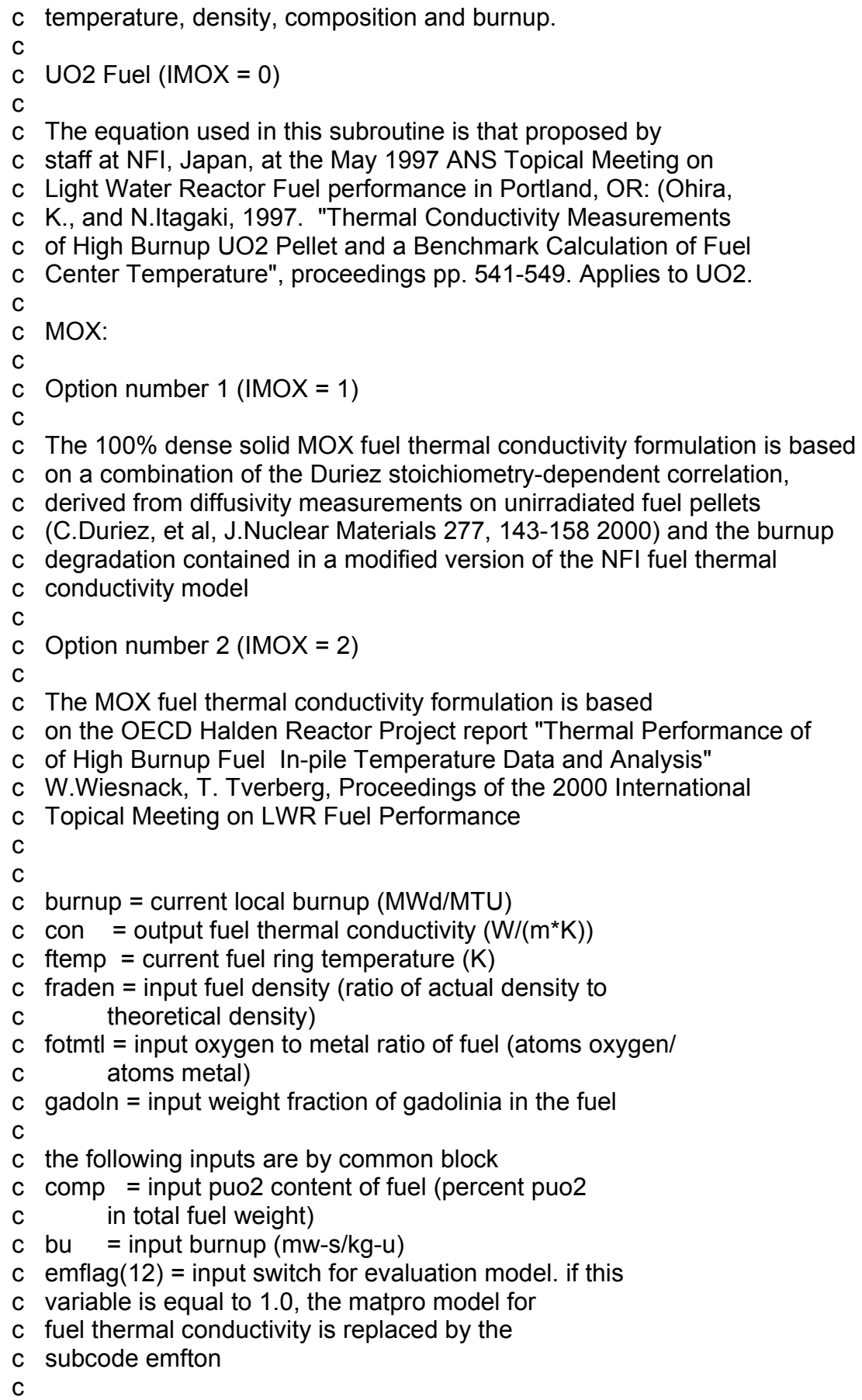

common / phypro / ftmelt, fhefus, ctmelt,chefus, ctranb, 


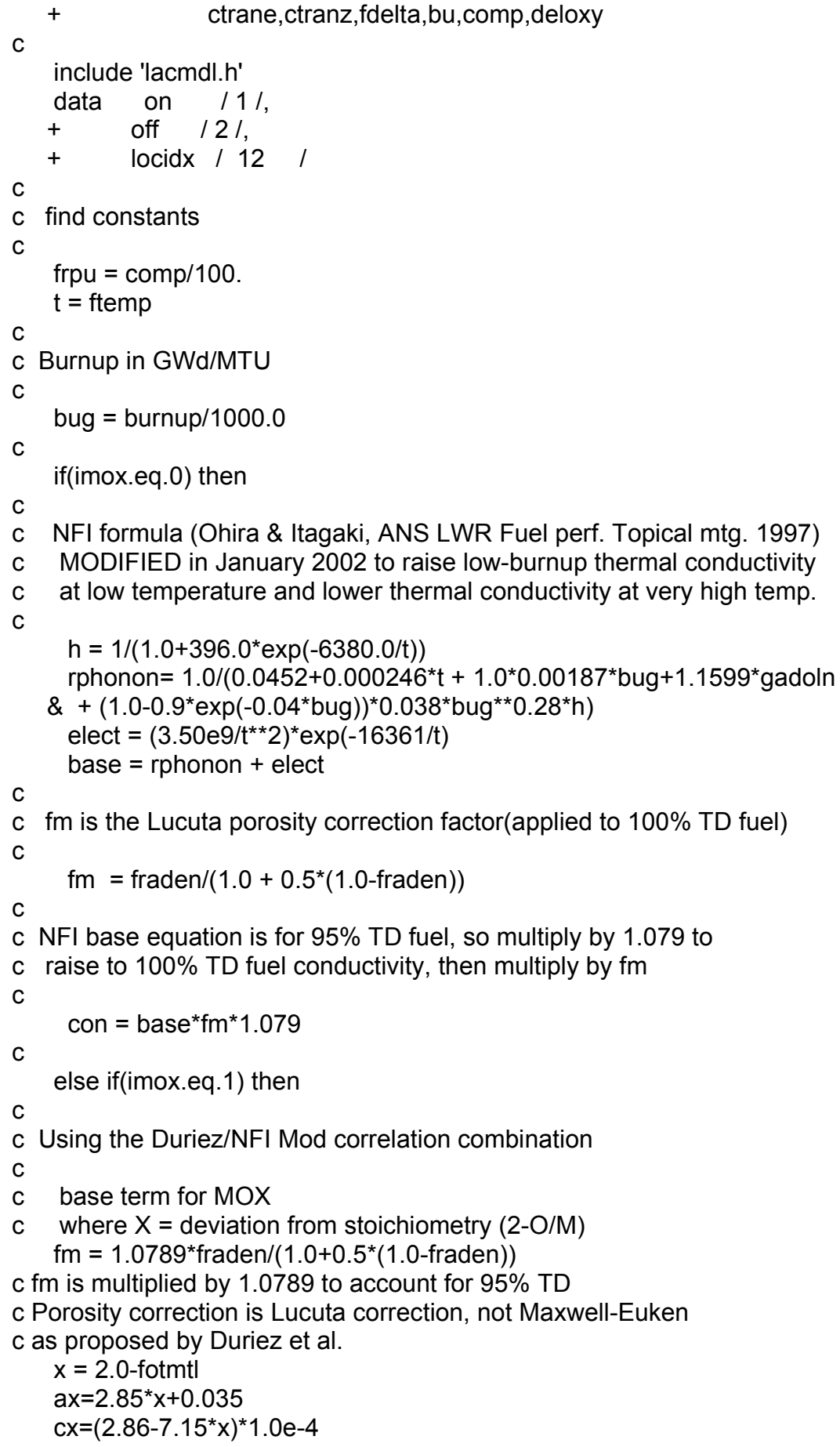




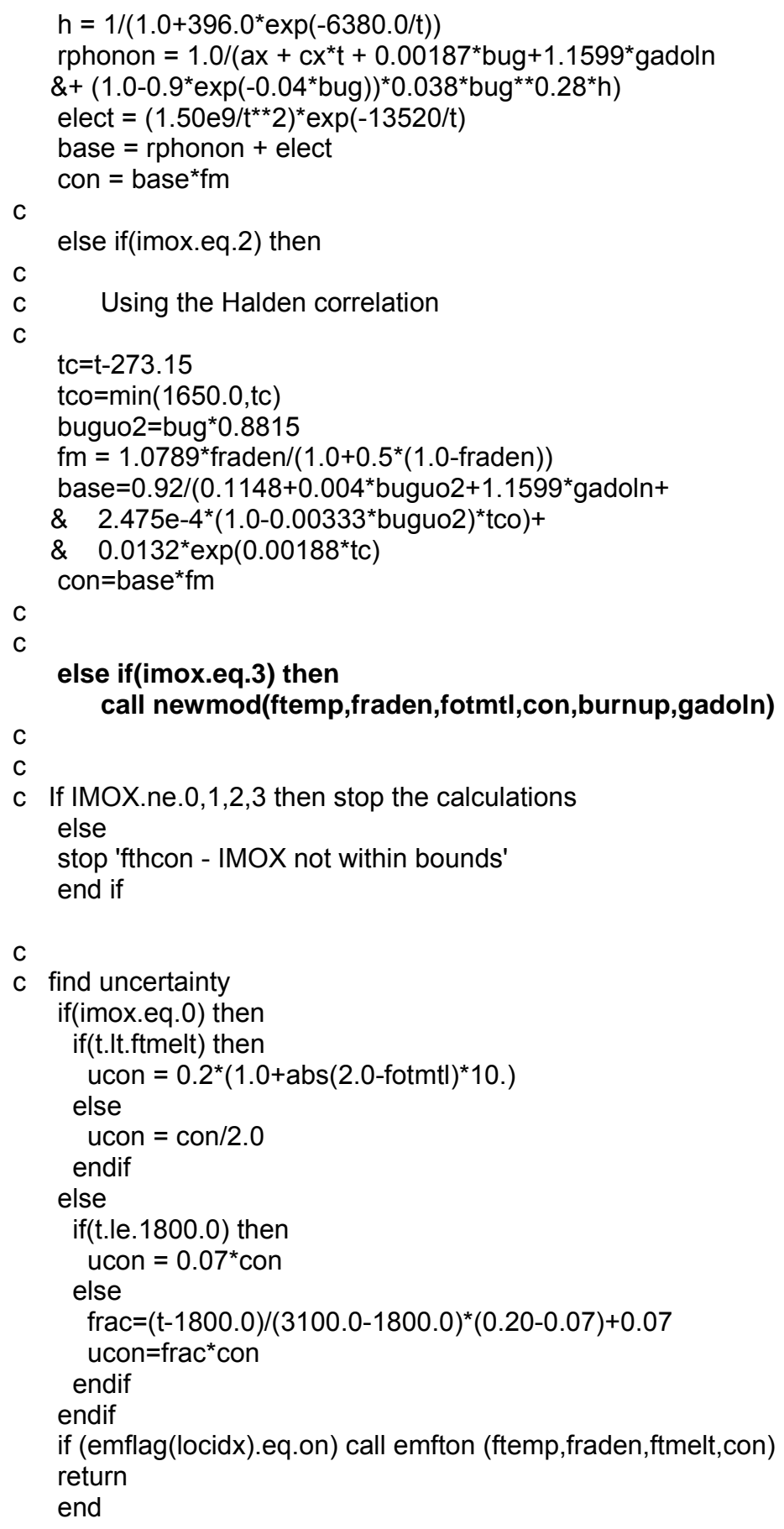




\subsubsection{Additions to FRAPCON Module}

The "fthcon.f" subroutine is fed information on the fuel temperature, fuel density, oxygen-to metal ratio, burnup, gadolinia weight fraction, and a flag called imox. This imox flag can now have 1,2 , or 3 as its value. This third value acts as a trigger that activates the new external module in which the new, first-principles derived, thermal conductivity data are included. The following examples assume that the new thermal conductivity values are not for MOX fuel.

In order to demonstrate the method by which new conductivity data is passed into the new module, a sample of the proposed model is given below:

\subsubsection{Newly Written Module}

include 'newmodule. $h$ '

*deck newmodule

subroutine newmodule (ftemp, fraden,fotmtl,con,burnup,gadoln)

real temp1,con1,temp2,con2

open ( $u$, FILE='contable.txt', STATUS='OLD')

c in the open statement " $u$ " is an identifier that needs to be given as a number between 0

c 99 and must be unique.

$\operatorname{read}\left(5,{ }^{*}\right)$ temp $1, \operatorname{con} 1$

$90 \operatorname{read}\left(5,{ }^{*}\right)$ temp2,con2

c this reads the first two values from the input stream in logical unit 5 and assigns them

$\mathrm{c}$ to variables temp1 and con1. Assuming variables are entered in the order they appear

c in the subroutine call, temp1 is the fuel temperature and con1 is the thermal

c conductivity. This process is repeated for the next temperature entry in the table.

if(temp1.GE.ftemp) go to 100

c this checks to see if our fuel temperature is less than the smallest temperature in the

c table. In this case, the smallest temperature thermal conductivity is returned.

if(temp2.GE.ftemp) go to 110

c this checks to see if the fuel temperature is less than the next temperature entry in the

c table. If it is, that means it lies between the first and second entries. The program than

c interpolates linearly and returns a thermal conductivity.

temp1 $=$ temp2

$\operatorname{con} 1=\operatorname{con} 2$

go to 90

c assuming the other conditions failed, we need to advance in the table. This sets the

C second point as the first point and repeats the loop from the beginning. 
100 con $=$ con 1

go to 120

110 con $=($ ftemp-temp 1$) /($ temp2-temp 1$) *($ con2-con 1$)+$ con 1

120 return

end

\subsection{FRAPCON code Modifications}

\subsubsection{Thermal Conductivity}

The "base case" system from the unmodified FRAPCON was characterized and archived. The base case serves as the basis for comparison of individual thermo-mechanical behavior, as well as overall fuel performance characterization. It consists of a standard fuel element from Oconee Nuclear Station near Greenville, SC.

The MD model (Section 3) has generated atomic level thermo-mechanical data and independent relationships to describe the thermal conductivity as a function of temperature for a fresh, beginning of life (BOL) fuel element as given below:

$$
\begin{array}{clrl}
\text { MD : } & k= & 59848 \cdot T^{-1.291} \\
\text { MATPRO }: & k=\frac{1}{0.0452+0.000246 T}+\frac{3.50 \times 10^{9}}{T^{2}} \exp \left(-\frac{16361}{T}\right)
\end{array}
$$

Using these correlations, FRAPCON and the MD models exhibited showed very good agreement for the beginning of life temperature profile through the pellet, as shown in Figure. $2-2$.

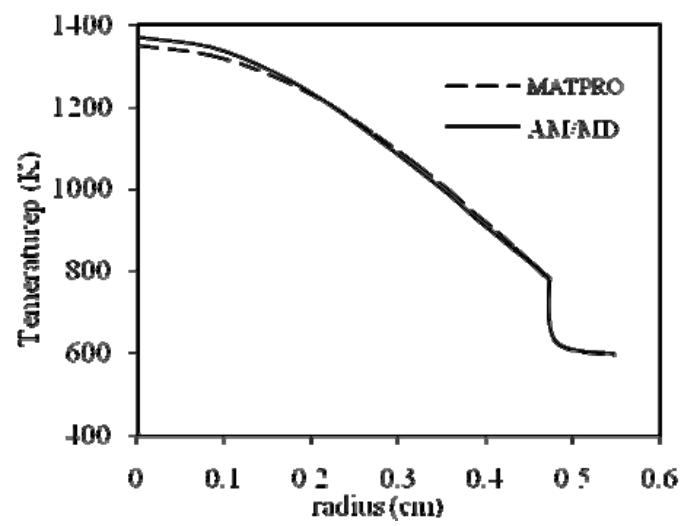

Figure 2-2. Radial Temperature Profile of Fuel Pellet

\subsubsection{Thermal Expansion}

The fuel deformation analysis in FRAPCON includes the effects of fuel thermal expansion, swelling, densification, and relocation. The base case modeling regime includes an elasticplastic uni-axial stress/strain analysis given by the following equation: 


$$
\varepsilon_{i j}=\frac{1+v}{E} \sigma_{i j}-\delta i j\left(\frac{v}{E} \sigma_{i j}-\int \alpha \mathrm{d} T\right)+\varepsilon_{i j}+d \varepsilon_{i j}
$$

FRAPCON thermal expansion modes have been isolated within the code, and several versions have been created to compare beginning of life (BOL) thermal expansion data. These versions consist of Fink's Model (an empirical fit to experimental data), the results from the MD simulations described in Section 3, and the empirical fit used as a base in FRAPCON. As a measure of thermal expansion, the fuel pellet surface displacement and surface axial strain were compared for the models. Table 2-1 provides a list of the surface displacement at BOL.

Table 2-1. Axial node 4/12, 800K, 0 Burnup

\begin{tabular}{|c|c|c|}
\hline Model & Pellet Surface Displacement (mils) & Thermal Expansion (mils) \\
\hline MD & 3.192 & 1.648 \\
\hline MATPRO & 2.945 & 1.401 \\
\hline
\end{tabular}

The current models are summarized below:

Fink: $\quad \alpha(T)=9.828 \times 10^{-6}-6.930 \times 10^{-10} T+1.330 \times 10^{-12} T^{2}-1.757 \times 10^{-17} T^{3}$

MD: $\quad \alpha(T)=\quad 8.835 \times 10^{-6}(1+5.467 T)$

MATPRO: $\alpha(T)=-3.0 \times 10^{-3}+1.0 \times 10^{-5} T+4.0 \times 10^{-2} \exp \left(6.9 \times 10^{-12} / k_{B} T\right)$

The resulting strain curves are given in Figure. 2-3.

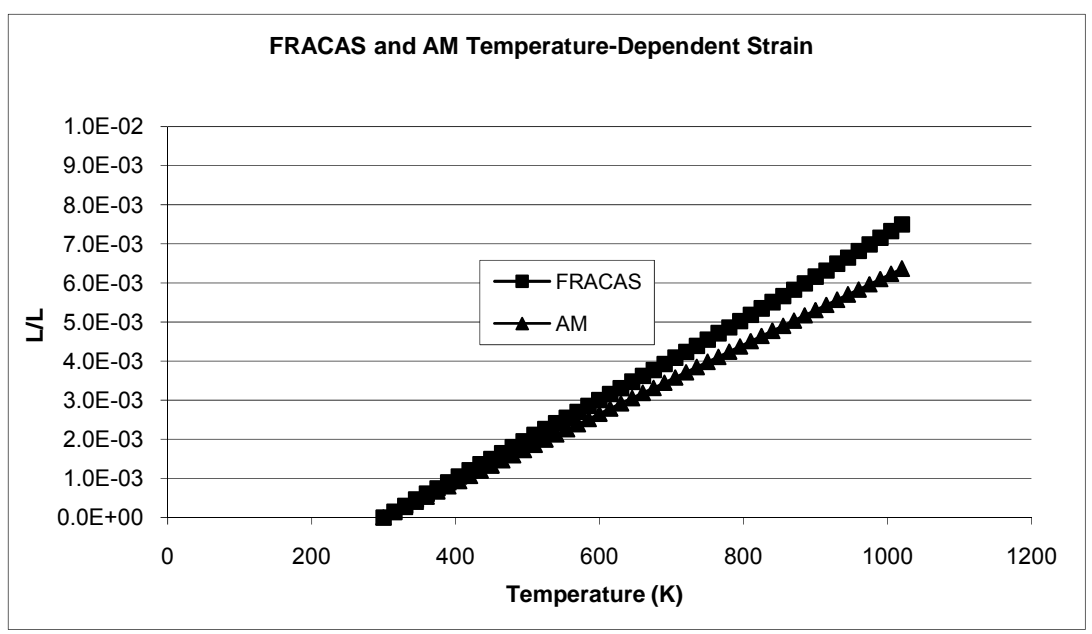

Figure 2-3. FRACAS and MD/AM Temperature-dependent strain plot 
The relatively poor predictions for the thermal expansion are primarily a result of the rather less than optimal materials fidelity of the interatomic potential used in the AM/MD simulations.

AM/MD simulations show that the predicted thermal expansion (and associated thermal strain) does not change to any great extent for systems with point defects or polycrystalline microstructures. This is consistent with the FRACAS assumption of the independence of the thermal expansion on microstructure, and with experimental results on a wide range of materials. These FRACPON and AM/MD models for the thermal strain of unirradiated $(\mathrm{BOL}) \mathrm{UO}_{2}$ are compared in Figure. 3. This plot shows that the two models exhibit similar behavior, but are not in quantitative agreement. This disagreement notwithstanding, it is useful to carry out a preliminary analysis of the effect of the thermal expansion model on the evolution of the system during burnup. The results of a FRAPCON calculation of the surface displacement due to thermal and burnup effects over time are given in Figure 2-5.

As is evident in Figure. 2-4, the first 100 days are very nearly identical in the FRACAS and $\mathrm{AM} / \mathrm{MD}$ models. After the first 100 days, the AM model significantly underestimates the fuel pellet dilatation, but still exhibits a similar shape. After approximately 1000 days, the AM and FRACAS models converge. This agreement arises from the closing of the gap between the fuel and the cladding, thereby inhibiting the thermal expansion. As a result the details of the thermal-expansion model become unimportant.

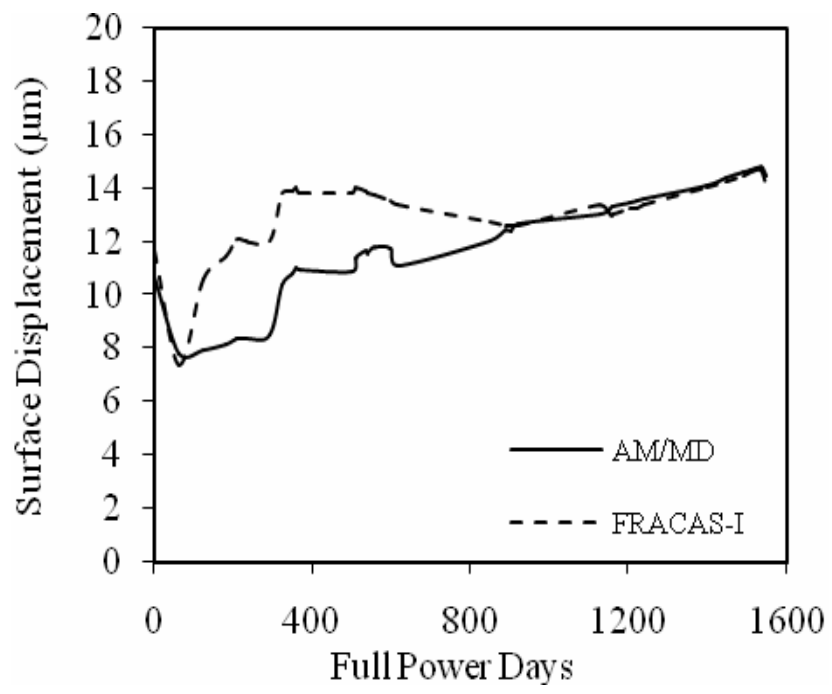

Figure 2-4. Lifetime Surface Displacement for Time-dependent Phenomena

\subsection{Section Conclusions}

In summary, this part of the program has been successful in providing a proof-of-principle that a fuel performance code can be adapted to accept input from atomistic model simulations. A very significant amount of further research is, however, required before atomistic models can reliably supplement, let alone overtake accuracy obtained by detailed 
experiments. The direct comparisons with base FRAPCON results show current AM/MD models cannot yet always provide inputs of sufficient materials fidelity to be quantitatively predictive. Such materials fidelity is only achievable by continued research in improvement of interatomic potentials.

The two properties addressed in this investigation are likely the most simple to integrate into a fuel performance code, but are in addition the most important. The sizable challenge faced in developing an atomistically informed fuel-performance code is that of incorporating the complexities associated with the changing chemistry associated with such effects as fuel swelling, fuel densification, fuel relocation, cladding thermal expansion, cladding creep, cladding plasticity, and fission gas release.

\subsection{FRAPCON code modifications}

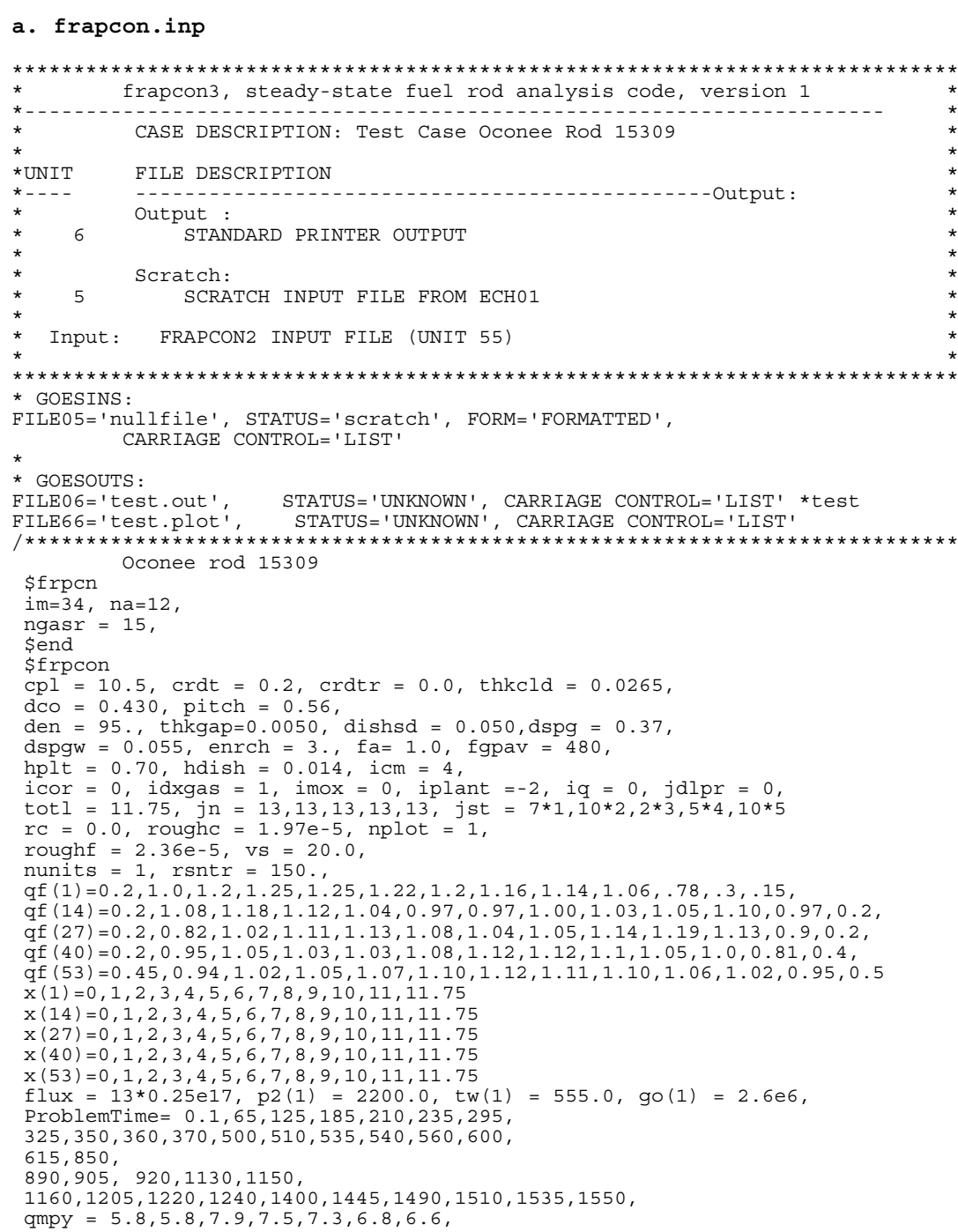


$7.9,7.6,7.4,6.9,6.6,6.1,6.7,6.0,6.6,6.1$,

$4.1,5.4$,

$5.1,4.7,5.4,5.0,4.5$,

$4.3,4.4,4.3,4.4,4.5,4.55,4.6,4.65,4.7,3.6$,

slim $=.05$

$\$ \mathrm{e}$

\section{Molecular-Dynamics Simulations}

Molecular dynamics (MD) is particularly useful in the study of nonequilibrium dynamic processes at the atomic level. MD treats atoms as point particles with no internal structure. For each atom, mass and charge are assigned. All atoms are treated classically and their dynamics are obtained by solving the Newton's equation of motion. For that, the only physical input required by the MD simulation is the interatomic potential.

\subsection{Potential models}

The interatomic interactions consist of a long-range electrostatic component and short-range interactions which describe the materials specific largely-repulsive component. In this work, the results obtained from two short-range interaction models: those due to Yamada ${ }^{4}$ and the Busker $^{5,6}$ The Busker potential is a traditional Buckingham potential, which is the most commonly used interaction model for ionic materials:

$$
\phi\left(r_{i j}\right)=A_{i j} \exp \left(-r_{i j} / \rho_{i j}\right)-\frac{c_{i j}}{r_{i j}{ }^{6}}
$$

Here $r_{i j}$ is the interatomic distance between atom $i$ and $j . A_{i j}, \rho_{i j}$, and $c_{i j}$ are the fitting parameters between each atomic species. The values of the parameters are given in Table 3-1.

Table 3-1. Parameters of interatomic potentials

\begin{tabular}{|c|c|c|c|c|c|c|c|}
\hline & \multicolumn{3}{|c|}{ Busker } & \multicolumn{3}{|c|}{ Yamada } \\
\hline & & $\mathrm{U}-\mathrm{U}$ & $\mathrm{O}-\mathrm{O}$ & $\mathrm{U}-\mathrm{O}$ & $U-U$ & O-O & $\mathrm{U}-\mathrm{O}$ \\
\hline $\mathrm{A}_{\mathrm{ij}}$ & {$[\mathrm{eV}]$} & 0 & 9547.96 & 1761.775 & 442.208 & 2346.149 & 1018.571 \\
\hline$\rho_{i j}$ & {$[\AA]$} & 0 & 0.2192 & 0.35643 & 0.32 & 0.32 & 0.32 \\
\hline$C_{i j}$ & {$\left[e V \cdot \AA^{6}\right]$} & 0 & 32 & 0 & 0 & 4.1462 & 0 \\
\hline$D_{i j}$ & {$[\mathrm{eV}]$} & - & - & - & 0 & 0 & 0.7810 \\
\hline$\beta_{i j}$ & {$[1 / \AA ̊]$} & - & - & - & 0 & 0 & 1.25 \\
\hline$R^{*}{ }_{i j}$ & {$[\AA \AA]$} & - & - & - & 0 & 0 & 2.369 \\
\hline & [e] & & +4 & & & +2.4 & \\
\hline$Z_{0}$ & [e] & & -2 & & & -1.2 & \\
\hline
\end{tabular}


One of the advantages of the Busker model is the transferability of the potential between a variety of elements - it also has parameters for $\mathrm{U}^{3+}$ and $\mathrm{U}^{5+}$, thereby allowing the effects of off-stoichiometry to be studied (see below). By contrast, the Yamada model is a Bushing-Ida type potential ${ }^{7}$ given by $^{4}$ :

$$
\phi\left(r_{i j}\right)=A_{i j} \exp \left(-r_{i j} / \rho\right)-\frac{c_{i j}}{r_{i j}{ }^{6}}+D_{i j}\left[\exp \left(-2 \beta\left(r_{i j}-r_{i j} *\right)\right)-2 \exp \left(-\beta\left(r_{i j}-r^{*}\right)\right)\right]
$$

The first two terms are the Buckingham potential terms and the last two terms are the Morse terms, which provide a "covalent" component. Due to the assumption of partial covalency, the charges of the ions are given by non-formal values intended to represent the partial charge transfer between ions (see Table 3-1).

To avoid the prohibitive computational expense associated with the calculation of the electrostatic interaction using the Ewald method, the electrostatic interactions are calculated using the direct summation method ${ }^{8}$. The direct summation technique involves truncation of the electrostatic force at a fixed cutoff radius, with charge compensation on the surface of the truncation sphere. The method has been demonstrated to be accurate and successfully applied to a number of materials ${ }^{8,9}$. It is also computationally very efficient, straightforward to implement, and has a computational load that scales linearly with system size.

Table 3-2 summarizes the structural parameters and elastic properties determined using the two potentials. By construction, both give good values for the lattice parameters. The General Utility Lattice Program (GULP) ${ }^{10,11}$ is used to determine the elastic properties at $300 \mathrm{~K}$. GULP uses a static method based on the quasi-harmonic approximation and thus provides slightly lower estimated values for $\mathrm{C}_{11}$ than direct MD simulation, which includes the dynamical motion of the ions. We can see that while the Busker potential reproduces the value of $\mathrm{C}_{12}$ rather well, it overestimates $\mathrm{C}_{11}$ and $\mathrm{C}_{44}$. By contrast, the Yamada potential gives good estimates of $\mathrm{C}_{11}$ and $\mathrm{C}_{44}$, but severely underestimates $\mathrm{C}_{12}$. As a result one overestimates and the other underestimates the bulk modulus $B=\left(C_{11}+2 C_{12}\right) / 3$.

Table 3-2. Lattice parameter and elastic constants at $300 \mathrm{~K}$.

\begin{tabular}{ccccccc} 
& & \multicolumn{2}{c}{ GULP } & \multicolumn{2}{c}{ MD } & \multirow{2}{*}{ Experiment $^{13-17}$} \\
& & Busker & Yamada & Busker & Yamada & \\
\hline a & {$[\AA]$} & 5.481 & 5.482 & 5.479 & 5.481 & 5.478 \\
$\mathrm{C}_{11}$ & {$[\mathrm{GPa}]$} & 526 & 409 & 547 & 418 & $389-396$ \\
$\mathrm{C}_{12}$ & {$[\mathrm{GPa}]$} & 118 & 55.0 & & & $119-121$ \\
$\mathrm{C}_{44}$ & {$[\mathrm{GPa}]$} & 118 & 53.4 & & & $59.7-64.1$ \\
$\mathrm{~B}$ & {$[\mathrm{GPa}]$} & 257 & 174 & & & $209-213$
\end{tabular}

There are numerous other interatomic potentials for $\mathrm{UO}_{2}$ in the literature. Recently, Govers et al. ${ }^{12}$ undertook an extensive comparison of a number of empirical potentials for $\mathrm{UO}_{2}$, including the two used in this study. Their assessment included both rigid-ion and shell models, and they calculated cohesive energy, lattice parameter, elastic constants, dielectric constants, Г-point phonon frequencies, and defect formation energies. While some potentials seemed to give a better physical description than others, their results show that no single potential faithfully reproduces all of the physical properties of $\mathrm{UO}_{2}$. The two potentials 
used in here are thus representative of other $\mathrm{UO}_{2}$ potentials with regards to their materials fidelity.

\subsection{Simulation of Thermal Expansion}

The thermal expansion can be determined in simulation in a way directly analogous to the experimental approach. That is, the material is heated at zero pressure, and the temperature dependence of the lattice parameter is determined.

The actual simulation process consisted of a series of simulations on a relatively small simulation cell (6x6x6 unit cells). At every temperature, the system was allowed to equilibrate at zero pressure for 20,000 MD steps; values for the zero-pressure lattice parameter were then accumulated for 50,000 MD steps. (One single time step corresponds to 0.25 femto-sec; therefore, 50,000 steps are equivalent to 12.5 peco-sec.) Over the range of temperature we have examined (from 0 to $2000 \mathrm{~K}$ ), the uranium dioxide remains in the cubic fluorite structure. The lattice expansion is thus calculated by averaging the expansion in all three directions. The mean lattice expansion and the estimated uncertainty in the value were then determined from the last 30,000 data points.

The temperature dependence of the lattice parameter from the Busker and Yamada potentials are shown in Figure. 3-1. As we expect, the lattice parameter is an approximately linear function of the temperature, which means that the thermal expansion is only weakly temperature dependent. In the same figure, a polynomial fit by Fink to numerous experimental data is shown. ${ }^{13}$ The fits consist of two distinct regions of temperature; below and above 923K. The lattice parameter obtained from the Busker potential shows a maximum deviation of $0.5 \%$ at $1500 \mathrm{~K}$ from the experimental value. The Yamada potential clearly gives a better fit to the experimental results. Linear fits to the Fink's compilation, Busker and Yamada potential give the thermal expansion coefficients of $11.9 \times 10^{-6} \mathrm{~K}^{-1}$, $7.02 \times 10^{-6} \mathrm{~K}^{-1}$ and $9.65 \times 10^{-6} \mathrm{~K}^{-1}$, respectively. These values of thermal expansion are used as inputs to the modified FRAPCON codes, as discussed in Sec. 2. 


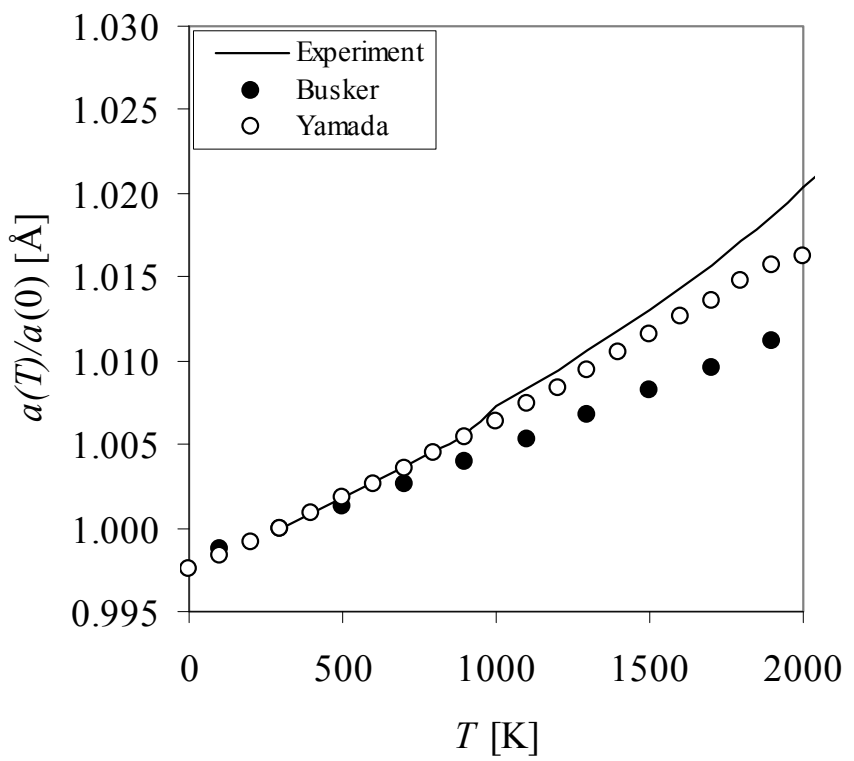

Figure 3-1. Mean lattice parameter of a $6 \times 6 \times 6$ simulation cell at $300 \mathrm{~K}$.

\subsection{Anharmonicity Analysis}

The thermal expansion coefficient is a result of the anharmonicity of the interactions in the material. This is encapsulated in the Grüneisen relation:

$$
\alpha=\frac{\gamma c_{v}}{3 B}
$$

where $c_{v}$ and $B$ are the specific heat and bulk modulus respectively, and $\gamma$ is the Grüneisen parameter, which measures the dependence of the phonon frequencies on system volume, and is thus the system's anharmonicity ${ }^{14}$. If the interatomic interactions were purely harmonic, then the Grüneisen parameter would be zero and there would be no thermal expansion. Thus the higher thermal expansion of the Yamada model can be interpreted as a result of higher anharmonicity in the potential compared to the Busker potential.

The lattice thermal conductivity of any material is finite because of the anharmonicity of the interatomic interactions that couple the harmonic phonons to each other. Anharmonicity results in phonon scattering events, with the Umklapp processes producing a dissipation mechanism for energy transport. ${ }^{14,15}$

An estimate of the lattice thermal conductivity in terms of the Grüneisen parameter was first given by Leibfried and Schloemann ${ }^{16}$ and refined by Klemens ${ }^{17}$,

$$
\kappa \sim \frac{24}{10} \frac{\sqrt{4}}{\gamma^{2}}\left(\frac{k_{B}}{h}\right)^{3} M v \frac{\theta^{3}}{T} .
$$


Here $\mathrm{k}_{\mathrm{B}}$ is the Boltzmann constant, $h$ is the Planck constant, $v$ and $M$ are the volume and the mass per atom respectively. The only two materials constants that enter into Eq. (3-4) are $\theta$, the Debye temperature, and $\gamma$ the frequency-averaged Grüneisen parameter. From this relation, it is clear that the thermal conductivity decreases with increasing anharmonicity.

Through their dependences on the Grüneisen parameter, we can use Eqs. (3-3) and (3-4) to derive a simple relationship between the thermal conductivity and thermal expansion, in terms of the Debye temperature, the bulk modulus and the specific heat:

$$
\alpha^{2} \kappa=\chi \frac{\theta^{3} c_{v}^{2}}{B} \text {. }
$$

The constant $x$ subsumes all of the non-materials constants in Eq. (3-3) and (3-4). In classical simulations at temperatures above the Debye temperature $\left(370 \mathrm{~K}\right.$ for $\left.\mathrm{UO}_{2}\right)$, the specific heat is essentially equal to the Dulong-Petit value of $3 k_{B}$. Also the Debye temperatures for the two potentials are assumed to be the same. Hence we find:

$$
\kappa=\frac{\chi^{\prime}}{\alpha^{2} B},
$$

where $x^{\prime}=x \theta^{3} c_{v}^{2}$.

Using the values of $K$ and $B$ determined above for the two potentials, we then predict:

$$
\begin{gathered}
\alpha_{\text {Busker }} \sim 8 \times 10^{-5} X^{\prime} \\
\alpha_{\text {Yamada }} \sim 10 \times 10^{-5} X^{\prime}
\end{gathered}
$$

This simple analysis would thus predict that the Yamada potential would give a $\sim 25 \%$ larger thermal conductivity than the Busker potential.

Using the experimental values of $B$ and $\alpha$, Eq. 3-6 gives $\kappa_{\text {Expt }} \sim 3.4 \times 10^{-2} X^{\prime}$, which is considerably smaller than the predictions for the Busker and Yamada potentials. Thus, based on this very naïve analysis, we expect the direct simulations with the two potentials to give thermal conductivities for the Busker and Yamada potentials that are 3.0 and 2.4 times larger respectively than the experimental values; as we shall see in the next sub-section, these estimates are quite accurate.

\subsection{Thermal Conductivity of Single Crystal}

In principle, the simulation of the thermal conductivity is also closely analogous to a simple experimental approach. We follow the method of Jund and Julien ${ }^{1824}$, modified by Schelling and Phillpot. ${ }^{19,20}$ Figure 3-2 shows a side view of the simulation cell. It has a small cross section in the $x-y$ plane (at least $4 x 4$ unit cells for uranium dioxide) and is relatively much longer in the $z$ direction (typically 48 unit cells). Periodic boundary conditions are imposed in all three spatial directions; thus the material does not have any surfaces. To create a thermal current along the length of the simulation, heat is added in slab of material of width $\delta=2$ unit cells located at $z=0$, and heat is removed from an identical slab at $z=L_{z} / 2$. This is actually 


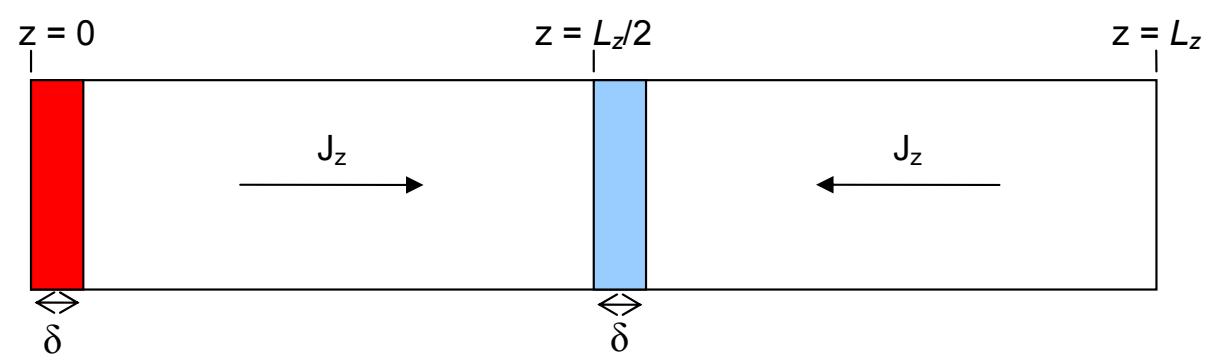

Fig. 3-2. Schematic of three-dimensional periodic simulation cell for direct simulation of $\tilde{\kappa}$ Simulation cell has length, $L_{z}$. There is slab of thickness $\delta$ at $z$ $=0$, into which energy $\Delta \varepsilon$ is added at each MD step. Likewise, in slab at $z=$ $L_{z} / 2$, energy $\Delta \varepsilon$ is removed at each step. The resulting thermal currents are $J_{z}$.
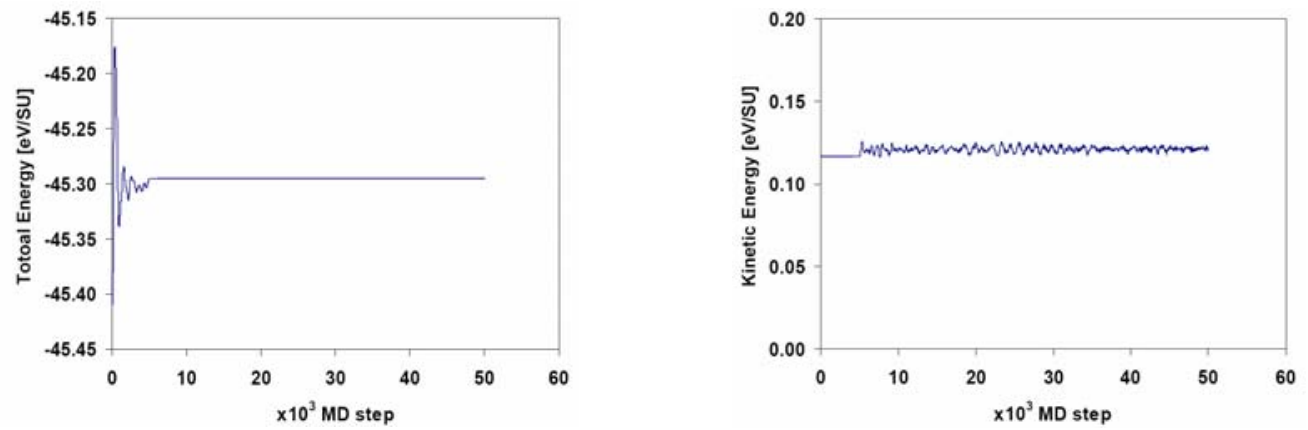

Fig. 3-3. Total energy (left) and kinetic energy (right) per stoichiometric unit of $\mathrm{UO}_{2}$.from the simulation of $4 \times 4 \times 48$ unit cell system with heat source and heat sink. It shows good energy conservation and small fluctuations of kinetic energy, a measure of the temperature fluctuations intrinsic to these microcanonical ensemble simulations.

performed by rescaling the particle velocities at each MD time step in two thin slabs. By removing kinetic energy $\Delta \varepsilon$ at from the 'cold' slab at each MD time step and adding it to the 'hot' slab, the total energy of the system is conserved. Due to the periodic boundary conditions in $\mathrm{z}$, this heating and cooling sets up two identical thermal currents in the systems and produces temperature gradients.

In general, the thermal conductivity, $\kappa$, is determined by Fourier's Law:

$$
J_{z}=-\kappa \cdot \frac{d T}{d z} .
$$

In terms of the variables of the simulation itself, the thermal conductivity is given by: 


$$
\kappa=-\frac{\Delta \varepsilon}{2 A \cdot d t} \cdot \frac{d T}{d z},
$$

where $d t$ is the MD time step, $\Delta \varepsilon$ is the heat added and removed at each time step, $A$ is the cross sectional area, and $d T / d z$ is the temperature gradient set up in the system.

Although the simulation approach for determining the thermal conductivity is quite simple, actual implementation is a little more complex. First, a large simulation cell is required, necessitating the use of a parallel simulation code. Moreover, it takes an extremely long simulation time (in excess of 1 million MD time steps) for the temperature gradient in the simulation cell to reach steady state. The reliable determination of the temperature gradient itself takes a further $\sim 1$ million steps. All of the thermal-conductivity simulations are performed at fixed volume with the lattice corresponding to zero pressure for the simulation temperature, as determined from the previously described thermal-expansion simulations. A typical run to obtain the thermal conductivity of one composition at one temperature takes 35 days on 12 CPUs.

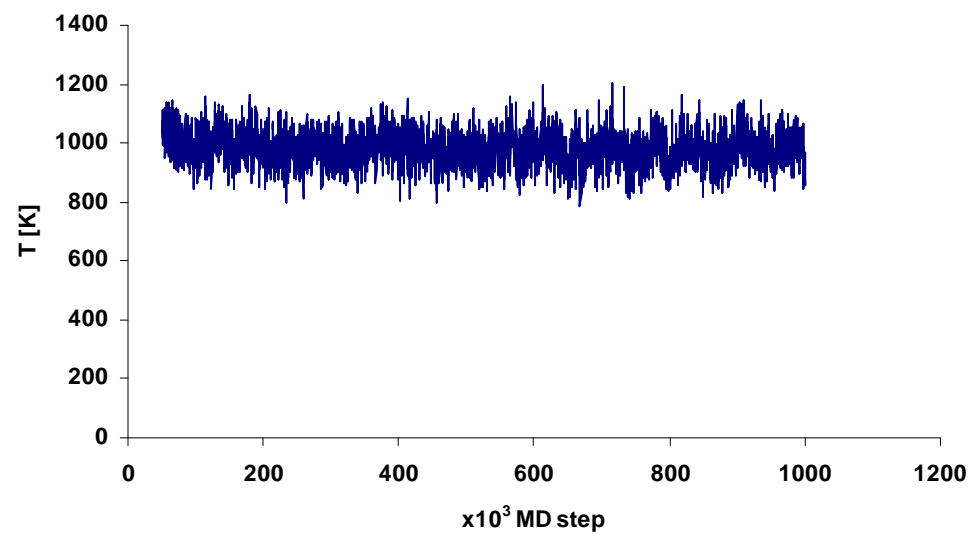

Fiq. 3-4. Temperature at $z=-L z / 4$ of a $4 \times 4 \times 48$ simulation cell at $1000 \mathrm{~K}$.

Before the actual thermal conductivity calculation is done, several tests were performed to check the code. After the implementation of the Busker and Yamada potentials in the parallel code, the behavior of the energies has been analyzed to make sure the code is running properly. Figure 3-3 shows the kinetic, and total energies of the system using Yamada potential. The unit of energy is in eV per stoichiometric unit of $\mathrm{UO}_{2}$. The simulation was run for $50000 \mathrm{MD}$ steps, corresponding to $12.5 \mathrm{psec}$. Initially the simulation was started with constant temperature constant volume for the equilibration. At step 5000, the heat source and heat sink were turned on but there was no energy input/output. This part of the simulation was performed in the microcanonical ensemble, i.e., at constant volume and constant energy. Therefore the average temperature (i.e. kinetic energy) in the system should fluctuate around a fixed value and the energy should stay constant. As Figure. 3-3 shows the energies are behaving as expected.

With a finite input energy $(0.001 \mathrm{eV})$, behavior of the temperature between the heat source and sink are monitored. Figure 3-4 shows the temperature at the location equal distance from heat source and heat sink. The temperature is calculated from the kinetic energy averaged over the slice of simulation cell with a unit cell thick and over every $200 \mathrm{MD}$ steps. The simulation was run for 1 million MD steps at mean temperature of $1000 \mathrm{~K}$. The temperature is fluctuating around $1000 \mathrm{~K}$ and indicates no sign of drift. 
Figure 3-5 shows the temperature gradient obtained in a thermal conductivity simulation using the Busker potential with a $4 \times 4 \times 48$ simulation cell at $300 \mathrm{~K}$. The heat source and sink are located at the $z=0$ and $13 \mathrm{~nm}$. The simulation was run for 1.5 million MD steps, equivalent to $375 \mathrm{psec}$. From the linear regions (approximately $8 \mathrm{~nm}$ ) of this temperature profile, the data is fitted to a straight line and the temperature gradient is calculated. In this case, the temperature gradients are 3.36 and $3.57 \mathrm{~K} / \mathrm{nm}$. Using the relation given in the previous section, the thermal conductivity has turned out to be 19.4 $\mathrm{W} / \mathrm{mK}$. (Figure. 3-5) This value is almost twice as high as experimental value. ${ }^{21}$ At $1000 \mathrm{~K}$, the value turned out to be $8.55 \mathrm{~W} / \mathrm{mK}$.

It is known that the thermal conductivity depends weakly on the cross sectional area. $^{20}$ Therefore the cross-section area is set to the $4 \times 4$ unit cells, which is the smallest size that can be simulated with the cutoff values used in these potentials of $R_{c}=1.98 \mathrm{a}$, where a

is

the

lattice

parameter.

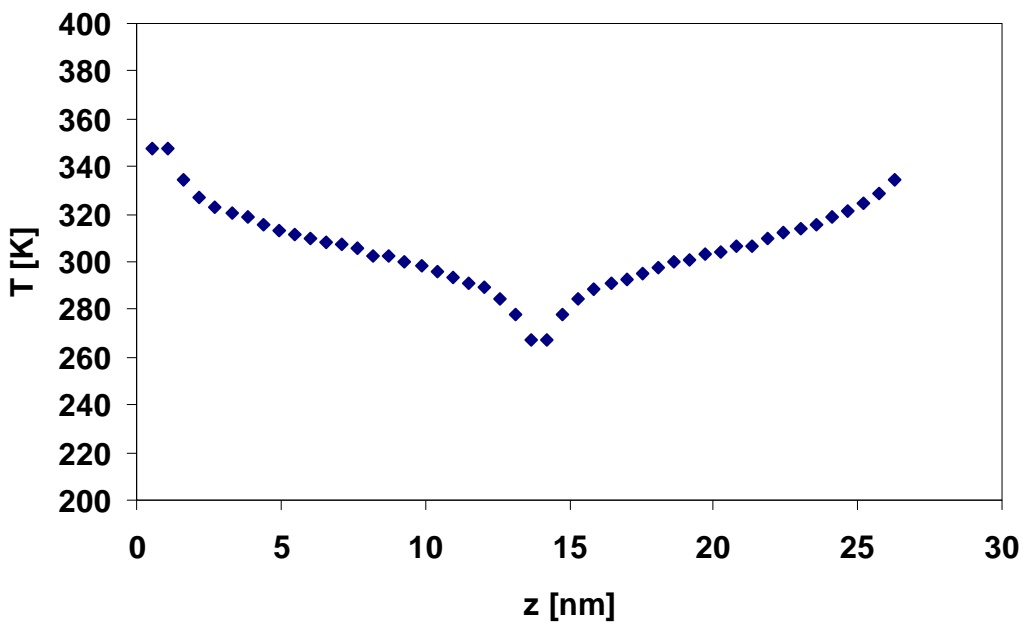

Fig. 3-5. Temperature profile of a $4 \times 4 \times 48$ simulation cell at $300 \mathrm{~K}$.

The thermal conductivity of single crystal $\mathrm{UO}_{2}$ between $300 \mathrm{~K}$ and $2000 \mathrm{~K}$ is determined as a function of system length. The results for the two potentials are shown in Figure. 3-6 as 1/K vs. $1 / L_{z}$ plots. The results for the two potentials are similar at all temperatures and system sizes. 

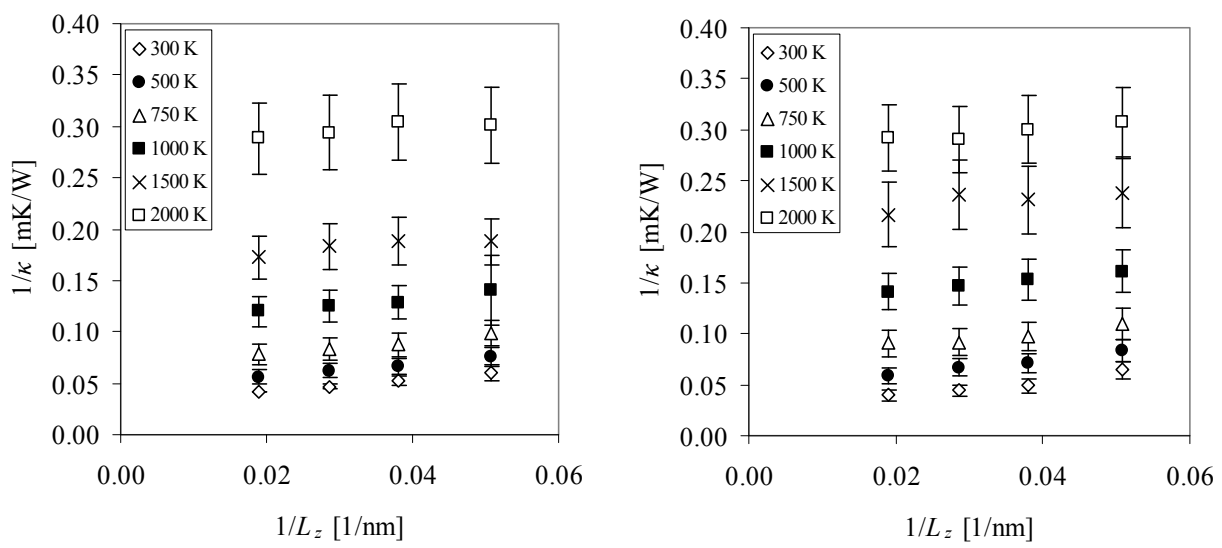

Figure 3-6. Size dependence of thermal conductivity for both the Busker (left) and Yamada (right) potentials.

The thermal conductivity for infinite system size is determined by the linear fits to the data in Figure 3-6. The relation between the thermal conductivity and system size is given by a simple kinetic theory of phonon gas:

$$
\frac{1}{\kappa}=\frac{a^{3}}{N_{c} c_{v} v}\left(\frac{1}{l_{\infty}}+\frac{4}{L_{z}}\right)
$$

$\mathrm{N}_{\mathrm{c}}$ is the number of atoms in the unit cell. $\mathrm{V}$ is the mean sound velocity in the solid. These infinite size limit thermal conductivities, which are the best estimates of the intrinsic thermal conductivity of $\mathrm{UO}_{2}$ described by these potentials, are shown for the two potentials in Figure 3-7(a) as a function of temperature. Although the error bars overlap, it does appear that the Yamada potential gives a somewhat higher estimated thermal conductivity at low $\mathrm{T}$ than does the Busker potential, but has a somewhat stronger temperature dependence. This result is reasonably consistent with the only small difference in thermal conductivity predicted by the simple anharmonic analysis performed above

As Figure 3-7(a) indicates, both potentials give significantly higher thermal conductivities at low temperatures than the experimental values. Moreover, the factor of 3-4 difference is in at least qualitative agreement with the factor of $2.5-3.5$ predicted in our simple analysis. It thus attributes the majority of the difference between the experimental and simulation values as arising from the differences in the bulk modulus, which measures the harmonic properties of the system, and the thermal expansion, which measures the anharmonic properties of the system. The thermal conductivity corrected according to the anharmonicity analysis, Figure. 3-7(b), is in much better agreement with the experimental values. This demonstrates the efficacy of the process of performing an anharmonic correction analysis

The anharmonic Umklapp processes lead to the temperature dependence of the thermal conductivity. Debye showed that $\alpha \sim \mathrm{T}^{-\alpha}$, with $\alpha \sim 1-2^{29}$. Figure 3-7 (c) is a log-log plot of the data in Figure. 3-7 (a). In each case, the thermal conductivity shows power-law behavior with temperature. The experimental results are fitted by $\alpha_{\text {Expt }} \sim 0.79$, while the simulations yield $\alpha_{\text {Yamada }} \sim 1.14$ and $\alpha_{\text {Busker }} \sim 1.30$ respectively, which are consistent with the Debye analysis. 

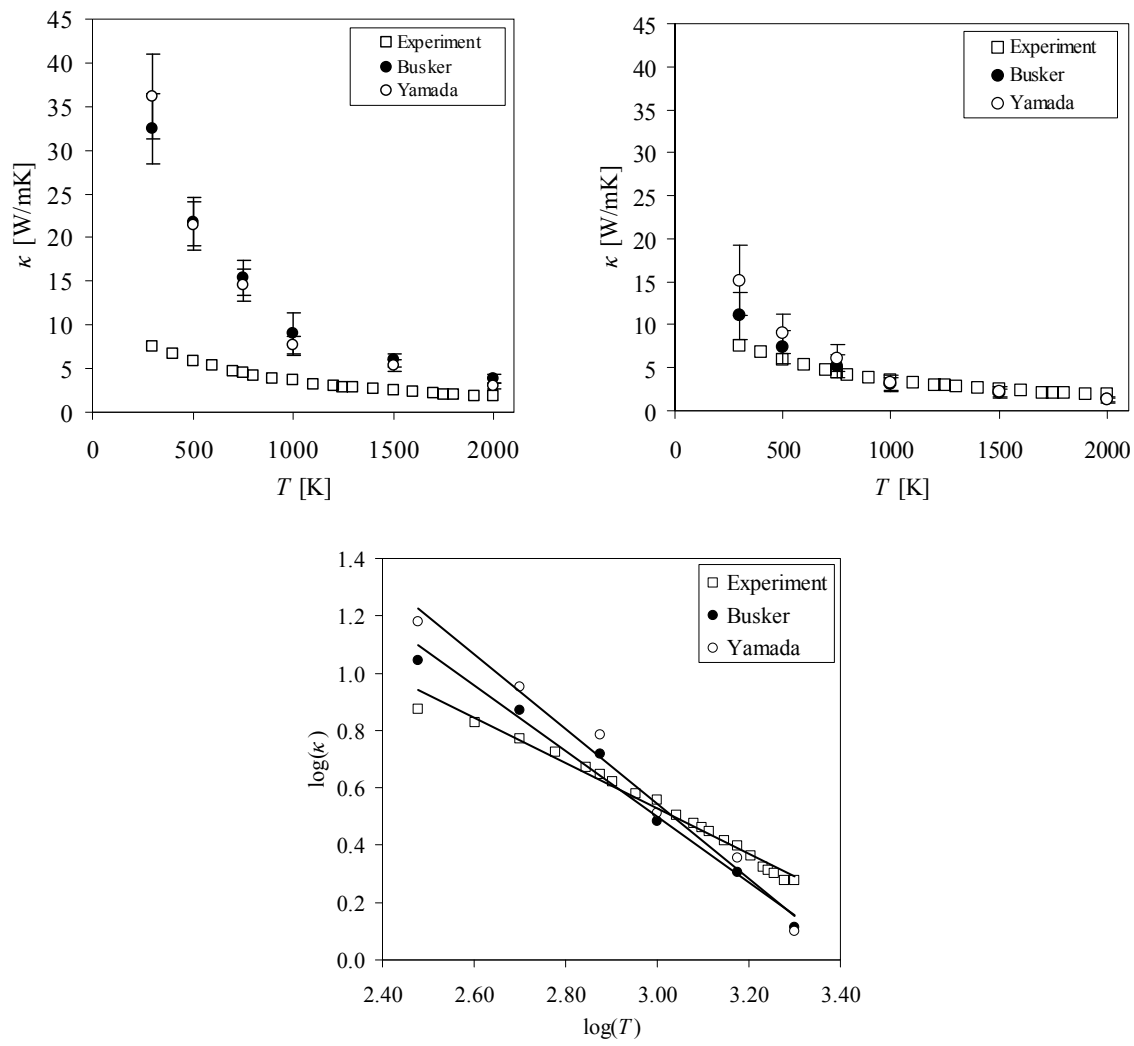

Figure 3-7. (a) Thermal conductivity of $\mathrm{UO}_{2}$ from the compilation of experimental data by Fink and simulations using the Busker and Yamada potentials. (b) When corrected according to the anharmonicity analysis, the predicted thermal conductivity is in much better agreement with the experimental values. (c) A log-log plot of the same data shows the expected powerlaw temperature dependence.

\subsection{Thermal Conductivity of Polycrystalline $\mathrm{UO}_{2}$}

Grain boundaries offer a significant obstacle to the transport of heat in phonon conductors. In this section, we determine the thermal conductivity of a model fine-grained polycrystal of $\mathrm{UO}_{2}$, from which we make predictions for the grain-size dependence of its thermal conductivity.

\subsubsection{Structure of Model Polycrystal}

Experimental grain sizes of the range of tens or hundred of microns are not accessible to MD simulation, since each grain would contain $\sim 10^{13}-10^{16}$ ions. We simulate 
considerably smaller systems with grain sizes from $3.8-6.5 \mathrm{~nm}$; these small sizes maximize the area of the grain boundary in the system, thereby amplifying the interfacial effects.

The polycrystalline structures used in the simulations consisted of 24 hexagonal columnar grains. When constructing the polycrystalline structure, identical close-packed hexagons are arranged to form a completely periodic structure. Each hexagon is filled with single-crystal $\mathrm{UO}_{2}$ oriented with [001] along the columnar direction. The in-plane orientations are chosen in such a way that the grain boundaries (GBs) between the grains are high energy tilt GBs, which ensures that the microstructure is stable against coarsening during the simulation. Because of the way each grain is constructed, initially there are always a small number of atoms in the GBs which are extremely close to each other. To address this issue, if any two ions are closer than $1.5 \AA$ (66\% of the nearest neighbor distance between $\cup^{4+}$ and $\mathrm{O}^{2-}, 2.29 \AA$ ), one of the atoms is removed. This removal of atoms is carried out with care to ensure the charge neutrality of the entire system. Once the structure is created, it is quenched at $0 \mathrm{~K}$ to equilibrate all of the atom positions and to eliminate any in-plane stress on the system. It was found that no ions have anomalously high energies, indicating that the bonding in the system is physically reasonable. The system was then annealed with a constant-pressure, constant-temperature simulation at $2000 \mathrm{~K}$ and slowly relaxed to $0 \mathrm{~K}$ to ensure that the structure is equilibrated. Figure 3-8 shows the final relaxed polycrystalline $\mathrm{UO}_{2}$ structure for a grain size of $3.8 \mathrm{~nm}$.

The columnar microstructure used here allows the simulation cell to be thin along the columnar direction. The cutoff to the potentials is $10.4 \AA$, which would allow the thickness to be as small as 4 unit cells: in our simulations, we use 5 unit cells thick so as minimize any effects of the system size in that direction.

Since all the grains are equiaxial and equal size, it is easy to calculate the grain boundary area and volume fraction. For a grain size of $3.8 \mathrm{~nm}$, the area of the GB is 439.10 $\mathrm{nm}^{2}$. If we assume a unit cell for the thickness of the grain boundary, the volume which the grain boundary region occupies is approximately $30 \%$ of the entire volume in both models. The structural disorder at the GBs led to a total volume expansion of $5.5 \%$ and $5.3 \%$ for Busker and Yamada potentials respectively. The corresponding GB energies are $2.73 \mathrm{~J} / \mathrm{m}^{2}$ and $1.89 \mathrm{~J} / \mathrm{m}^{2}$, which are consistent with the GB energies of other ceramic materials. ${ }^{22,23}$

After the preparation of the equilibrated structure, the thermal expansion of this polycrystalline $\mathrm{UO}_{2}$ was determined at $300 \mathrm{~K}$. The values obtained were $7.57 \times 10^{-6} \mathrm{~K}^{-1}$ with Busker potential and $8.92 \times 10^{-6} \mathrm{~K}^{-1}$ for Yamada potential, which are almost indistinguishable from the corresponding bulk single crystal values of $7.50 \times 10^{-6} \mathrm{~K}^{-1}$ and $8.83 \times 10^{-6} \mathrm{~K}^{-1}$. 


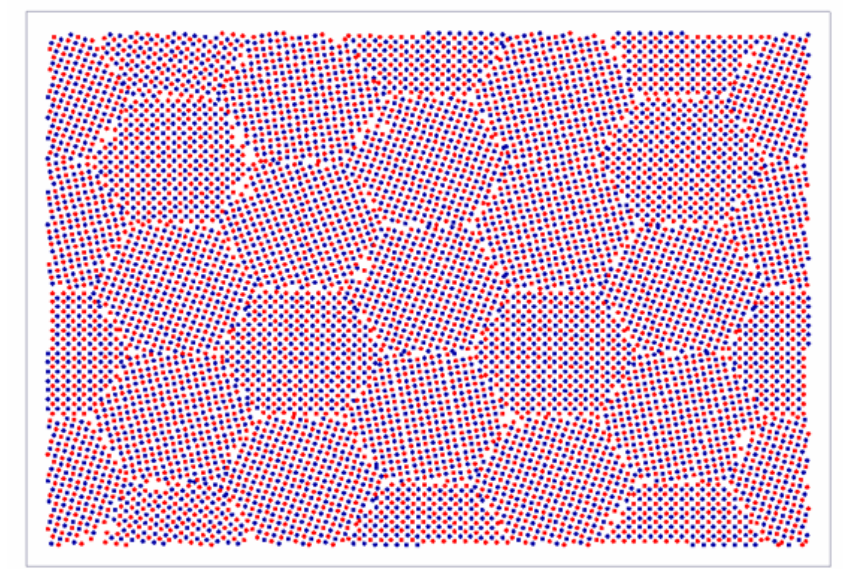

Figure 3-8. Polycrystalline structure of $\mathrm{UO}_{2}$. Red is oxygen and blue is uranium. The grain size is $3.8 \mathrm{~nm}$ and the structure consists of 24 columnar grains.

\subsubsection{Thermal Conductivity}

The thermal conductivity of polycrystalline $\mathrm{UO}_{2}$ is calculated using the direct method described above. The thermal conductivity of the polycrystal has been scaled according to the anharmonic analysis. In this step, we have assumed that the thermal expansion and bulk modulus are essentially microstructure independent. We are aware that this is not strictly true. However, within the approximation made in separating the contributions in the polycrystal thermal conductivity from that of grain interior and GB, the accuracy may not be sufficient to consider more detailed analysis. Fig. 3-9(a) shows the temperature dependence of $\kappa$ for a polycrystal with a grain size of $3.8 \mathrm{~nm}$. These calculated thermal conductivities are considerably lower than those from the perfect crystal calculations, attesting to the significant resistance of GB to the flow of heat through the system. Unlike single crystalline $\cup_{2}$, finite size effects are small in polycrystals because the grain boundary scattering dominates over the phonon-phonon scattering. Elsewhere, we have characterized the effect of system size on polycrystalline $\mathrm{MgO}^{24}$, and shown that the system size dependence is weak. Since $\mathrm{UO}_{2}$ has significantly lower thermal conductivity than $\mathrm{MgO}$, the effect is expected to be even less important.

The thermal conductivity for the polycrystal described is considerably higher for the Busker potential than for the Yamada potential. Since the GB energy is a measure of the structural disorder at the interfaces, we expect that the higher the energy associated with the GBs, the higher the interfacial thermal resistance, and the lower the thermal conductivity of the polycrystal. We recall that the GB energies obtained from the Busker and Yamada are $2.73 \mathrm{~J} / \mathrm{m}^{2}$ and $1.89 \mathrm{~J} / \mathrm{m}^{2}$, which appears to be inconsistent with this argument. However, the Busker potential is a full charge model, while the Yamada potential is a partial charge model, resulting in cohesive energies of $-104.482 \mathrm{eV} / \mathrm{UO}_{2}$ and $-45.54 \mathrm{eV} / \mathrm{UO}_{2}$ respectively. Thus, when normalized to the bulk cohesive energies, the GB energies are $0.0026 \AA^{-2}$ and 0.0016 $\AA^{-2}$ for Yamada and Busker, respectively. That is, when described by the Busker potential, the GBs actually offer less of an obstacle to heat transport than for the Yamada potential, which is consistent with the higher thermal conductivity for the Busker potential than for the Yamada potential. 
The ensemble-averaged interfacial (Kapitza) resistance of the grain boundaries in the polycrystal can be extracted from the thermal conductivity using a simple model. There are several models proposed, including those of Nan and Birringer ${ }^{25}$, Yang et al. ${ }^{26}$, and Amrit ${ }^{27}$. For this analysis, we adopt the model by Yang et al. ${ }^{26}$ in which the polycrystal is assumed to consist of perfect crystal grains, with conductivities of the perfect crystal, separated by GBs, all of which have the same thermal properties. The interfacial conductance, $G_{K}$, is then given by:

$$
G_{K}=\frac{1}{d} \frac{\kappa_{0} \kappa}{\kappa_{0}-\kappa},
$$

where $d$ is the grain size, $\kappa_{0}$ is the single crystal thermal conductivity, and $\kappa$ is the thermal conductivity of polycrystalline $\mathrm{UO}_{2}$.

The resulting values of $G_{K}$ are given in Figure 3-9(b). Both potentials show moderate increase of interfacial conductance with temperature. This temperature increase is consistent with simulation of other interfacial systems ${ }^{28}$ and, more importantly, with trends in experimental data for various systems ${ }^{29}$. The physical origin of the increase in conductance with temperature can be understood in terms of the properties of the grain boundaries. As the temperature increases, the anharmonicity of the interactions among the atoms is probed more strongly. While in the perfect crystal, the resulting scattering lowers the thermal conductivity, this anharmonic scattering more strongly couples modes across the interfaces, leading to better interfacial thermal transport.

The interfacial conductance can be recast in terms of the Kapitza length, $I_{K}=\kappa_{0} / G_{K}$, where $\kappa_{0}$ is thermal conductivity of infinite size single crystal $U_{2}$. The Kapitza length is the thickness of perfect crystal that would offer the same thermal resistance as the interface; thus a long Kapitza length corresponds to a high interfacial resistance. As shown in Figure 39(c), the Kapitza length decreases strongly with increasing temperature; this is a result of the decrease in the thermal conductivity and the increase in the interfacial conductance with temperature.

We note that for both potentials, the Kapitza length is significantly larger than the grain diameter particularly in the low temperature region. This is an indication that the thermal transport in our model system is dominated by the GBs. It also suggests that the fundamental assumption of separable and grain-size bulk and interfacial thermal properties used for the analysis may be violated at these small grain sizes.

We have also investigated the effects of grain size on thermal conductivity, for grain sizes up to $6.5 \mathrm{~nm}$ grains. The inset to Figure 3-10 shows the expected increase in thermal conductivity with increasing grain size, the result of the decrease in the relative volume of grain boundaries in the system. Our data for polycrystals simulated with the Yamada potential are fit to the model of Yang et al., thereby allowing the thermal conductivity for large grain sizes to be estimated. Taking the bulk single crystal conductivity of $15.2 \mathrm{~W} / \mathrm{mK}$ at 300 $\mathrm{K}$, the fit gives the Kapitza conductance of $0.15 \mathrm{GW} / \mathrm{m}^{2} \mathrm{~K}$, which is close to the value previously determined for the $3.8 \mathrm{~nm}$ polycrystal (see Figure 3-10). 

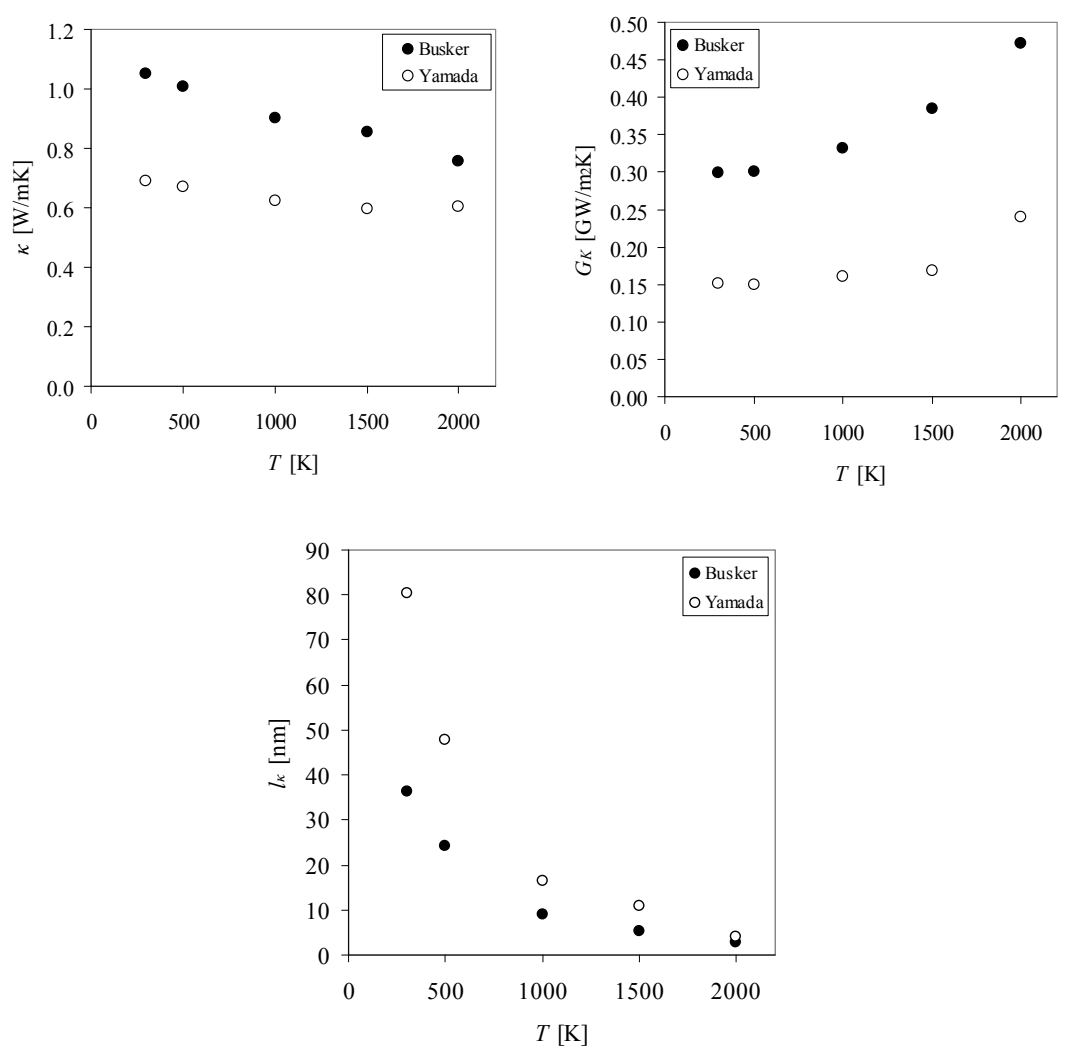

Figure 3-9. (a) Thermal conductivity of $3.8 \mathrm{~nm}$ grain polycrystalline $\mathrm{UO}_{2}$ from our simulations. (b) Thermal conductance grain boundaries from $3.8 \mathrm{~nm}$ grain polycrystalline $\mathrm{UO}_{2}$ simulations. (c) Kapitza length from $3.8 \mathrm{~nm}$ grain polycrystalline $\mathrm{UO}_{2}$ simulations. 


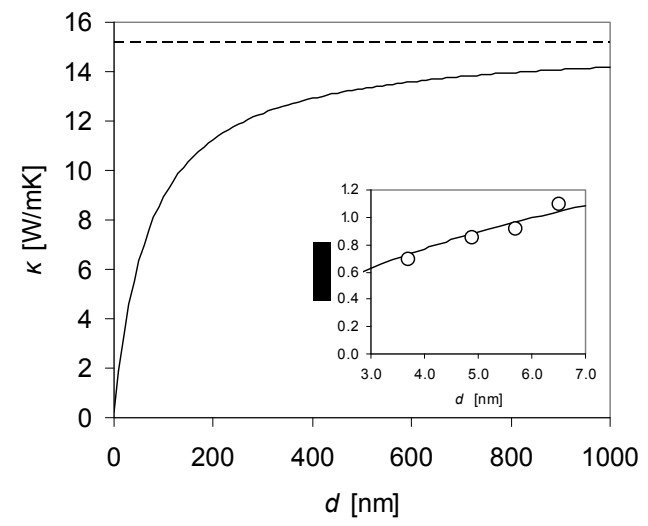

Figure 3-10. Grain size dependence of the thermal conductivity of polycrystalline $\mathrm{UO}_{2}$ using Yamada's potential. The solid line is the fit of the model by Yang et al. ${ }^{34}$. Inset is the same plot for nano-meter scale indicating the fit with our data.

\subsubsection{Discussion}

Although finite-size effects are rather significant in single crystal $\mathrm{UO}_{2}$, they seem to be less of an issue in the simulation of the polycrystals, particularly at these small grain sizes, presumably because the phonon mean free path is limited by the grain boundaries, rather than by anharmonic phonon interactions. The analysis of the polycrystal to obtain the interfacial conductance is not unambiguous, since the calculated Kaptiza lengths are larger than the grain sizes. However, the fact that the calculated thermal conductivities of polycrystals of different grains sizes, albeit over the narrow range of $3.8-6.5 \mathrm{~nm}$, can be fit to the Yang et al. model, strongly suggest that its use is not unreasonable. Moreover, since the same analysis is used throughout, the trends of interfacial conductance with temperature is reasonable.

Neither potential can quantitatively match the experimental thermal conductivity. Based on the analysis of Govers et al. ${ }^{12}$, the two potentials used in the study are of a similar level of materials fidelity as others in the literature. The simple relationship, Eq. (3-6), relating the elastic properties, thermal expansion and elastic constants, suggests that a potential with correct elastic properties and thermal expansion, should well reproduce the thermal-transport properties. A truly general purpose potential would also need to well reproduce the point defect properties. Govers et al. ${ }^{12}$ showed that none of the twenty-one potentials they examined could satisfactorily reproduce the formation and migration energies. There is thus considerable need for potentials which better describe $\mathrm{UO}_{2}$.

\subsection{Effects of Off-Stoichiometry on Thermal Conductivity}


Just as grain boundaries do, point defects also reduce the thermal conductivity of solids. It is well known that the $\mathrm{UO}_{2}$ is prone to oxidation and easily form the hyperstoichiometric phases. Formation of $\mathrm{UO}_{2-\mathrm{x}}$ is also possible although it is limited to very high temperature or in the case of accident condition. Effect of the deviation from the stoichiometry to the thermal transport was investigated.

\subsubsection{Thermal Transport in Disordered Solid}

In crystalline solids, the thermal transport is limited either by phonon-phonon scattering above the Debye temperature, or phonon-defect (point defects, dislocations, grain boundaries, surfaces) scattering below the Debye temperature. In a highly disordered solid, the thermal transport mechanism is fundamentally different from that in crystalline solids. Thermal conductivity of a crystalline solid is a function of $T^{3}$ below the Debye temperature ${ }^{14}$. In a disordered solid, the temperature dependence is, in general,

$$
\kappa \propto T^{3-n}
$$

where $n$ is an integer. In the case of amorphous material, $\mathrm{n}$ is close to unity and the temperature dependence is therefore $T^{2}{ }^{30,31}$. Specific heat in this case is almost linear in temperature $^{15}$. These dependence of thermal conductivity and specific heat can be explained in terms of scattering of acoustic phonons from two level states ${ }^{31}$. Above the Debye temperature, $k$ increases slowly with $T$ and eventually becomes almost temperature independent. This temperature independent regime can be described in terms of a bounded phonon mean free path ${ }^{32,33}$. Slack ${ }^{33}$ calculated the thermal conductivity of the Debye solid, assuming that the mean free path of the phonon is half of its wavelength for each mode. With these assumptions, he defined the minimum thermal conductivity of glasses. Cahill and $\mathrm{Pohl}^{34}$ made the same assumption and gave the minimum thermal conductivity in terms of the Debye integral. Although these models give reasonable description of highly disordered solid, the physical basis of thermal transport using phonon gas model is questionable. The main issue is that the phonon mean free path in these materials is so short (order of interatomic distance) that the idea of phonons itself seem inappropriate.

Allen et al. ${ }^{35,36}$ took a different approach to this problem, and developed a model for the thermal conductivity of amorphous materials at high temperatures. Their model is based on the harmonic approximation to analyze the vibrational modes. They demonstrated that the majority of the thermal energy is transferred through diffusive modes termed "diffusons". In amorphous $\mathrm{Si}$, roughly $93 \%$ of the vibrational modes correspond to the diffuson modes. Other modes in the higher frequency bands are called "locons" due to the fact that they are truly localized. In the other extreme of the phonon spectrum, the low frequency modes are similar to phonons, having well defined wave vectors and polarization vectors. They are called "propagons" and consist only about $\sim 3 \%$ of the entire vibrational modes. Although the propagons are very efficient in transferring thermal energy, their contribution to thermal conductivity is minimal because of their small population. Within this approach, the temperature dependence of thermal conductivity mostly comes from the temperature dependence of specific heat. This in turn agrees with the temperature independent behavior at high temperature. This approach developed by Allen et al. is useful not only in amorphous solid, but also in heavily doped materials ${ }^{9}$.

\subsubsection{Thermal Transport in $\mathrm{UO}_{2}$ with Point Defects}

In $\mathrm{UO}_{2}$, the predominant point defects are anti-Frenkel pairs ${ }^{13,37,38,39}$. The defect formation energies of anti-Frenkel, Frenkel and Schottky defects are approximately $4 \mathrm{eV}, 10 \mathrm{eV}$, and 6 
eV. Although the Schottky trio formation energy is higher than anti-Frenkel pair, they play important role at high temperature ${ }^{13,38}$.

There is also a recent MD simulation study performed by Yamasaki et al. ${ }^{40}$ on the thermal conductivity of $\mathrm{UO}_{2+x}$. They employed the Green-Kubo method to calculate the thermal conductivity by an equilibrium MD simulation. They have shown that their predicted thermal conductivity gives good agreement with experimentally observed conductivity data. Their data show a decrease of conductivity with increasing concentration of defects. Although their data show the trend observed in the experiments and expected from the phonon-defect scattering, their data do not show any error bars which are at least comparable to the difference between their conductivity data.

\subsubsection{Preparation of the Structures}

In order to perform MD simulations with oxygen defects, interatomic potentials must be modified. The modifications are simply to include $\mathrm{U}^{3+}$ and $\mathrm{U}^{+5}$ in the interatomic potential. This is necessary to retain the charge neutrality of the whole system. The potential parameters are given in Table 3-3. These parameters were provided by Prof. Robin Grimes of Imperial College London, as part of our ongoing collaboration

Table 3-3. Potential parameters for the short-range $\mathrm{U}^{5+}-\mathrm{O}^{2-}$ and $\mathrm{U}^{3+}-\mathrm{O}^{2-}$ interactions.

\begin{tabular}{|cc|cc|}
\hline & & $\mathrm{U}^{5+}-\mathrm{O}^{2-}$ & $\mathrm{U}^{3+}-\mathrm{O}^{2-}$ \\
\hline$A_{i j}$ & {$[\mathrm{eV}]$} & 2386.42 & 1165.65 \\
$\rho_{i j}$ & {$[\AA]$} & 0.3411 & 0.3786 \\
$C_{i j}$ & {$\left[\mathrm{eV} \cdot \AA^{6}\right]$} & 0 & 0 \\
\hline
\end{tabular}

The defect structures are prepared in the following fashion. First the single crystalline $\mathrm{UO}_{2}$ structure is prepared in the desired size. Then a number of excess oxygen atoms corresponding to desired concentration are inserted in randomly selected octahedral sites. In addition, randomly selected $\mathrm{U}^{4+}$ atoms are replaced with $\mathrm{U}^{5+}$ atoms such that overall charge neutrality is maintained. Once the structure is created, an MD simulation is performed at $3000 \mathrm{~K}$ for $10 \mathrm{psec}$ to ensure the complete equilibration. After equilibration, the system is slowly cooled to $0 \mathrm{~K}$ in a step wise fashion. A similar procedure is performed to create oxygen deficient structures. From the single crystal $\mathrm{UO}_{2}$, an appropriate number of the randomly selected oxygen atoms are removed to create a desired concentration of oxygen vacancies. At same time, randomly selected $\mathrm{U}^{4+}$ atoms are replaced with $\mathrm{U}^{3+}$ atoms for charge neutrality. After the structure is created, the structural equilibration process is performed.

These off-stoichiometric structures are characterized by the pair distribution function analysis. Figures 3-11 and 3-12 show the pair distribution functions between $\mathrm{U}^{5+}$ and $\mathrm{O}^{2-}$, and $\mathrm{U}^{3+}$ and $\mathrm{O}^{2-}$. In both cases, there is no difference between the pair distribution functions of $\mathrm{U}^{3+}, \mathrm{U}^{4+}, \mathrm{U}^{5+}$ and $\mathrm{O}^{2-}$ since all the cations are at identical sites. Before the relaxation, all the peaks corresponding to oxygen ions at tetrahedral sites in FCC are quite prominent. In the $\mathrm{UO}_{2+\mathrm{x}}$ structure, there are small peaks just by the tetrahedral oxygen peaks arising from the interstitial atoms. After annealing and quenching to $0 \mathrm{~K}$, the difference in the pdf between $\mathrm{U}^{4+}-\mathrm{O}^{2-}$ and $\mathrm{U}^{5+}-\mathrm{O}^{2-}$ in $\mathrm{UO}_{2+x}$ can be seen. The peaks are broadened and the height decreased. Furthermore, the first peak of the pdf of $\mathrm{U}^{5+}-\mathrm{O}^{2-}$ is shifted inward compared to the $\mathrm{U}^{4+}-\mathrm{O}^{2-}$ by $\sim 0.2 \AA$. This shift occurs because of the stronger electrostatic attraction of $\mathrm{U}^{5+}-\mathrm{O}^{2-}$ than $\mathrm{U}^{4+}-\mathrm{O}^{2-}$. Other peaks are also shifted, but the differences are so small that they cannot 
be seen in the figure. In the anion deficient material, the pdf is again broadened after the equilibration, but the peak positions did not move at all.

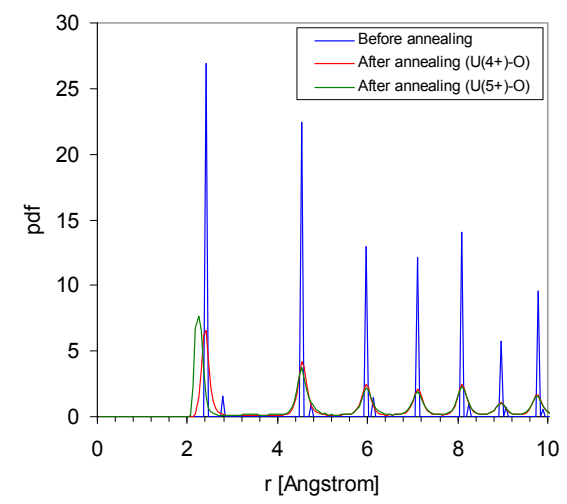

Figure 3-11. Pair distribution function between $U-O$ in $U_{1.25}$. Blue curve is the $U-O$ pdf for both $\mathrm{U}^{4+}-\mathrm{O}$ and $\mathrm{U}^{5+}-\mathrm{O}$. There is no difference before relaxation.

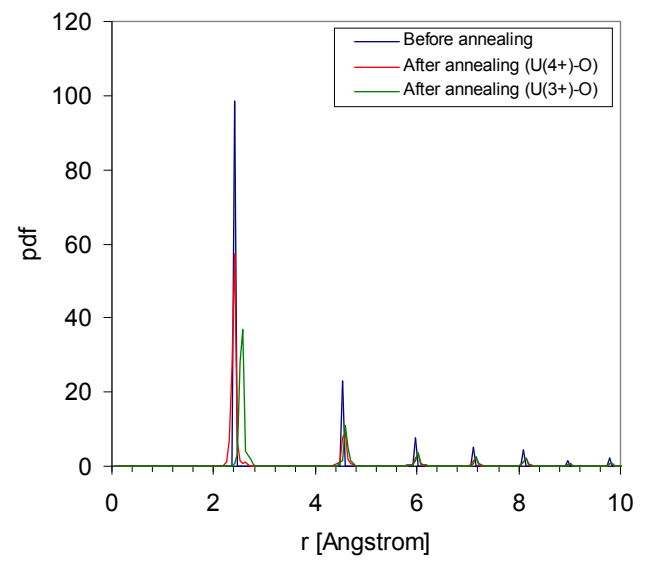

Figure 3-12. Pair distribution function between $\mathrm{U}-\mathrm{O}$ in $\mathrm{UO}_{1.80}$. Blue curve is the $\mathrm{U}-\mathrm{O}$ pdf for both $\mathrm{U}^{4+}-\mathrm{O}$ and $\mathrm{U}^{3+}-\mathrm{O}$.

Figure 3-13 shows the composition dependence of the lattice parameter of $\mathrm{UO}_{2+\mathrm{x}}$ at $0 \mathrm{~K}$ after relaxation (normalized by the lattice parameter of stoichiometric $\mathrm{UO}_{2}$ ). The lattice parameter of $\mathrm{UO}_{2+x}$ decreases with increasing concentration of oxygen interstitials due to the increased electrostatic attractions by the $\mathrm{U}^{5+}$ atoms introduced in the system for the charge neutrality requirement. Figure 3-14 shows the corresponding plot of the normalized lattice parameter 
of for $\mathrm{UO}_{2-\mathrm{x}}$. Here the lattice parameter increases with increasing concentration of oxygen vacancies due to the reduced electrostatic interaction by the introduction of $\mathrm{U}^{3+}$ in the system. Another notable difference from $\cup_{2+x}$ is that the linearity of the data. This is not surprising considering the much lower range of concentration of the defects in $\mathrm{UO}_{2-\mathrm{x}}$ than in $\mathrm{UO}_{2+\mathrm{x}}$.

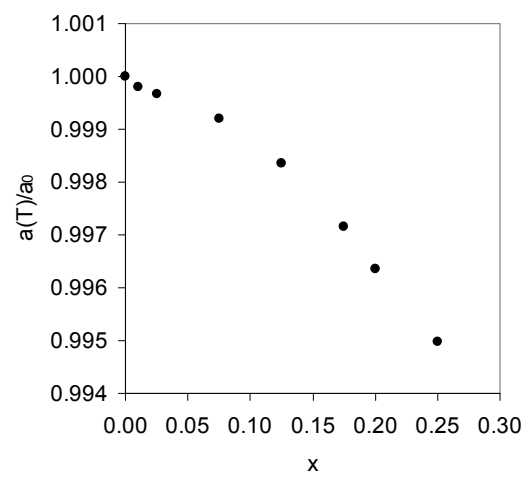

Figure 3-13. Chemical expansion of $\cup_{2+x}$. The lattice parameters are determined for $0 \mathrm{~K}$ after relaxation.

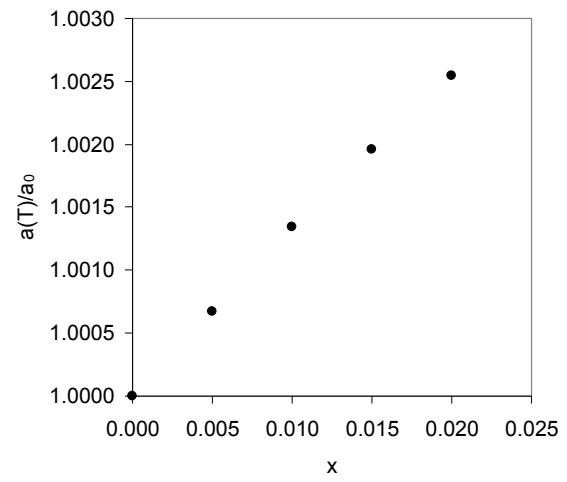

Figure 3-14. Chemical expansion of $\mathrm{UO}_{2-x}$. The lattice parameters are determined for $0 \mathrm{~K}$ after relaxation.

\subsubsection{Thermal Expansions of $\mathrm{UO}_{2 \pm x}$}

Figures 3-15 and 3-16 show the thermal expansion of $\mathrm{UO}_{2+x}$ and $\mathrm{UO}_{2-\mathrm{x}}$ respectively. For $\mathrm{UO}_{2+\mathrm{x}}$, the thermal expansion actually decreases with increasing concentrations for the same reason that the chemical expansion decreases (the increased strength of electrostatic interactions). No concentration effect is apparent in $\mathrm{UO}_{2-x}$ due to the only small range of offstoichiometry. 


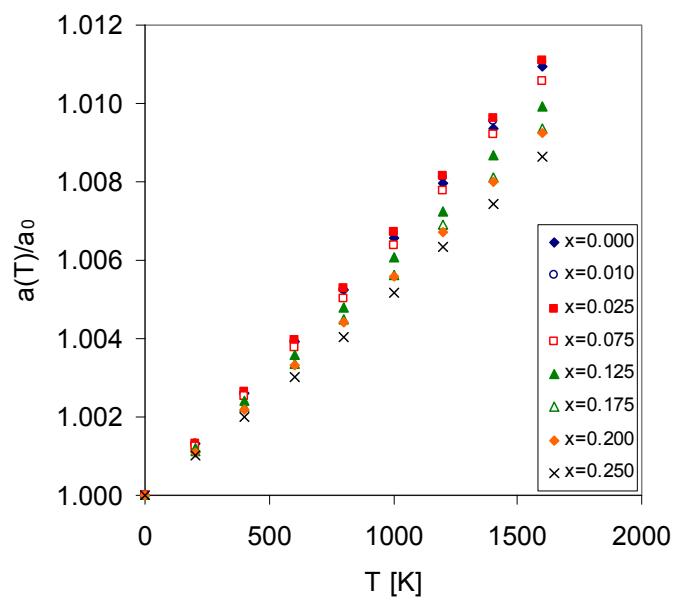

Figure 3-15. Thermal expansion of $\cup_{2+x}$. Lattice parameters are normalized by the $0 \mathrm{~K}$ lattice parameter for the corresponding composition.

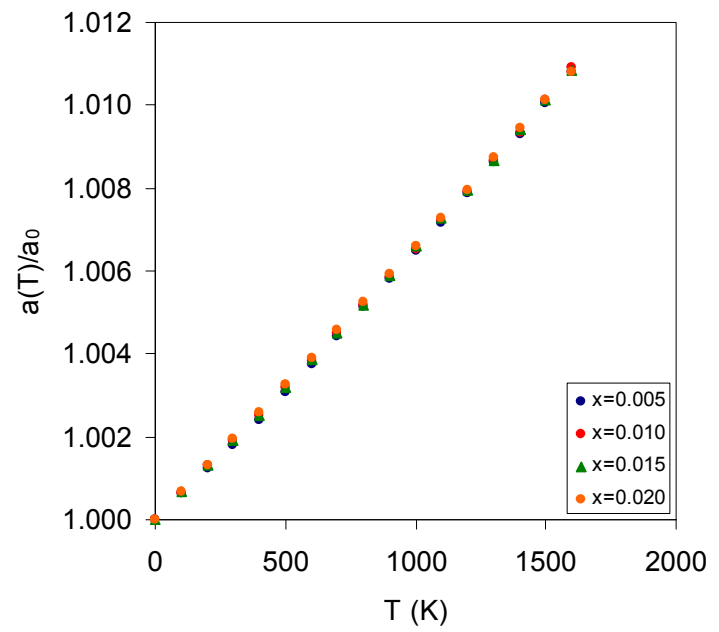

Figure 3-16. Thermal expansion of $\cup_{2-x}$. Lattice parameters are normalized by the $0 \mathrm{~K}$ lattice parameter for the corresponding composition.

\subsubsection{Thermal conductivity of $\mathrm{UO}_{2 \pm x}$}

Using the technique described above, $\cup_{2+x}$ structures of $4 \times 4$ unit cells cross sections are prepared with the simulation cell length $L_{z}=48,64,80$, and 96 unit cells. The range of stoichiometry investigated was between $x=0$ and 0.25 . This range is chosen based on the thermodynamic stability range of $\mathrm{UO}_{2+x}$ between 800 and $1600 \mathrm{~K}$, and is beyond the range of 
off-stoichiometry in any of the previous experimental and simulation studies. The thermal conductivity simulations were run at 800 and $1600 \mathrm{~K}$, which correspond to the surface and centerline temperature of the fuel pellet in operating condition. A series of runs were performed over the range of stoichiometry and simulation cell length. The results are shown Figure 3-17. The data points are obtained from the linear fit to the thermal conductivity data from all the simulation cell length. For the low concentration of defects $(x \leq 0.075)$, the data fits quite well. However, at the higher concentrations of defects $(x \geq 0.0125)$, the data points do not follow the linear fit as well as the lower concentration. At the highest defect concentration $(x=0.250)$, the fit is out of the trend. Similar fit is done on the data set at 1600 $\mathrm{K}$ also.

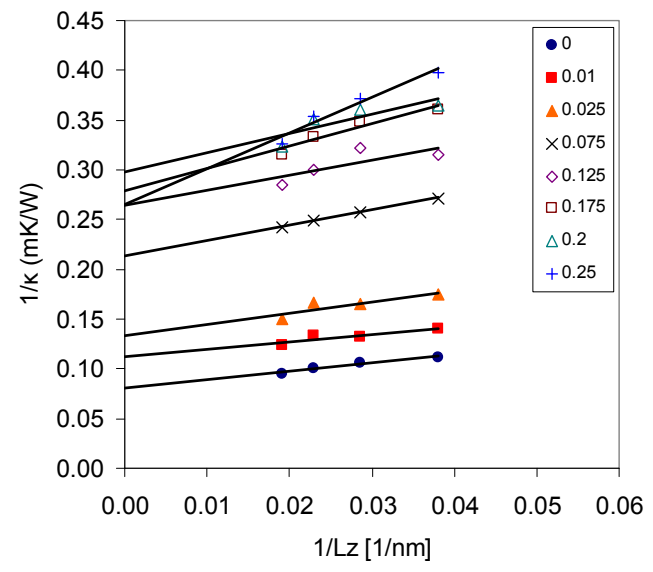

Figure 3-17. Thermal conductivity of $\mathrm{UO}_{2+x}$ at $800 \mathrm{~K}$ as a function of system length.

From the linear fit to the data, the thermal conductivity of an infinitely large system is obtained by the extrapolation to $1 / L_{z}$. These results, which are our best estimate of the true thermal conductivity, are shown in Figure. 3-18. The data show smooth monotonic decrease of the conductivity with increasing concentration of oxygen interstitials at $800 \mathrm{~K}$. The conductivity value eventually plateaus at $\sim 3.8 \mathrm{~W} / \mathrm{m} \cdot \mathrm{K}$ for $\mathrm{x} \geq 0.125$. This value compares well with the minimum thermal conductivities of $2 \mathrm{~W} / \mathrm{m} \cdot \mathrm{K}$ of yttria-stabilized zirconia (YSZ) and lanthanum-doped $\mathrm{CaF}_{2}$ measured by Cahill et al. ${ }^{41}$ and $\sim 3 \mathrm{~W} / \mathrm{m} \cdot \mathrm{K}$ determined by the MD simulations of $\mathrm{YSZ}$ by Schelling et al. ${ }^{9}$ At $1600 \mathrm{~K}$, the data is rather noisy as expected by the significant phonon-phonon scattering.

Within the context of the Callaway's model, ${ }^{42}$ phonon-defect scattering rate is proportional to the defect concentration originally given by Klemens ${ }^{42,43}$ :

$$
\tau^{-1} \propto n_{i}
$$

where $n_{i}$ is the concentration of defect species $i$. Since the thermal conductivity is proportional to the relaxation time, this relation implies that the thermal conductivity is inversely proportional to the defect concentration. Figure 3-19 shows the same MD simulation data on a log-log scale. The data appears to be fit well with a straight line for 800 $\mathrm{K}$ data indicating that the power law relation of $\kappa \sim x^{-\alpha}$ is reasonable. However, the data from 
$1600 \mathrm{~K}$ simulations are quite noisy. From the fit exponent $\alpha$ is determined to be $\sim 0.32$ at 800 $\mathrm{K}$ and $\sim 0.15$ at $1600 \mathrm{~K}$.

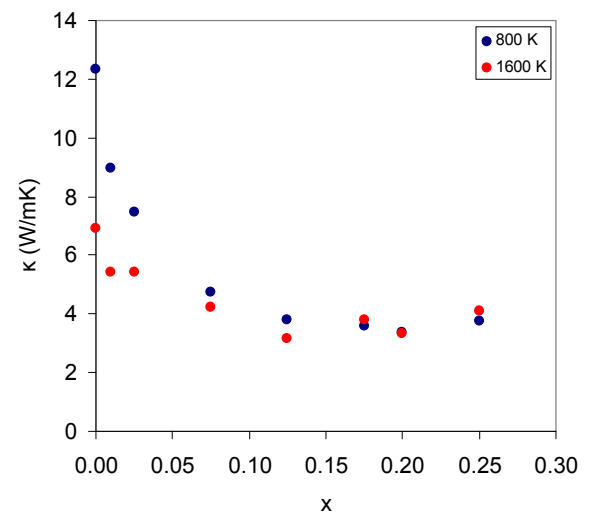

Figure 3-18. Thermal conductivity of $\mathrm{UO}_{2+x}$ at 800 and $1600 \mathrm{~K}$.

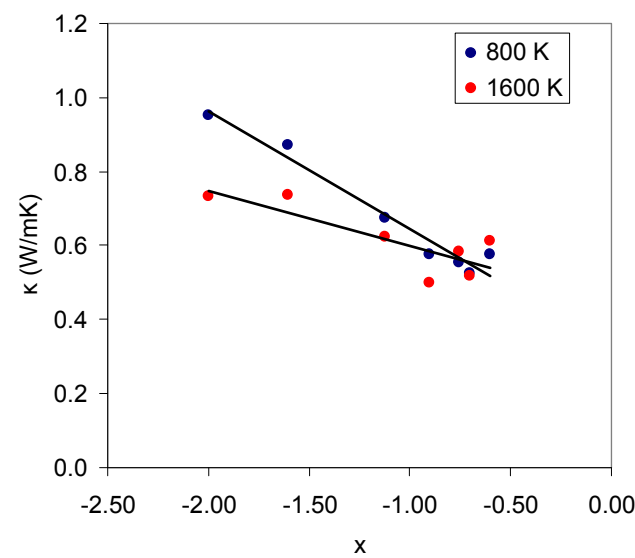

Figure 3-19. Log-log plot of the thermal conductivity as a function of defect concentration at 800 and $1600 \mathrm{~K}$.

\subsection{Radiation Damage Simulation}

$\mathrm{UO}_{2}$ is under constant irradiation by neutrons and by $\alpha, \beta$, and $\mathrm{y}$ radiations. The high energy radiation by a particles or the recoil of the fission products are known to cause structural damage to the crystal and change the properties of the materials. The structural change will be particularly detrimental to the thermal transport properties since heat is carried by the atomic vibrations of the lattice. The work focused on validating the every piece of the 
subroutines that are essential for the radiation damage simulation; namely, ZBL potential, variable times step, and thermostat routines.

\subsubsection{Code Development}

The idea of a radiation damage simulation is to give an excess kinetic energy to an atom in the system, and let it collide with the atoms in its path and study the evolution of the cascade produced. In order to perform this type of simulations, some new capabilities had to be implemented into the molecular-dynamics simulation code we use. These capabilities are the ZBL potential, a variable time step, and thermostat around the structure.

When atoms collide with very high energy, they get too close to each other. The conventional empirical interatomic potentials for MD simulation are not suitable for such a very short-range interactions. A solution to this problem is to use the universal interatomic potential by Ziegler, Biersack, and Littmark (ZBL). ${ }^{44}$ This phenomenological model prevents the Coulomb catastrophe, and prevents atoms from collapsing upon collision. The ZBL potential is given by,

$$
\begin{aligned}
& V_{Z B L}(r)=\frac{q_{1} q_{2}}{r} \cdot \varphi(r / a), \\
& \text { where if } \mathrm{x}=\mathrm{r} / \mathrm{a}, \\
& \varphi(x)=0.1818 e^{-3.2 x}+0.5099 e^{-0.9423 x}+0.2802 e^{-0.4028 x}+0.02817 e^{-0.2016 x} \\
& a=0.8854 a_{0} /\left(Z_{1}^{0.23}+Z_{2}{ }^{0.23}\right)
\end{aligned}
$$

Here, $q_{1}$ and $q_{2}$, and $Z_{1}$ and $Z_{2}$ are the charges and atomic number of the two interacting atoms. $a_{0}$ is the Bohr radius, $r$ is the distance between the two atoms. In our MD code, this potential is smoothly connected to the Busker's or Yamada's potentials through exponential of a $5^{\text {th }}$ order polynomial:

$$
V_{\text {Spline }}(r)=\exp \left(f_{0}+f_{1} r+f_{2} r^{2}+f_{3} r^{3}+f_{4} r^{4}+f_{5} r^{5}\right)
$$

where $f_{i}$ are the fitting parameters. Figure 3-20 shows the U-O interaction potential using Busker model as an example. U-U and O-O also have their own spline and ZBL potentials. The potential energy surface of the short range interaction is divided in 3 sections according to the radial distance:

$$
V_{S R}(r)=\left\{\begin{array}{cc}
V_{\text {Grimes } / \text { Yamada }}(r) & r_{S R}>r \geq r_{S P} \\
V_{\text {Spline }}(r) & r_{S P}>r \geq r_{Z B L} . \\
V_{Z B L}(r) & r_{Z B L}>r
\end{array}\right.
$$

$r_{S R}, r_{S P}$, and $r_{Z B L}$ are the cutoff radii of the short-range interaction, spline, and ZBL potential. 


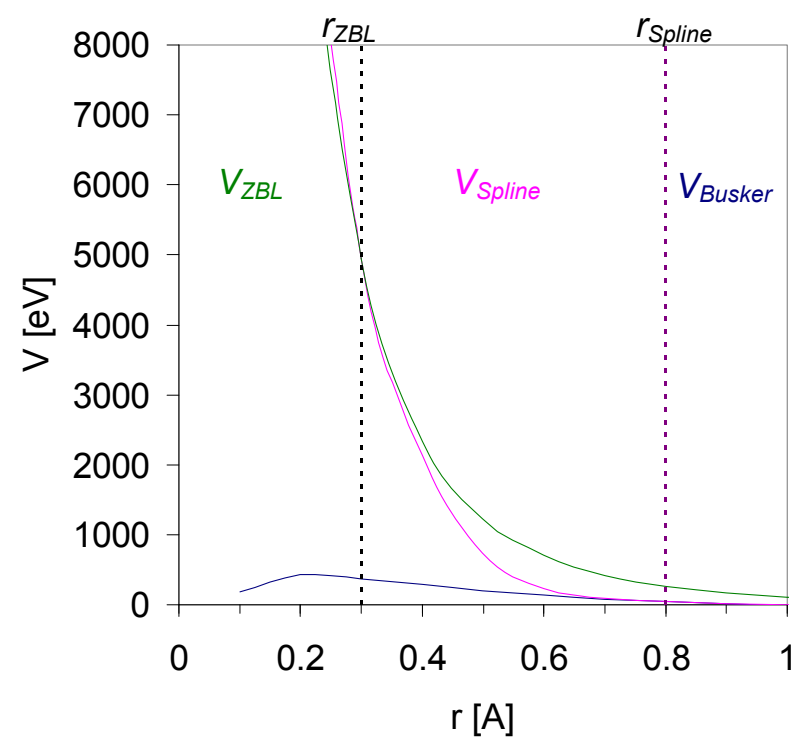

Figure 3-20. U-O interaction energy contributions from ZBL (green), spline (pink) and Grimes' Buckingham potentials. The potentials are smoothly connected at the connection points shown by the dotted lines.

When atoms come very close to each other, the forces on them become very high. If the time step is kept in the conventional time scale, those atoms will fly apart in a single time step in an unrealistic manner. If the time step is kept extremely short to find realistic trajectory, the simulation becomes very inefficient and takes an extremely long time to complete. A time step that can dynamically vary resolves these issues. In our MD simulation code, first the accelerations of all the atoms are checked at certain time interval. According to the acceleration, a trial time step is calculated. Using the trial time step, the next position of the atom with the highest acceleration is predicted with one whole step and two small time step. By comparing the results from these steps, we can obtain the error in the calculation. If this error is below user defined tolerance level, the time step is accepted. If not, the same step is repeated taking the half step time size as the new trial time step. The process continues until the error reaches below the user defined error tolerance. Variable time step improves the efficiency significantly if the error tolerance is chosen appropriately.

Finally the velocity rescaling thermostat is applied around the simulation cell. Thickness of the thermostat region is set to half of the unit cell. All the other atoms move naturally according to the equation of motion. One thing to investigate in the future is the effect of thermostat on the system temperature and the cascade created by the radiation damage.

\subsubsection{Simulation}

The first radiation damage simulation in single crystal $\mathrm{UO}_{2}$ is undertaken using $20 \times 20 \times 20$ unit cells cubic system with 96000 atoms. An atom near the center of the simulation is usually chosen to be the primary knock-on atom (PKA). A kinetic energy of $1 \mathrm{keV}$ is imparted to the PKA, with initial velocities along $\langle 100\rangle,<110\rangle$, and $<111\rangle$ directions. Three simulations 
were performed in each family of directions to assess the statistical variation in the trajectories. The excess heat generated during the simulation was removed by the thermostat atoms surrounding the simulation cell. The thermostat was kept at $300 \mathrm{~K}$ throughout the simulation

Figures 3-21 and 3-22 show the evolution of the number of $O$ and $U$ vacancy produced by $U$ and O PKAs. In general, Yamada's model shows significantly larger number of defects than Busker's model. This is not surprising considering that the ionic charges of $U$ and $\mathrm{O}$ ions are much smaller than those of Busker's: suppressing the long range electrostatic interactions. In Busker's model, the number of $O$ vacancies does not depend on the U PKA directions but Yamada's does. The numbers of $U$ vacancies produced in both models are so low that there is not enough statistics to draw anything conclusive.
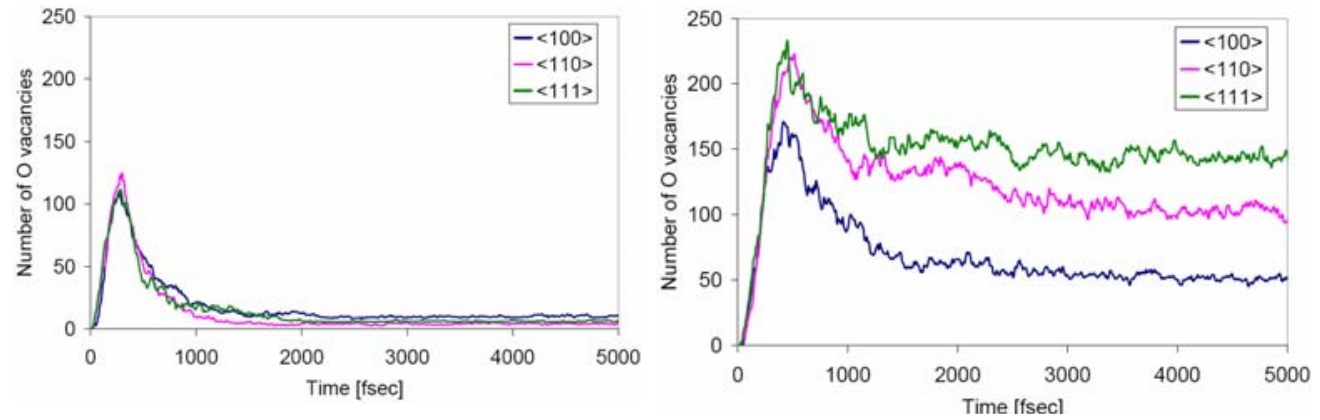

Figure 3-21. Comparison of the number of oxygen vacancies produced by $1 \mathrm{keV}$ PKA from Busker's (left) and Yamada's (right) model. Yamada's model showed significant difference in the anisotropic production of oxygen vacancies, but Busker's model did not.
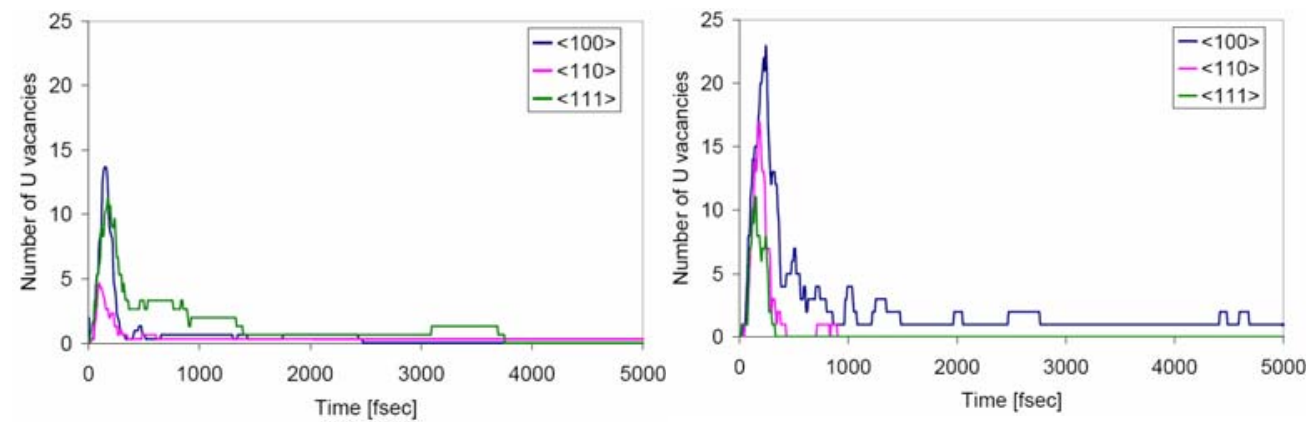

Figure 3-22. Comparison of the number of uranium vacancies produced by $1 \mathrm{keV}$ PKA from Busker's (left) and Yamada's (right) model. Both model show very small numbers of vacancies.

There is also a significant difference in the structure of the defect cascade from the two models. Figure 3-23 shows the final defect structure after $9 \mathrm{psec}$. Busker model leaves very few defects after 1 or $2 \mathrm{psec}$, and the defect is sparse and isolated. Yamada model, on the other hand, generates clusters of defects. Experimentally no amorphization is observed in irradiated $\mathrm{UO}_{2}{ }^{45}$. From the result of $1 \mathrm{keV}$ PKA result, it is not clear that the Yamada's model will not produce amorphous structure of $\mathrm{UO}_{2}$. 

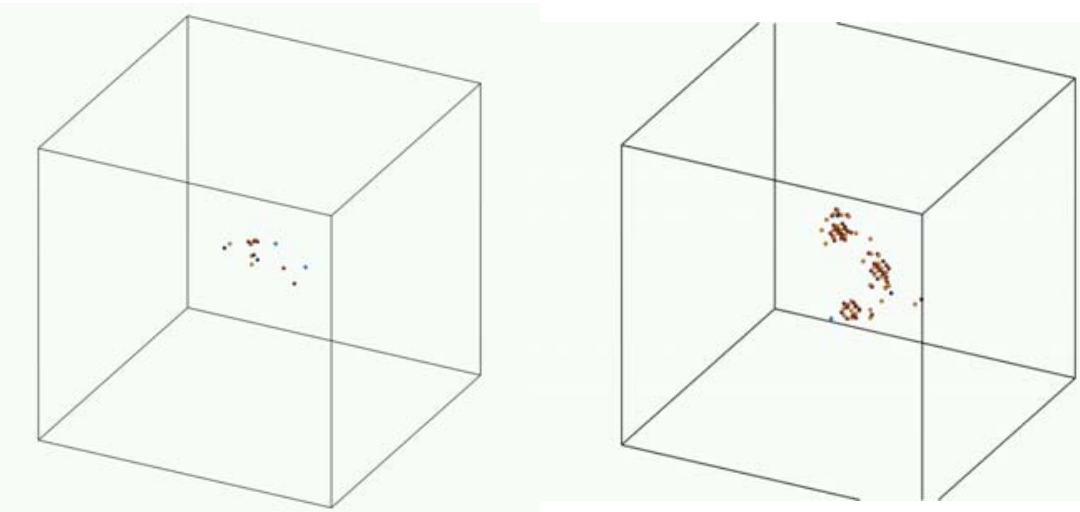

Figure. 3-23. Comparison of the defect structures produced by $1 \mathrm{keV}$ PKA in [100] from Busker (left) and Yamada's (right) model. Light and dark blue are the uranium interstitials and vacancies, respectively. Light and dark orange are the oxygen interstitials and vacancies. The Busker model produced isolated defects but Yamada's showed clusters of defects.

In order to better understand the origin of the differences in defect behavior in the radiation damage simulations, we calculated defect formation energy using our MD code. For this calculation, the same $20 \times 20 \times 20$ unit cells structure from the radiation damage simulation was used. In each simulation, we generated a Frenkel pair (FP) with the atoms are separated by as far as possible ( $10 \mathrm{~nm}$ apart) within the simulation cell along the body diagonal. The structure is then relaxed at $300 \mathrm{~K}$ and slowly cooled to $0 \mathrm{~K}$ and quenched.

Table 3-4 shows the result compared to the experimental value. Calculation of the Frenkel pair (FP) formation energies using our MD simulation code indicates significant difference in the uranium FP formation energies between the two models although the O FP formations energies are almost identical.

We can understand the smaller number of point defects produced in the radiation damage simulation with the Busker potential as arising from the much larger $U$ Frenkel defect formation energy. Notably, however, while the O Frenkel defect formation energies for the two potentials are rather similar, they are both twice as large as the experimental values. We are currently working on identifying a potential with more realistic defect formation energies.

TABLE 3-4. Comparison of the Frenkel pair formation energies by Busker and Yamada models. All values are given in eV. U FP formation energies differ by a factor of 2 between the two models although the O FP formation energies are virtually identical.

\begin{tabular}{|c|c|c||c|}
\hline & Experiment & Busker & Yamada \\
\hline \hline U Frenkel & 9.5 & 24.8 & 12.0 \\
\hline O Frenkel & 3.5 & 7.1 & 7.4 \\
\hline
\end{tabular}


The objective of this task is to use systematic atomic-level simulations to elucidate the fundamental thermo-mechanical properties of $\mathrm{UO}_{2}$ and $\mathrm{MOX}$, and to provide simulation results to the modified FRAPCON code.

The focus of this year's work has been three-fold (i) to investigate the effects of microstructures on the thermal transport properties of $\mathrm{UO}_{2}$ described by two representative interatomic potentials; (ii) to characterize the effects of off-stoichiometry on thermal transport; and (iii) to validate our simulation approach to the simulation of irradiation damage in $\cup_{2}$.

\subsubsection{ZBL potential}

We have performed a simple simulation to show the ZBL potential at work. This simulation was performed on a $10 \times 10 \times 10$ unit cells single crystal $\mathrm{UO}_{2}$. A Uranium PKA was given kinetic energy of $1 \mathrm{keV}$ in [100] direction and the system average potential and kinetic energies were monitored. Figure 3-24 is the results of this toy model simulation. Since this is a single crystal $U_{2}$, the $U$ PKA continued to collide with other $U$ in its path. This process appears as peaks and cusps in the potential and kinetic energy plots. The entire simulation was performed with constant energy constant volume ensemble, and therefore the total energy remained at $-15.0905 \mathrm{eV} /$ atom for the $50 \mathrm{fs}$. Comparing between with and without the ZBL potential, the effect is rather clear. The ZBL potential acts like a hard steep potential causing the potential energy curve to change very rapidly upon collision. 

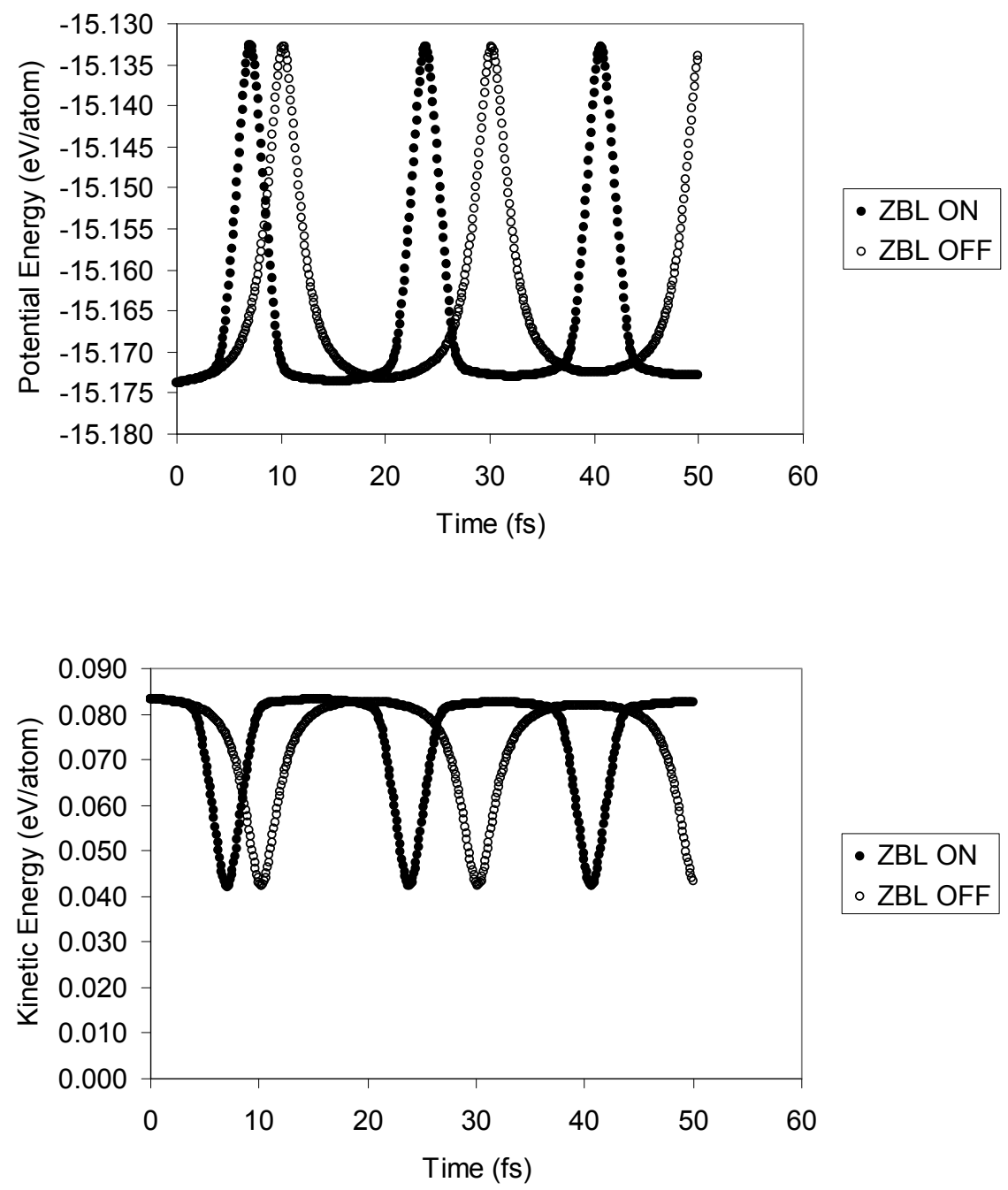

Figure 3-24. Potential and kinetic energies of collision simulations with and without ZBL potential.

\subsubsection{Variable time step}

Even when the interatomic interaction is correct, the interatomic forces can be extremely large. If a normal MD time step of $\sim 1 \mathrm{fs}$ is used, such a large force on an atom can cause the atom to move a very large distance, leading to stability problems in the code. One solution to this to that simulation is performed with extremely short time step size, but it will take unreasonably long time to complete the simulation. A better method to resolve this problem is to allow the time step to change during the course of the simulation. When some atoms 
are energetic, the time step is automatically adjusted to small size, and then once the energy dissipates, the time step size gets increased.

The variable time step algorithm that we have developed is based on the accuracy of the calculation of the trajectory of the energetic atoms. First the most energetic atom in the system is identified by the predictor step, which is nothing but a calculation of the trajectory using the Taylor expansion:

$$
\vec{x}(t+d t)=\vec{x}(t)+\vec{v}(t) d t+\frac{1}{2} \vec{a}(t) d t^{2}+\ldots,
$$

Where $x(t), v(t), a(t)$ are the position, velocity, acceleration of the atom at time $t$. The most energetic atom has the largest predicted displacement. Now the Gear predictor-corrector step is performed to calculate the position of the most energetic atom. It must be noted that the predictor step must be performed on all the atoms within the neighborhood of the most energetic atom in order to obtain the correct force on the energetic atom. Once the new position of the atom is determined, the code performs the same calculation but with two half steps. These results should agree to the accuracy of the integration scheme. The two small steps are expected to be more accurate than the one large step. Therefore, if the difference in the two trajectories is larger than a user defined value, the code will perform the same calculation with smaller time step size until the tolerance level is reached.

In order to establish the validity of the variable time step scheme, a fixed time step simulation with a small time step must be done as a base case scenario. Figure 3-25 shows the potential, kinetic, and total energies of as functions of time, and each color corresponds to a specific time step size indicated in the legend. This simulation was done in $22 \times 22 \times 22$ unit cells (20x20x20 unit cells active atoms) with $1 \mathrm{keV} U$ PKA, which is a typical radiation damage simulation setup. Surprisingly, the variation in these energies is rather small between all the cases. It means that the time step size does not make significant difference in the energetics of the radiation damage simulation. However, the number of defects produced turns out to be quite different (see Fig. 3-26). Both the numbers of uranium and oxygen vacancies produced in the beginning are almost identical even though the time step size varies from 0.50 to $0.01 \mathrm{fs}$. However, during the defect annihilation process, the results start to vary. In particular, the number of oxygen vacancies produced show almost $30 \%$ difference between the highest and lowest at 3 ps. Unfortunately this time step size range was not wide enough to determine the maximum time step size which our result converges to a unique data. Currently the simulation with smaller time step size is in progress.

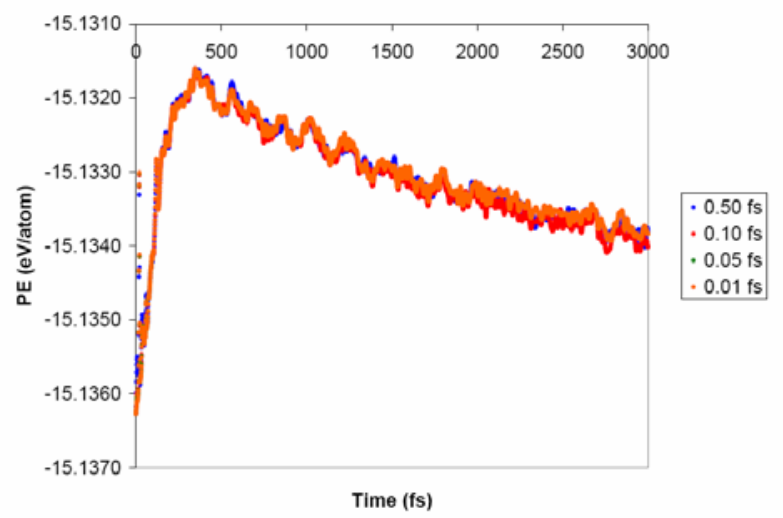



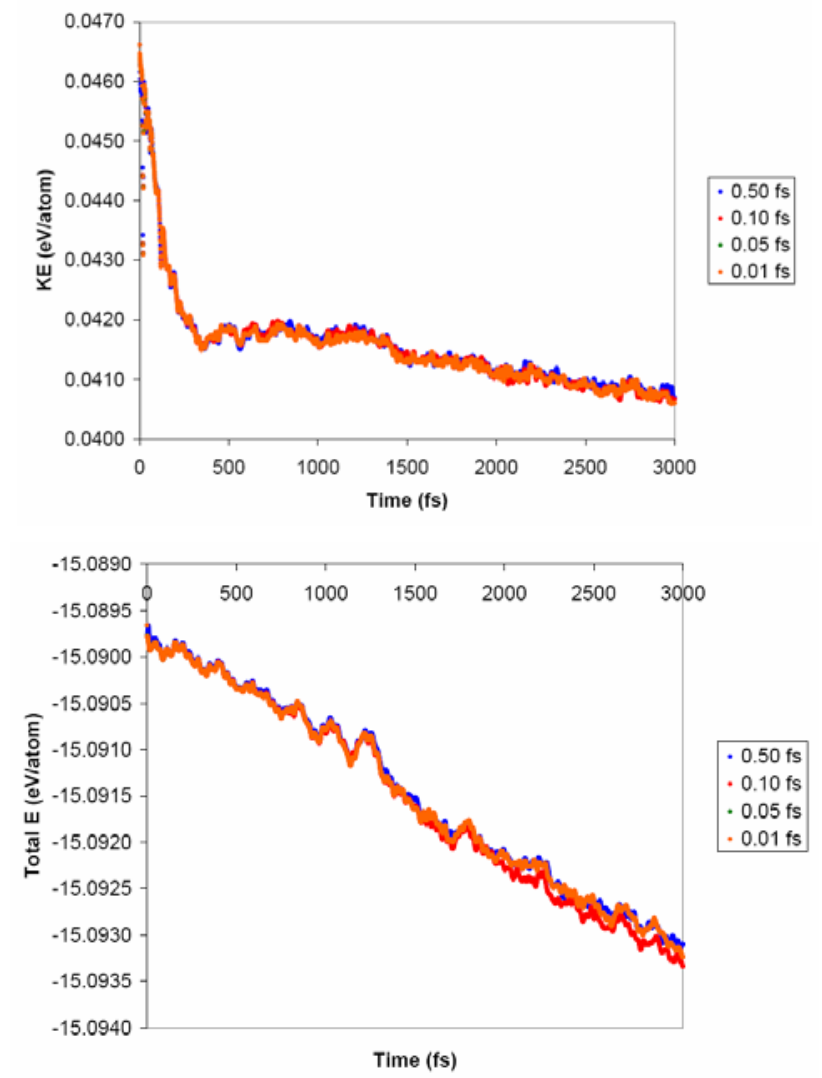

Figure 3-25. Potential (top), kinetic (middle), and total (bottom) energies per atom from a radiation damage simulation with $1 \mathrm{keV} U \mathrm{PKA}$ in [100]. 

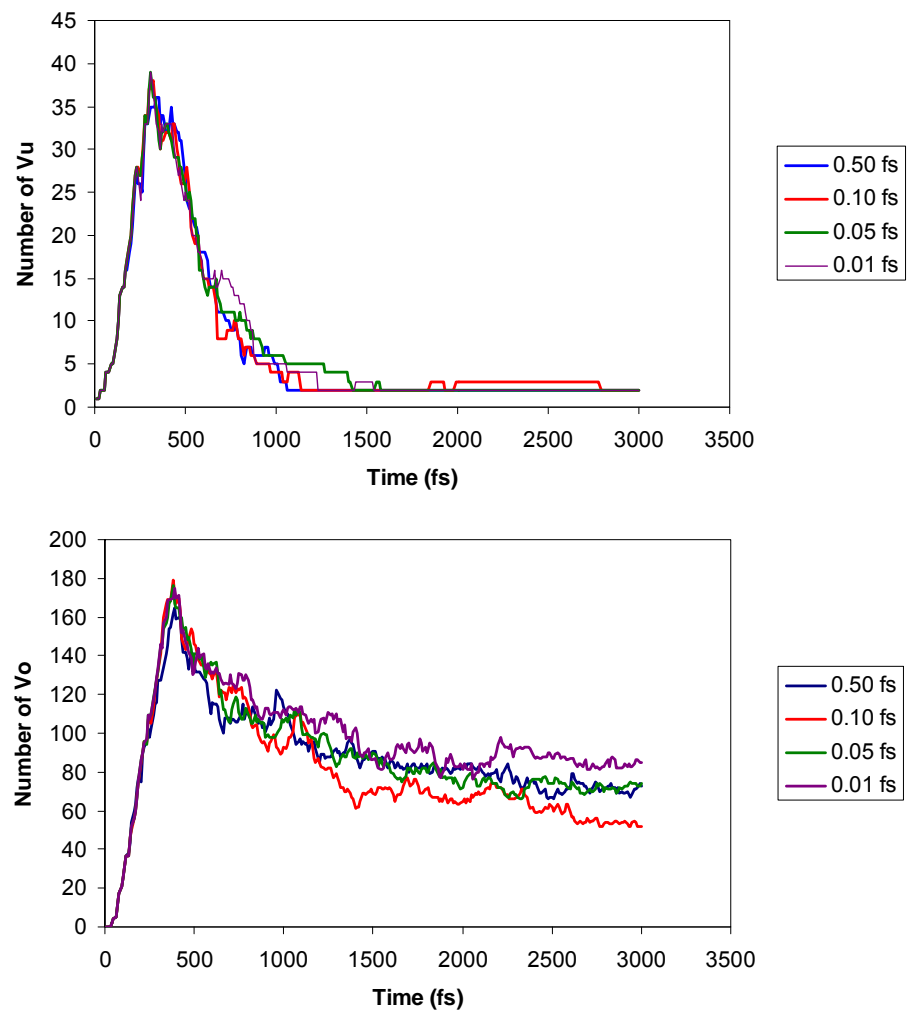

Figure 3-26. Number of uranium (top) and oxygen (bottom) vacancies produced during the radiation damage simulation.

A comparison of the fixed and variable time step runs are shown in Figure. 3-27. The fixed time step simulation data is the same data as the one for $\mathrm{dt}=0.01 \mathrm{fs}$ shown in Figure. 3-25. The variable time step run was performed with the exactly the same configuration except the time step was controlled with the user defined accuracy of $5 \times 10^{-5}$. This accuracy was determined simply by trial and error on the same system for short simulations. The variable time step run actually terminated before 3 ps simply because the ending simulation time cannot be know a priori.

All the energies show good consistency between the fixed and variable time step simulations. The inset of the top panel of Figure. 3-27 shows the potential energy change over the first $200 \mathrm{fs}$. This is the most violent period of the entire simulation and requires very small time step size. The agreement between the fixed and variable time step runs is excellent. Once the base case scenario with the fixed time step simulation is established, the run will be used to compare with the variable time step data. The number of simulation steps needed, and thus the actual computer time required, was considerably smaller for the variable time-step simulation, since during extended periods of the simulation, quite long time steps are acceptable. 

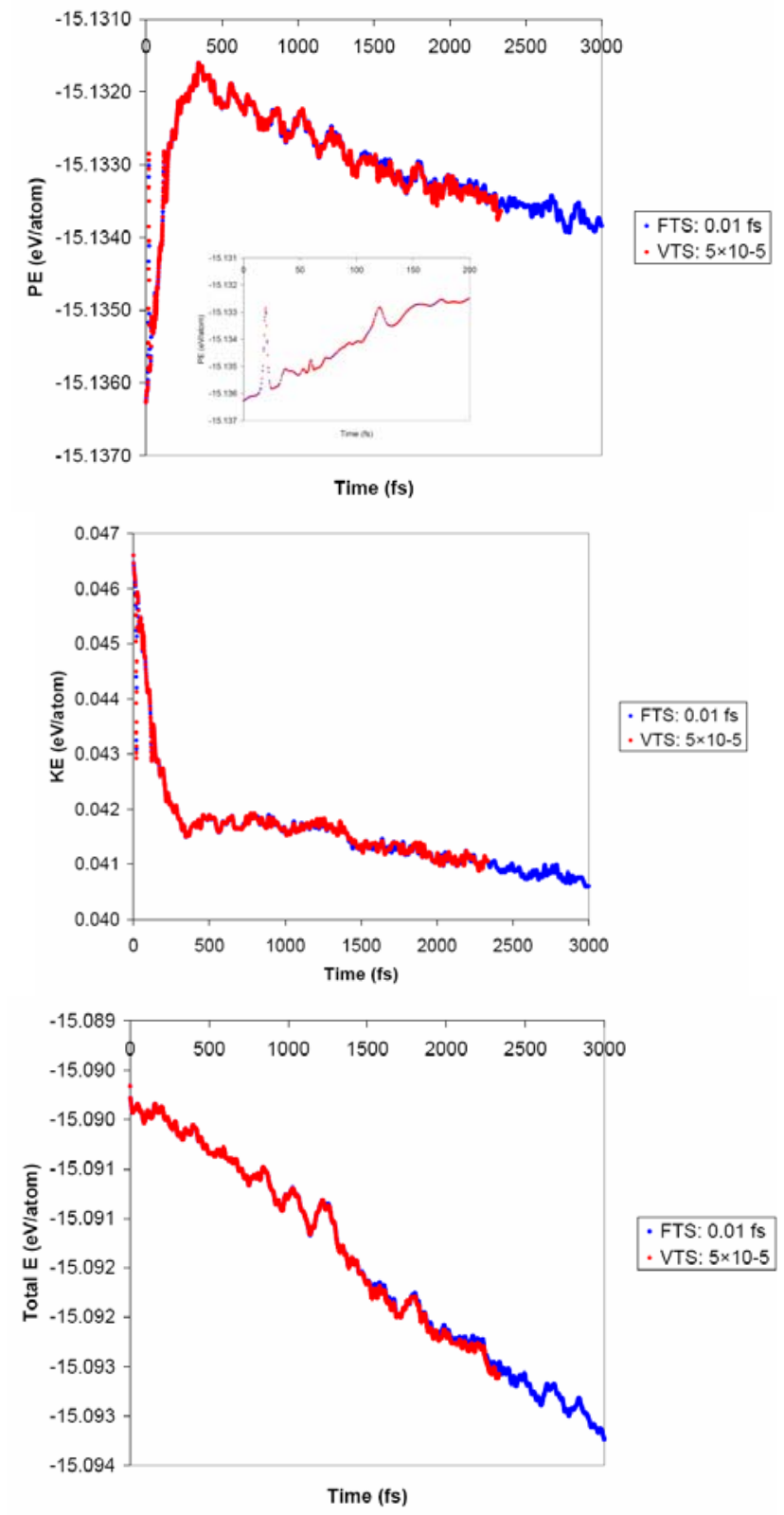

Figure 3-27. Potential (top), kinetic (middle), and total (bottom) energy comparison between the fixed time step with $\mathrm{dt}=0.01 \mathrm{fs}$ and variable time step with error $=5 \times 10^{-5}$. In-set in the top figure shows the potential energies over the first $200 \mathrm{fs}$. 


\subsubsection{Thermostat}

During the radiation damage, large amount energy is converted from the ballistic energy of the PKA to the thermal energy of the entire system. This thermal energy can be overwhelming in the small simulation cell if the heat generated is not appropriately treated. In our simulation cell, a thermostat is applied around the active region of the $\mathrm{UO}_{2}$. Currently the velocity rescaling thermostat is used to control the temperature of the thermostat region. The minimum volume of active and thermostat region must be determined to achieve the maximum efficiency without disturbing the evolution of the cascade. The Langevin thermostat is a stochastic approach to control the system temperature by the use of random forces and damping $^{46,47}$ :

$$
m \vec{a}(t)=\vec{F}(t)-\beta \vec{v}(t)+\vec{R}(t)
$$

This is called the Langevin equation of motion. $a(t)$ is the acceleration, $F(t)$ is the force, $\beta$ is the frictional coefficient, $v(t)$ is the velocity, $R(t)$ is the Gaussian random force of zero mean. The parameters are given by

$$
\begin{aligned}
\beta & =\frac{1}{6} \pi \omega_{D} \\
\omega_{D} & =\frac{k_{B} T_{D}}{\hbar} \\
\sigma^{2} & =\frac{2 m \beta k_{B} T}{\Delta t}
\end{aligned} .
$$

Here $T_{D}$ is the Debye temperature, $k_{B}$ is the Boltzmann constant, $\hbar$ is the Planck constant, $\sigma^{2}$ is the variance of the random force $R(t), m$ is the mass, $\Delta t$ is the time step size. Langevin thermostat is known to correctly sample the canonical ensemble and works well for the radiation damage simulation ${ }^{48}$.

Simulations to equilibrate a single crystal have been performed on $10 \times 10 \times 10$ unit cells single crystal $\mathrm{UO}_{2}$ by choosing all the atoms as thermostat. The simulation was run for $500 \mathrm{fsec}$ with $0.1 \mathrm{fs}$ time step size. Figure 3-28 presents the effect of the target temperature. The Debye temperature was set to $300 \mathrm{~K}$. Starting from $0 \mathrm{~K}$, the system successfully converges to the target temperature. At the highest temperature, the fluctuation is rather large and the temperature slightly overshoots. Figure 3-29 shows the effect of the Debye temperature on the equilibrating the structure. As the Debye temperature increases it takes longer to reach the target temperature because the frictional term contribution becomes stronger. Further test are needed to use this thermostat in the radiation damage simulation, once the appropriate time step size is determined from the base case study. 


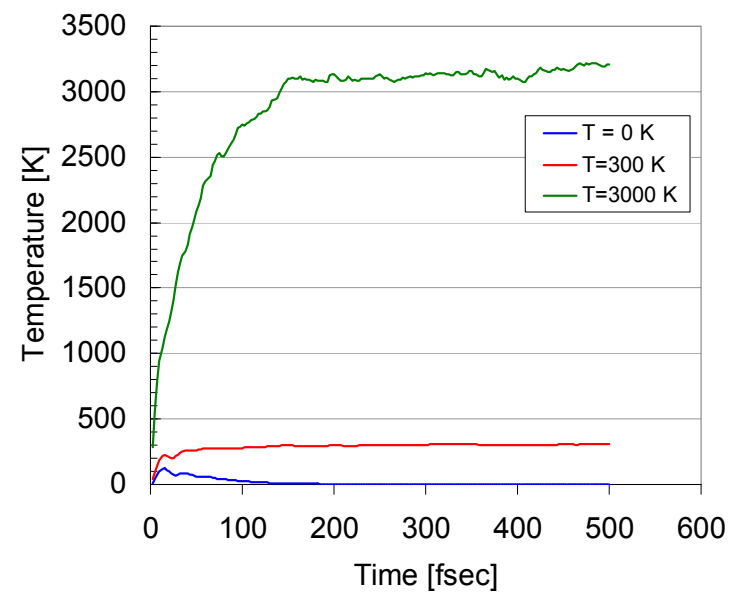

Figure 3-28. Effect of the target temperature in the Langevin thermostat.

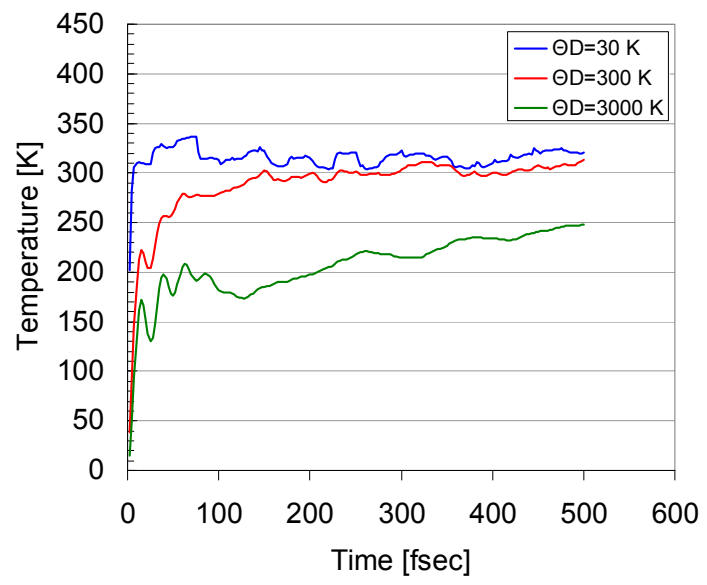

Figure 3-29. Effect of the Debye temperature on the Langevin thermostat.

\section{Phase Behavior}

\subsection{Introduction}

The work on phase diagrams and thermodynamic optimization of systems relevant for nuclear materials included the research on the system Ce-O. A thermodynamic computer dataset was developed which can be used for calculating thermodynamic functions and heterogeneous equilibria. The results were published in the International Journal of Materials Research. Additionally, the experimental and theoretical data for the ternary systems O-Pu$\mathrm{U}, \mathrm{Fe}-\mathrm{O}-\mathrm{U}, \mathrm{O}-\mathrm{Pu}-\mathrm{Zr}$ and $\mathrm{N}-\mathrm{Pu}-\mathrm{U}$ were critically assessed. Two system reports were published for the phase diagrams and crystal chemistry data in the Landolt-Börnstein handbook series. 


\subsection{Results and Discussion}

\subsubsection{Thermodynamic Optimization of the Ce-O System}

In this project the thermodynamic properties and coupled phase diagram of the Ce-O system were investigated by computational thermodynamics using the CALPHAD (CALculation of PHAse Diagrams) method. ${ }^{49}$ Cerium oxide is frequently used as a model material (surrogate) for plutonium oxide. In reducing atmospheres at high temperatures both compounds lose oxygen in a very similar way. The resulting $\mathrm{Ce}$ - and Pu-compounds show a distinct range of stoichiometry and are structurally and thermodynamically closely related.

In the course of the thermodynamic optimization, the solution phases are thermodynamically modeled according to their crystallography and crystal chemistry. The model parameters are adjusted to experimental data, or results from first principle calculations or estimates. A scheme is shown in Figure. 4-1. ${ }^{50}$ Sublattice models are used taking into account the crystallographic site occupancies of the solution phases. Guidelines for the solution phase modeling, the thermodynamic optimization and thermodynamic applications as developed in a series of international workshops ${ }^{51}$ were applied. As shown in Figure. 4-1 the thermodynamic data are stored in computer databases and then can be used for the calculation of phase equilibria and reactions in the multi-component, multiphase systems. The thermodynamic calculations allow taking into account the specific compositions of nuclear materials and environmental conditions of operation. Predictive calculations ("thermodynamic simulations") for compositions and temperature ranges of a system previously not investigated by experiments can be made. The system $\mathrm{Ce}-\mathrm{O}$ was optimized and calculated using the software packages Thermo-Calc/Parrot ${ }^{5,53}$ and the Lukas Programs (BINGSS, BINFKT, TERGSS, TERFKT). ${ }^{54}$ 


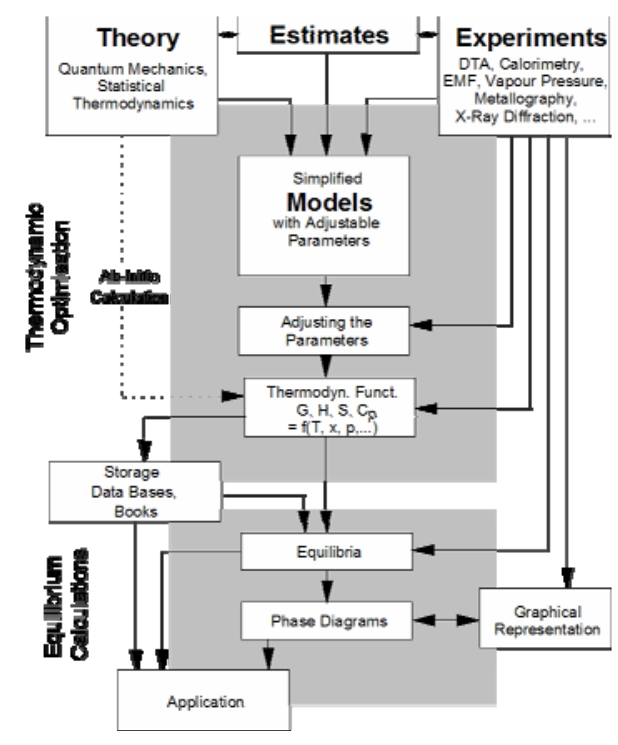

Figure. 4-1. Scheme of the thermodynamic optimization and equilibrium calculations ${ }^{50}$

Numerous experimental data for the $\mathrm{Ce}-\mathrm{O}$ phase diagram and related thermodynamic properties have already been published. Therefore, models and computer datasets with Gibbs free energy descriptions for the Ce-O system phases developed from the CALPHAD approach could be comprehensively tested and compared with available experimental data. Some results of the optimization are compared to experimental values from literature in Figures 4-2 to 4-4. The calculated partial phase diagram of the Ce-O system is shown in Figure. 4-2. This figure refers to the central part of the Ce-O system from 60 to $67 \mathrm{~mol} \% \mathrm{O}$ and up to $2000 \mathrm{~K}$. This kind of calculation required the phase description of all the reported solid phases including the non-stoichiometric $\mathrm{CeO}_{2-x}$ phase and the five intermediate phases. The description was in terms of the temperature dependence of Gibbs free energy for each phase. Figure 4-3 shows the heat capacities of $\mathrm{CeO}_{2}$ and $\mathrm{Ce}_{2} \mathrm{O}_{3}$ between $250 \mathrm{~K}$ and 1250 $\mathrm{K}$. The optimization results reflect the experimental data to an extent. The enthalpy increment of $\mathrm{CeO}_{2}$ is compared to the experimentally measured values] in Figure 4-4. As can be seen, this data is very well reproduced by the optimization 


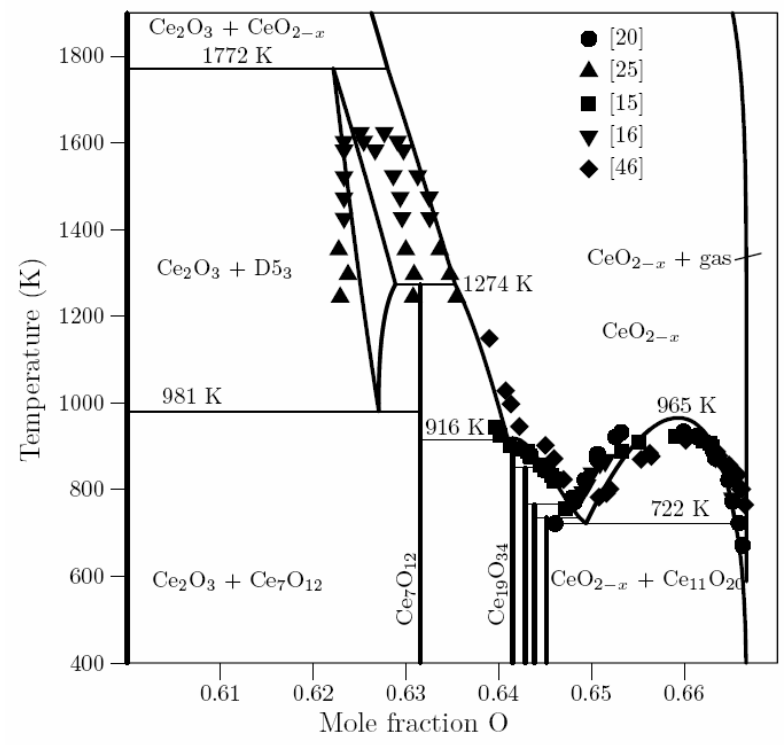

Figure 4-2. Calculated partial phase diagram for the system $\mathrm{Ce}-\mathrm{O}$ in the compositions range $60-67 \mathrm{~mol} \% \mathrm{O}$.

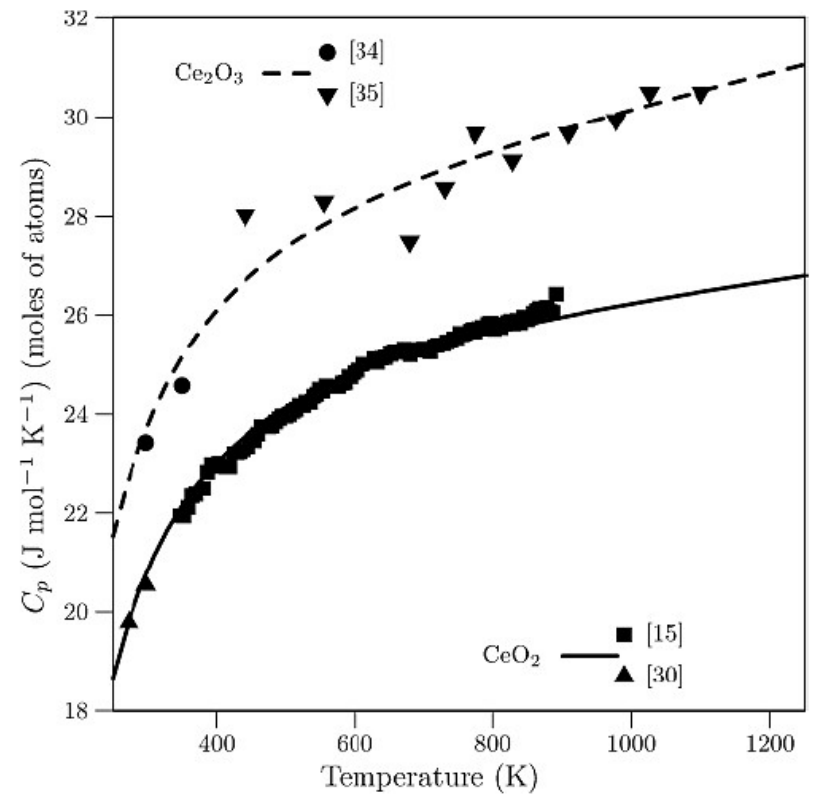

Figure 4-3. Calculated heat capacities of $\mathrm{CeO}_{2}$ and $\mathrm{Ce}_{2} \mathrm{O}_{3}$ 


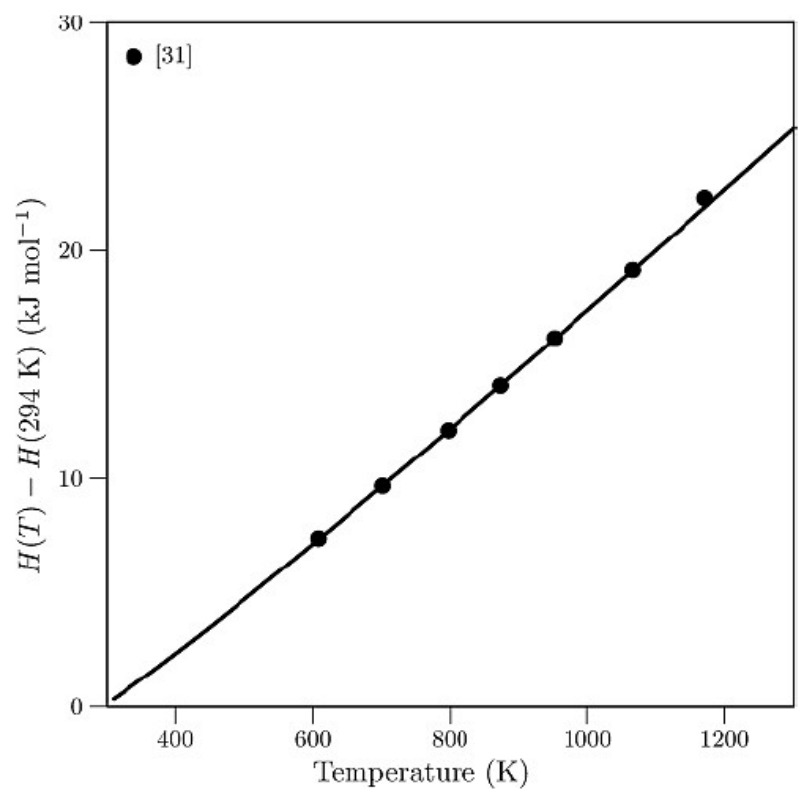

Figure 4-4. Calculated enthalpy increment of $\mathrm{CeO}_{2}$

\subsubsection{Critical assessments (evaluations) of nuclear materials systems}

Critical assessments for three nuclear systems were provided: (1) U-Pu-O, (2) Fe-O-U and (3) O-Pu-Zr. Two of these evaluations (Fe-O-U, O-Pu-Zr) were published in the handbook series Landolt-Bornstein New Series IV/11C4:

The cited volume in the New Series of Landolt-Börnstein provides critically evaluated data on phase diagrams, crystallographic and thermodynamic data of ternary alloy systems. In volume IV/11C, Part 4 selected nuclear materials and engineering systems were considered. The phase diagrams provide materials scientists and engineers with basic information important for fundamental research, development and optimization of materials. The evaluation reports published have undergone a thorough review process in which we had access to all the original publications. The data for each ternary system is provided in a standard format which includes text, tables and diagrams. The topics presented are literature data, binary systems, solid phases, pseudobinary systems, invariant equilibria, liquidus, solidus, and solvus surfaces, isothermal sections, temperature-composition sections, thermodynamics, materials properties and applications, and miscellaneous. Finally, a detailed bibliography of all cited references is provided.

As an example for a result of the evaluation, Figure. 4-5 shows an evaluated $\mathrm{UO}_{\mathrm{x}^{-}}$ $\mathrm{FeO}_{x}$ section in the $\mathrm{Fe}-\mathrm{O}-\mathrm{U}$ system as given in the system report. 


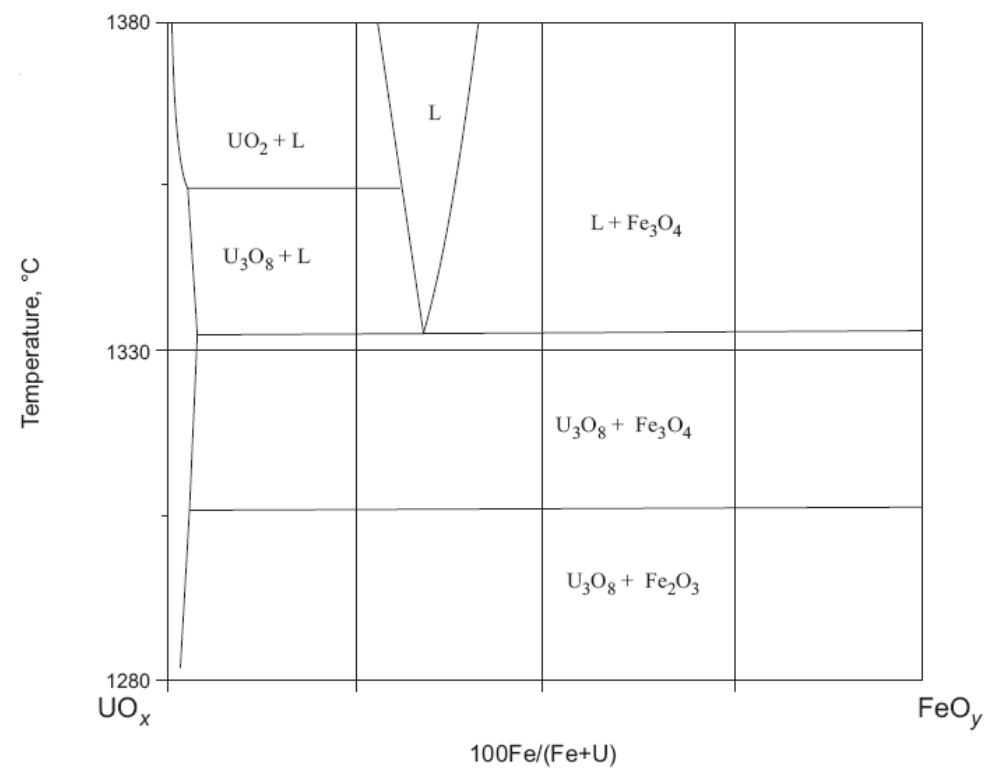

Figure 4-5 Isobaric section under $1173 \mathrm{~Pa}$ of oxygen.

\subsubsection{Experimental literature data for the U-Pu-O system Phase diagram data}

The pseudo-binary $\mathrm{UO}_{2}-\mathrm{PuO}_{2}$ system was investigated by Lyon and Bailey (1967). ${ }^{55}$ Only limited information is available on the phase diagram data for the ternary U-Pu-O system. Just one partial ternary phase diagram indicating equilibria for temperatures of 400,600 and $800^{\circ} \mathrm{C}$ was published ${ }^{56}$ and is shown in Figure. 4-6.

\section{Enthalpy data and heat capacities}

Measurements of enthalpy increments $\left(H_{r}^{0}-H_{2 \% 8}^{0}\right)$ and heat capacities $\left(C_{p}\right)$ are documented in the literature for uranium-plutonium mixed oxides containing 20at.\% and 25at.\% $\mathrm{PuO}_{2}{ }^{57,58}, 59,60,61,62,63,64,65$. There is agreement among the different sets of data for the enthalpy, just the datasets of Leibowitz et al. ${ }^{63,64}$ deviate significantly. Fink ${ }^{13}$, Cordfunke and Konings $^{66}$, "MATPRO" ${ }^{67}$ and Carbajo et al. ${ }^{68}$ have correlated enthalpies and heat capacities for uranium-plutonium mixed oxides. Carbajo et al. ${ }^{68}$ recommended to use the NeumannKopp molar-additivity rule to calculate the enthalpy and heat capacity because of the ideal behavior of the solid solutions. To prove this assumption for solid solutions with plutonium oxide content higher than 25at. \%, Kandan et al. ${ }^{62}$ recently measured the enthalpy increments of $\mathrm{U}_{1-\mathrm{y}} \mathrm{Pu}_{\mathrm{y}} \mathrm{O}_{2}$ solid solutions with $\mathrm{y}=0.21,0.28$ and 0.4 using a high-temperature differential calorimeter in the temperature range 1000-1780 K. From these data, the heat capacity, entropy and the Gibbs energy functions were derived. It was concluded that the enthalpies of $(\mathrm{U}, \mathrm{Pu}) \mathrm{O}_{2}$ solid solutions in fact obey the Neumann-Kopp molar-additivity rule. New relations of density / compressibility, density / temperature, thermal expansion / 
temperature and isothermal compressibility / temperature were proposed for $(\mathrm{U}, \mathrm{Pu}) \mathrm{O}_{2}$ with $\mathrm{y}_{\mathrm{Pu}} \leq 0.25$ by Breitung and Reil. ${ }^{69}$ The ranges of validity are 1400 to $3600 \mathrm{~kJ} / \mathrm{kg}$ and 3120 to $7600 \mathrm{~K}$, respectively. From these results it was concluded that the critical temperature of $(\mathrm{U}, \mathrm{Pu}) \mathrm{O}_{2}$ is well above $8000 \mathrm{~K}$.

\section{Vaporization studies}

Mass spectrometric studies of the vaporization of the $U_{1-\mathrm{y}} \mathrm{Pu}_{\mathrm{y}} \mathrm{O}_{\mathrm{z}}$ system were conducted by Ohse and $\operatorname{Olson}^{70}(y=0.15, z=1.94$ to $2.0 ; T=1800-2350 \mathrm{~K})$ and by Battles et al. ${ }^{71}(\mathrm{y}=0.2$ and $z=1.92$ to $2.0 ; T=1905$ to $2411 \mathrm{~K}$ ). Vapor pressures with a Knudsen effusion cell were measured by Dean et al. ${ }^{72}$ using ${ }^{239} \mathrm{Pu}$ and ${ }^{233} \mathrm{U}(\mathrm{y}=0.5, \mathrm{z}=1.90$ to $2.10 ; \mathrm{T}=1814 \mathrm{~K})$. From these data oxygen potentials can be derived. Investigations of the oxygen potentials were also provided by Chilton and Kirkham, ${ }^{73}$ Chilton and Edwards, ${ }^{74}$ Woodley ${ }^{75,76,77}$ and Woodley and Adamson. ${ }^{78}$

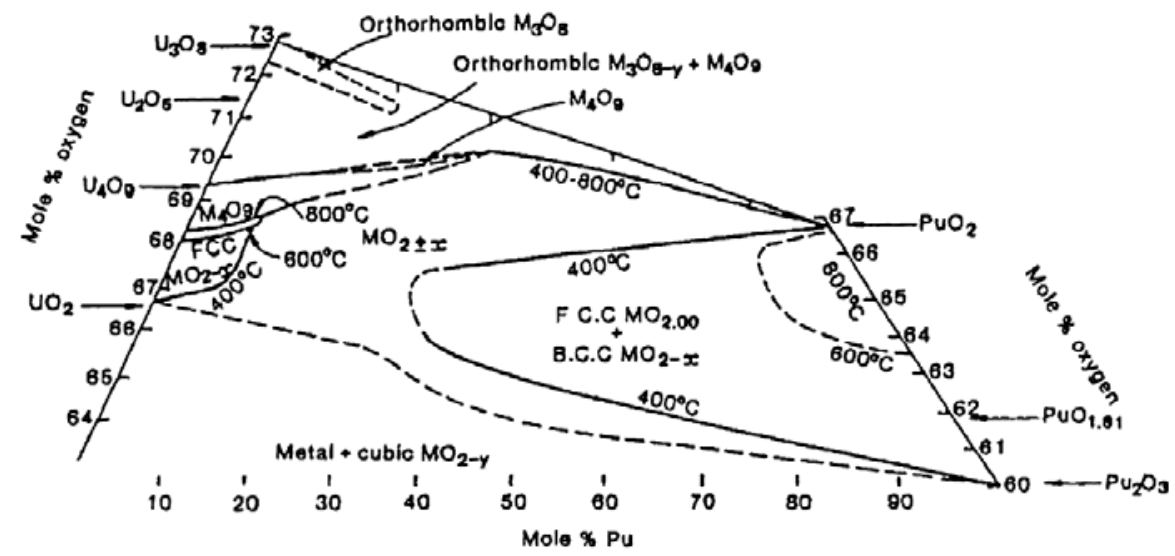

Fig. 4-6. Partial ternary phase equilibria in the U-Pu-O system. ${ }^{56}$

Calculations of the oxygen potentials were performed by Rand and $\operatorname{Markin}^{79}(y=0.15, z=$ 1.95 to $2.10 ; \mathrm{T}=2100 \mathrm{~K})$ by Tetenbaum ${ }^{80}(\mathrm{y}=0.20, \mathrm{z}=1.92$ to $1.96 ; \mathrm{T}=2150-2550 \mathrm{~K})$ and Green et al. ${ }^{81}(y=0-0.3 ; z=1.9-2.00 ; T=2500-6000 \mathrm{~K})$. Recently, also Viswanathan and Krishnaiah ${ }^{82}$ calculated the vaporization of hypo-stoichiometric uranium plutonium dioxide as a function of the $\mathrm{O} / \mathrm{M}(\mathrm{M}=\mathrm{U}+\mathrm{Pu})$ ratio and plutonium content $(\mathrm{y}=0.10,0.15,0.20$, $0.25,0.30,0.35,0.40$ and $0.50, z=1.88$ to $2.00 ; \mathrm{T}=2000 \mathrm{~K}$ ). It was concluded that the deviation from ideality in the $\mathrm{UO}_{2}-\mathrm{PuO}_{2}$ pseudo-binary system is negligible.

With respect to the thermodynamic calculations of the U-Pu-O system, we have focused on performing a detailed assessment of existing thermodynamic data in the U-Pu-O.

Thermodynamic datasets for the binary oxide systems $\mathrm{U}-\mathrm{O}^{83}, \mathrm{Pu}-\mathrm{O}^{84}$ and $\mathrm{Pu}-\mathrm{U}^{85}$ are available from literature. Based on these binary systems a dataset for the ternary U-Pu-O system was developed by Yamanaka et al. ${ }^{86}$ This dataset can be used to calculate 
thermodynamic functions (heat capacities, enthalpies etc) and phase equilibria for nuclear fuel candidate materials in the ternary U-Pu-O system. Besides the fluorite-type solid solution $\mathrm{PuO}_{2-\mathrm{x}}-\mathrm{UO}_{2 \pm x}(\mathrm{MOX}$, Mixed Oxide Fuel) the following phases were taken into account: $\alpha-\mathrm{Pu}$, $\beta-P u, y-P u, \delta-P u, \delta '-P u, \varepsilon-P u, \gamma-U, \alpha-U, \beta-U, \zeta, \eta, P_{2} O_{3}, P_{u} O_{1.52}, \alpha-P_{u} O_{1.61}, U_{4} O_{9}, U_{3} O_{8}$, $\mathrm{UO}_{3}$, liquid phase and gas phase. Based on the available Gibbs free energy data for all these phases, the reactions of nuclear materials in the U-Pu-O system under various physicochemical conditions can be calculated.

However, recently, Labroche et al. ${ }^{87,88}$ published new critical assessments of experimental data for the U-O system and three research groups, Chevalier et al. ${ }^{89}$ and Guéneau et al., ${ }^{90}$ and Lewis et al., ${ }^{91}$ independently published new optimized thermodynamic descriptions for the U-O system. None of these three binary $\mathrm{U}-\mathrm{O}$ datasets was used to update the thermodynamic description for the U-Pu-O system. Yamanaka et al. ${ }^{86}$ also did not take into account all of the published experimental data for the optimization of the thermodynamic functions and the coupled phase diagram.

We have prepared a report on the U-Pu-O ternary system. This report requires the collection of all the available literature for this system regarding thermodynamics, structure and other studies carried out (Table 4-1). Our critical assessment allowed us to present isothermal sections in the ternary phase diagram as presented in Fig. 4-7. The report includes data about the isothermal sections, quasi binary systems, invariant equilibria, and solidus surfaces. The final part is the assessment of thermodynamic data to develop a computer dataset for calculations. The assessment has been done under the international guidelines set by MSIT (Materials Science International Team and Landolt-Bornstein Handbook series) Ternary Evaluation Program. 


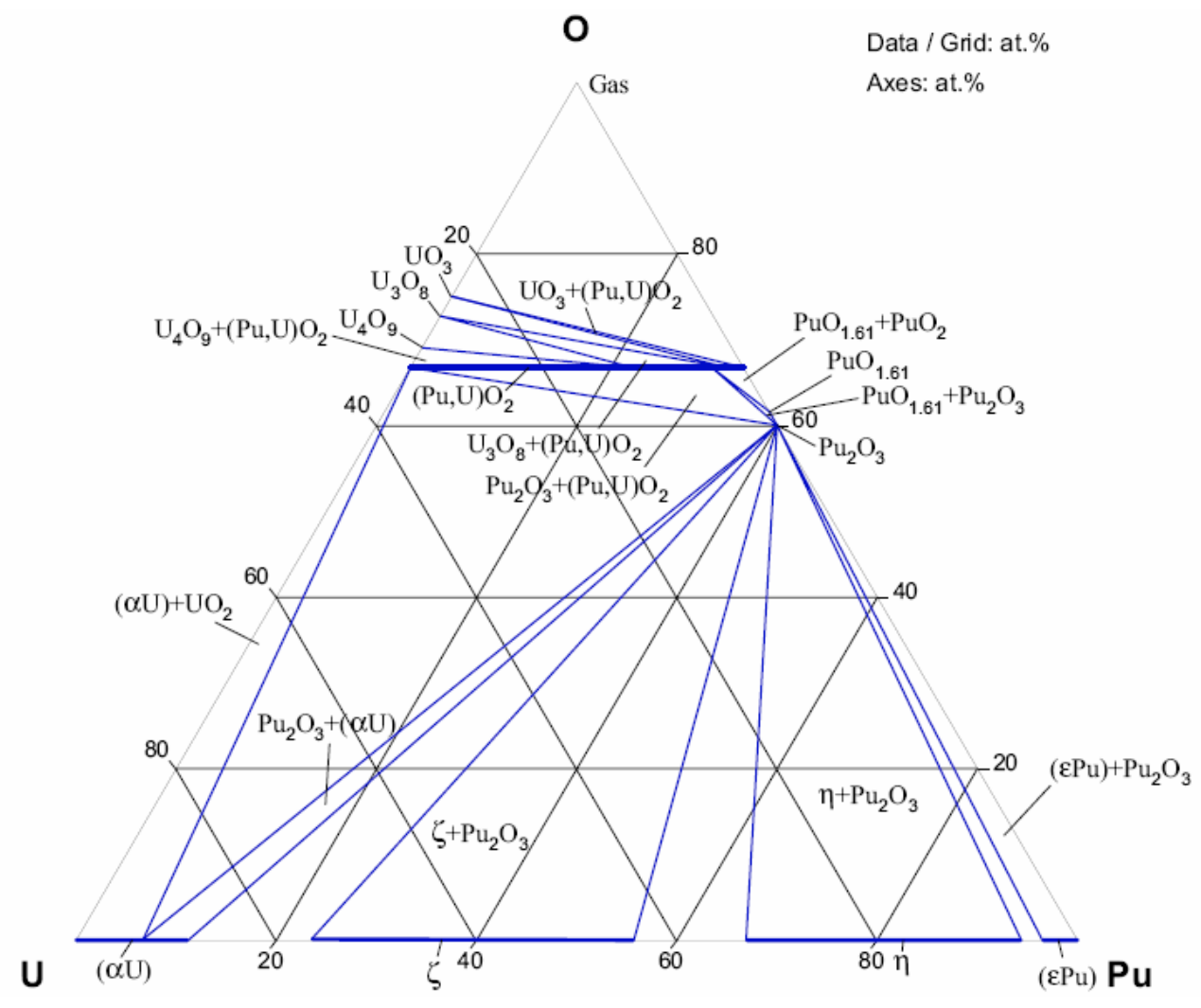

Figure 4-7. Optimized O-Pu-U Isothermal Section at $1000^{\circ} \mathrm{C}$, determined from our critical assessment of the literature. 
Table 4-1: Investigations of the O-Pu-U Phase Relations, Structures and Thermodynamics

\begin{tabular}{|c|c|c|}
\hline Reference & Method/Experimental Technique & $\begin{array}{l}\text { Temperature/Composition/Phase Range } \\
\text { Studied }\end{array}$ \\
\hline [1958Eli] & $\begin{array}{l}\text { Chemical analysis, heat treatment, thermal } \\
\text { analysis, dilatometry, } \\
\mathrm{X} \text { ray diffraction, metallography }\end{array}$ & $600-700^{\circ} \mathrm{C}$ for $300 \mathrm{~h} / \mathrm{Pu}-\mathrm{U}$ \\
\hline [1967Ack] & $\begin{array}{l}\text { X-ray diffraction, metallography } \\
\text { examination, emf }\end{array}$ & $\begin{array}{l}\text { up to } 1000^{\circ} \mathrm{C} / 64 \text { to } 73 \text { at. } \% \mathrm{O} \text { and } 40 \text { to } 33 \\
\text { at. } \% \mathrm{Pu}\end{array}$ \\
\hline [1967Lyo] & $\begin{array}{l}\text { Standard gravimetric analyses, } \mathrm{X} \text { ray } \\
\text { fluorescence and } \mathrm{X} \text { ray diffraction }\end{array}$ & $2300-2900^{\circ} \mathrm{C} / 5$ to $85 \mathrm{~mol}^{2} \mathrm{PuO}_{2}$ \\
\hline [1968Sar] & Metallography, X-ray diffraction & $\begin{array}{l}\text { up to } 1600^{\circ} \mathrm{C} / \mathrm{PuO}_{2-x}, 1.62 \leq x \leq 2.00 \\
(\mathrm{Pu}, \mathrm{U}) \mathrm{O}_{2}\end{array}$ \\
\hline [1969Koil] & $\begin{array}{l}\text { Differential thermal analysis, } \\
\text { X-ray diffraction }\end{array}$ & $\begin{array}{l}25-800^{\circ} \mathrm{C} /\left(\mathrm{Pu}_{y} \mathrm{U}_{1-y}\right) \mathrm{O}_{2-x}, \\
0.17 \leq y \leq 0.40 \\
1.92 \leq x \leq 2.00\end{array}$ \\
\hline [1970Bat] & $\begin{array}{l}\text { Mass-loss and mass-spectrometric Knudsen } \\
\text { effusion }\end{array}$ & $1632-2138^{\circ} \mathrm{C} / \mathrm{U}_{0.8} \mathrm{Pu}_{0.2} \mathrm{O}_{2-\mathrm{x}}$ \\
\hline [1972Lei] & Calorimetry & $2075-2732^{\circ} \mathrm{C} /\left(\mathrm{Pu}_{0.2} \mathrm{U}_{0.8}\right) \mathrm{O}_{1.97}$ \\
\hline [1973Aff] & Pulse heating method & up to $2727^{\circ} \mathrm{C} /\left(\mathrm{Pu}_{0.2} \mathrm{U}_{0.8}\right) \mathrm{O}_{2 \pm x}$ \\
\hline$[19760 \operatorname{sh} 1]$ & Laser pulse heating & up to $4727^{\circ} \mathrm{C} / 20 \mathrm{~mol}^{2} \mathrm{PuO}_{2}$ \\
\hline [19760sh2] & Laser pulse heating & up to $6727^{\circ} \mathrm{C} / 20 \mathrm{~mol} \% \mathrm{PuO}_{2}$ \\
\hline [1976Tet] & Transpiration method & $1877-2177^{\circ} \mathrm{C} /\left(\mathrm{Pu}_{0.2} \mathrm{U}_{0.8}\right) \mathrm{O}_{2-x}$ \\
\hline [1977Bab] & Laser surface heating method & $\begin{array}{l}\text { up to } 4727^{\circ} \mathrm{C} / \text { liquid } \mathrm{UO}_{2} \text { and } \\
\left(\mathrm{Pu}_{0.2} \mathrm{U}_{0.8}\right) \mathrm{O}_{2}\end{array}$ \\
\hline [1977Tet] & Equilibration technique & $\begin{array}{l}1777^{\circ} \mathrm{C} /\left(\mathrm{Pu}_{y} \mathrm{U}_{1-y}\right) \mathrm{O}_{2-x}, 0.15 \leq y \leq 0.30 \\
1.92 \leq x \leq 2.00\end{array}$ \\
\hline [19780hs] & Laser surface heating method & $\begin{array}{l}\text { up to } 4727^{\circ} \mathrm{C} / \text { liquid } \mathrm{UO}_{2} \text { and } \\
\left(\mathrm{Pu}_{0.2} \mathrm{U}_{0.8}\right) \mathrm{O}_{2}\end{array}$ \\
\hline [1979Woo] & $\begin{array}{l}\text { Thermogravimetric analysis, } \\
\text { solid-electrolyte galvanic cell technique }\end{array}$ & $800-1000^{\circ} \mathrm{C} / \mathrm{Pu}_{0.25} \mathrm{U}_{0.75} \mathrm{O}_{2 \pm x}$ \\
\hline [1981Woo] & $\begin{array}{l}\text { Mass spectrometry analyses, oxygen } \\
\text { potential-composition measurements }\end{array}$ & $\begin{array}{l}1000-1200^{\circ} \mathrm{C} / \mathrm{Pu}_{0.1} \mathrm{U}_{0.9} \mathrm{O}_{2-x}, \\
\mathrm{Pu}_{0.4} \mathrm{U}_{0.6} \mathrm{O}_{2-x}, \mathrm{PuO}_{2-x}\end{array}$ \\
\hline [2004Kan] & Inverse drop calorimetry technique & $\begin{array}{l}727-1507^{\circ} \mathrm{C} /\left(\mathrm{Pu}_{y} \mathrm{U}_{1-y}\right) \mathrm{O}_{2}(y=0.21,028 \\
\text { and } 0.4)\end{array}$ \\
\hline
\end{tabular}




\subsubsection{Assessment of the U-Pu-N System}

In relation to the assessment of the U-Pu-N system, progress has been made on the binary $\mathrm{U}-\mathrm{Pu}$ sub system. Experimental data for this system is in the form of thermodynamic properties for individual phases. This data is used to model the system using CALPHAD taking into account the interaction between phases and the calculated properties can be compared to the experimentally available ones. The first step was to prepare a system report describing the important phase relations and existing thermodynamic data similar to the $\mathrm{N}$ $\mathrm{Pu}-\mathrm{U}$ system (Table 4-2). This work is continued.

Table 4-2: Investigations of the N-Pu-U Phase Relations, Structures and Thermodynamics

\begin{tabular}{lll}
\hline Reference & Method/Experimental Technique & $\begin{array}{l}\text { Temperature/Composition/Phase Range } \\
\text { Studied }\end{array}$ \\
\hline [1963Ans] & $\begin{array}{l}\text { Metallography } \\
\text { X-ray powder diffraction, chemical } \\
\text { analysis }\end{array}$ & $900^{\circ} \mathrm{C} /\left(\mathrm{Pu}_{x} \mathrm{U}_{1-x}\right) \mathrm{N}$ \\
\hline [1971Ale] & Oelsen calorimeter, masspectrometry & $27-2127^{\circ} \mathrm{C} /(\mathrm{Pu}, \mathrm{U}) \mathrm{N}$ \\
\hline [1971Ten] & X-ray measurements, chemical analysis & $\begin{array}{l}1400-1800^{\circ} \mathrm{C} \text { for } 325 \text { minutes } / \\
\left(\mathrm{Pu}_{x} \mathrm{U}_{1-x}\right) \mathrm{N}\end{array}$ \\
\hline [1991Suz] & X-ray diffraction & $1752^{\circ} \mathrm{C} /\left(\mathrm{Pu}_{x} \mathrm{U}_{1-x}\right) \mathrm{N}$ \\
\hline [1992Suz] & $\begin{array}{l}\text { Knudsen effusion mass spectrometry, } \\
\text { X-ray diffraction }\end{array}$ & $1477-1727^{\circ} \mathrm{C} /\left(\mathrm{Pu}_{x} \mathrm{U}_{1-x}\right) \mathrm{N}$ \\
\hline
\end{tabular}

Our critical assessment determined that the calculated phase diagram of U-Pu (Figure. 4-8) is the best fit to the reliable experimental data.

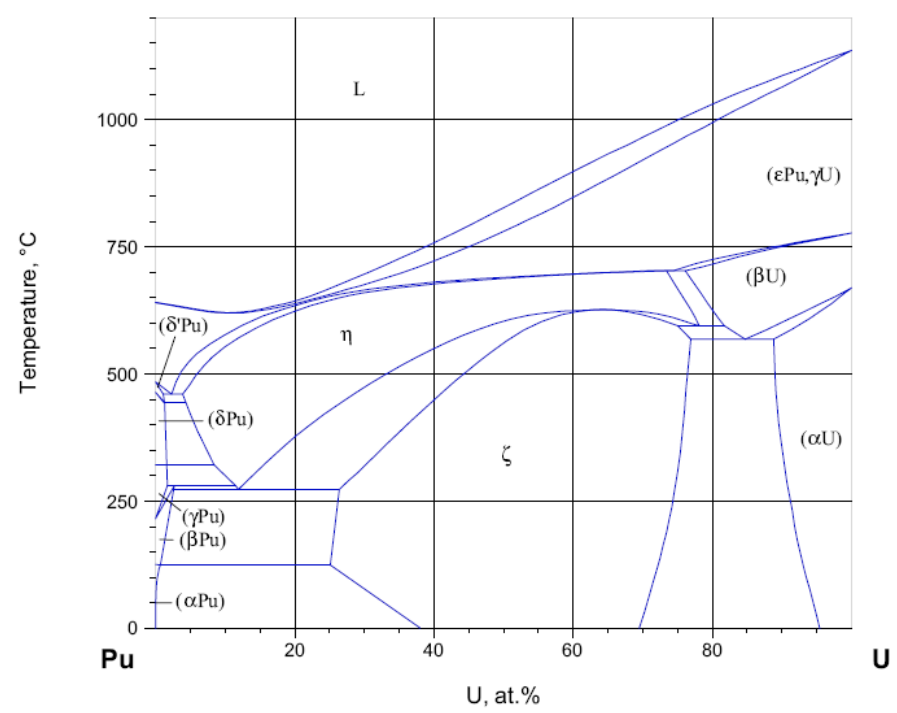

Figure 4-8: The optimal U-Pu binary phase diagram [Kurata, M., "Thermodynamic 


\section{Point Defect Behavior}

\subsection{Methodology Overview}

In this area, we have explored the effect of charge on the point defect formation energies in $\mathrm{UO}_{2}$. We have used the SP-GGA+U $\mathrm{U}^{92}$ method as implemented in Vienna Ab-Initio Simulation Package (VASP) as it predicts the correct insulating ground state of $\mathrm{UO}_{2}$ and allows us to consider charged defects. It is usual to think of point defects in metal oxides as being charged rather than neutral. However, most calculations of the formation energies of point defects treat them as neutral. For example, for an n-type defect the Fermi level will be near the conduction band, while for a p-type defect it will be near the valence band. The most stable defect is that for which the defect energy is lowest for a specific Fermi level. The formation energy of a defect as a function of temperature, partial pressure, defect species $\alpha$, and charge state $\mathrm{q}$ is given as ${ }^{93}$ :

$$
\Delta G_{f}(\alpha, q, T, P)=E^{\text {total }}(\alpha, q)-E^{\text {total }}(\text { perfect })-\Sigma n_{i} \mu_{i}(T, P)+q\left[E_{F}+E_{V}+\Delta V\right]
$$

Here $E^{\text {total }}(\alpha, q)$ is the optimized energy of the supercell containing the defect $\alpha$ of charged state $q$; $E^{\text {total }}$ (perfect) is the optimized energy of the perfect supercell. Both these values are obtained directly from the DFT calculations. In Eq. (5-1), $n_{i}$ represents the number of atoms of species $i$ added to $\left(n_{i}>0\right)$ or subtracted from $\left(n_{i}<0\right)$ the system; $\mu_{i}$ is the chemical potential of species $\mathrm{i}$. The last term is as an electronic chemical potential that represents the change in energy associated with charged defects. In this term, $E_{F}$ is the Fermi level in the bulk with reference to the valence band maximum and $E_{V}$ is the energy of the bulk valence band maximum. The Fermi energy is treated as a variable in this approach and is dependent upon the charge associated with the cumulative effect of defects and dopants in the system. A physically meaningful range around the Fermi energy of pristine $\mathrm{UO}_{2}$ is considered.

The energies of all the individual point defects considered are shown in Figure. 5-1 as a function of the position of the Fermi energy. When considering only the vacancy defects, the +2 charged oxygen vacancy is predicted to be the most stable defect near the valence band. This, in turn, predicts that even in the presence of a uranium vacancy, which is a $p$ type defect, the system will still donate electrons. However, as the Fermi level approaches the conduction band, the -4 charged uranium vacancy becomes increasingly favorable and remains the dominant defect over the range from 0.3 to $2.0 \mathrm{eV}$. When considering only differently charged uranium vacancies, the -4 charged vacancy is the most favorable. The formation energy of this charged vacancy is significantly lower than that of the neutral vacancy. We also examined the effect of charge on the oxygen interstitial (see Figure. 5-1). We did not consider the effect of charge on uranium interstitials because the formation energy of the Frenkel pair is so high. Finally, we observe that the -2 charged oxygen interstitial $\left(\mathrm{O}_{\mathrm{i}}{ }^{\prime}\right)$ dominates over the entire range of the Fermi energies considered, but increases in stability as the Fermi energy approaches the conduction band. 


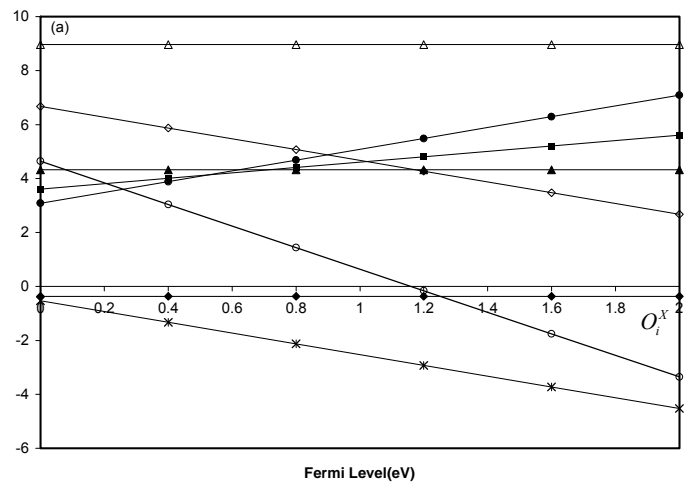

Fig 5-1. Calculated defect formation energies for variously charged oxygen and uranium point defects. The empty symbols denote uranium while the filled symbols are oxygen.

This analysis of the relative stabilities and behavior of individual point defects is informative. However, point defects do not occur in isolation but rather in combinations, such as Frenkel, anti-Frenkel and Schottky complexes. We therefore now compare the energies associated with complexes that consist of either a combination of neutral or charged defects. Because the complexes are charge neutral, the position of the Fermi level does not enter the calculations, even though the energy of the individual charged defects varies with the Fermi level. The formation energy of the anti-Frenkel complex is given in Figure. 5-2. It is clear that the charges on the individual defects that make up the complex influence its overall formation energy. Specifically, the combination of an oxygen anti-Frenkel pair of charged defects $\left(\mathrm{V}_{\ddot{O}}+\mathrm{O}_{i}\right)$ has lower formation energy than the corresponding combination of neutral point defects $\left(V_{O}^{x}+O_{i}^{x}\right)$. The combination of charged components yields a formation energy that is considerably lower than the experimental value.

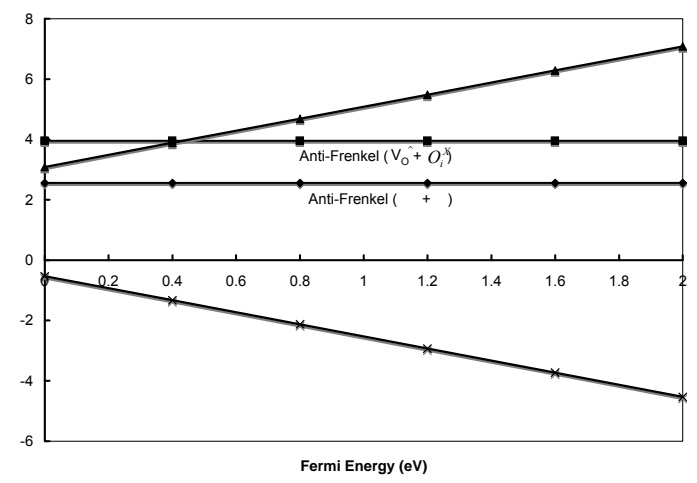

Figure 5-2. Calculated formation energy of the anti-Frenkel defect as a function of the Fermi level; the formation energies of the neutral complexes are compared to charged individual defects.

\subsection{Fission Product Behavior}


In this section, the stabilities in $\mathrm{UO}_{2}$ of selected fission products, $\mathrm{Xe}, \mathrm{Cs}$, and $\mathrm{Sr}$, are investigated as a function of non-stoichiometry. In particular, the incorporation and solution energies of these fission products in $\mathrm{UO}_{2 \pm x}$ are calculated at the anion and cation vacancy sites, at the divacancy and at the bound Schottky defect sites. Fission products can form bubbles which lead to considerable swelling of the fuel and severely degrades mechanical properties, cause corrosion of cladding materials and lead to formation of secondary phases within the fuel. In these calculations, we have used the SP-GGA+U and the non-spin polarized GGA approach as implemented in VASP.

In order to investigate the stabilities of fission products in $\mathrm{UO}_{2}$, it is important to understand some definitions. The first is the incorporation energy. It is the energy required to take a fission product from infinity and place it at an existing trap site. The incorporation energy of a fission product at defect species $\alpha$ is defined $a^{94}$ :

$$
E_{\text {inc. }}=E^{\text {total }}(\alpha)-E^{\text {total }}(\text { defect })-E_{i}
$$

Here $E^{\text {total }}(\alpha)$ is the total energy of the cell with the fission product at a particular defect site, $E^{\text {total }}$ (defect) is the total energy of the cell with a particular defect, $E_{i}$ is the energy of a single isolated fission product. This energy does not account for the formation of the trap site and assumes that there is always an excess of available sites.

The incorporation energy of $\mathrm{Xe}, \mathrm{Cs}$ and $\mathrm{Sr}$ is reported in Table 1 using SP-GGA+U and GGA, and is compared to empirical potential data. ${ }^{94}$ There is overall general agreement between all three methods. First considering the inert fission gas (Xe), all the methods predict the bound Schottky defect to be the most stable incorporation site. The trend can be related to the relative size of the incorporation sites. The bound Schottky defect has the largest size compared to other defects even though it has high formation energy. However, the incorporation energy, by definition, does not account for the formation of a defect.

When considering the incorporation of cesium, there is an apparent discrepancy between SP-GGA+U and GGA. The SP-GGA+U method predicts the uranium vacancy to be the most stable incorporation site while GGA predicts it to be the bound Schottky defect. The $S P-G G A+U$ results are in agreement with empirical potential results. The apparent discrepancy can be understood by taking Coulombic effects into account. The $\mathrm{Cs}^{+}$ion would prefer to stay on a uranium vacancy $\left(\mathrm{U}^{4+}\right)$ since the Coulombic forces are stronger. Since GGA does not predict an ionic or insulating ground state for $\mathrm{UO}_{2}$, it only partially captures these Coulombic effects. These effects are even stronger in the case of a strontium $\left(\mathrm{Sr}^{2+}\right)$ ion and hence all the methods predict the uranium vacancy to be the most stable incorporation site.

Table 5-1. Incorporation energies of xenon, cesium and strontium calculated using SP$\mathrm{GGA}+U$ and GGA and compared to previous empirical potential results. For each of the fission product, the lowest incorporation energy is shown in bold.

\begin{tabular}{|l|r|r|r|}
\hline & SP-GGA+U & GGA & Busker $^{94}$ \\
\hline Xe & & & \\
\hline Interstitial & 11.11 & 12.8 & 17.23 \\
Oxygen vacancy & 9.5 & 9.71 & 13.34 \\
Uranium vacancy & 2.5 & 6.04 & 4.99 \\
Divacancy & 2.45 & 3.29 & 2.84
\end{tabular}




\begin{tabular}{|l|r|r|r|} 
Schottky & $\mathbf{1 . 3 8}$ & $\mathbf{2 . 1 2}$ & $\mathbf{1 . 1 6}$ \\
\hline Cs & & & \\
\hline Interstitial & 10 & 10.1 & 9.93 \\
Oxygen vacancy & 8.4 & 8.1 & 9.1 \\
Uranium vacancy & -3.4 & 0.75 & -6.08 \\
\cline { 4 - 4 } Divacancy & -1.99 & 0.23 & -5.63 \\
\hline Schottky & -0.8 & $-\mathbf{0 . 3 8}$ & -5.47 \\
\hline Sr & & & \\
\hline Interstitial & 4.68 & 4.3 & -11.04 \\
\hline Oxygen vacancy & 7.18 & 5.3 & -8.87 \\
Uranium vacancy & $-\mathbf{9 . 6 6}$ & $-\mathbf{- 5 . 4}$ & $\mathbf{- 2 7 . 0 9}$ \\
Divacancy & -7.53 & -4.97 & -25.31 \\
Schottky & -4.55 & -4.74 & -23.36 \\
\hline
\end{tabular}

Solid fission products such as cesium and strontium can react with oxygen in the fuel and form separate oxides. Any oxide (for example strontium oxide) before solution into the $\mathrm{UO}_{2}$ matrix will decompose into the fission product and oxygen by the following reaction:

$$
\mathrm{SrO}(\mathrm{s}) \longrightarrow \mathrm{Sr}+\mathrm{O}(\mathrm{g})
$$

Therefore, a second definition of energy is required, which is defined as:

$$
E_{S r O}^{\text {solution }}=E_{S r}^{\text {solution }}+E_{O}^{\text {solution }}-E_{S r O}^{\text {formation }}
$$

The first term on the right hand side of the equation is the solution energy of the particular fission product at the most stable trap site based on stoichiometry; the second term is the solution energy of the oxygen from the fission product oxide into any vacant oxygen site in the $\mathrm{UO}_{2}$ matrix. This vacant site also depends on stoichiometry. In $\mathrm{UO}_{2-x}$, the oxygen will be soluble in an oxygen vacancy site, while in $\mathrm{UO}_{2+x}$, it will be present as an oxygen interstitial site, and in $\mathrm{UO}_{2}$, the most stable solution site will be a mixture of vacancy and interstitial sites. The third term is the formation energy of the oxide. If the fission product oxide solution energy is negative, it means that it is energetically favorable for the oxide to be soluble in the fuel. This in turn means that the fission product will not form a stable second phase. Solution energies of oxides of cesium and strontium calculated using SP-GGA+U and GGA and compared to previous empirical potential results. A positive energy implies insolubility in the fuel matrix phase.

Table 5-2 Solution energies of oxides of cesium and strontium calculated using SP-GGA+U and GGA and compared to previous empirical potential results. A positive energy implies insolubility in the fuel matrix phase.

\begin{tabular}{|l|l|l|l|l|l|l|}
\hline & SP-GGA+U & & & Busker $^{94}$ & & \\
\hline
\end{tabular}




\begin{tabular}{|l|r|r|r|r|r|r|} 
Oxide & $\begin{array}{l}\mathrm{UO}_{2-x} \\
\left(\mathrm{UO}_{1.9}\right)\end{array}$ & $\mathrm{UO}_{2}$ & $\begin{array}{l}\mathrm{UO}_{2+x} \\
\left(\mathrm{UO}_{2.1}\right)\end{array}$ & $\mathrm{UO}_{2-x}$ & $\mathrm{UO}_{2}$ & $\mathrm{UO}_{2+\mathrm{x}}$ \\
\hline $\mathrm{SrO}$ & -0.85 & -1.3 & -3.09 & 2.43 & 0.48 & -2.93 \\
\hline $\mathrm{Cs}_{2} \mathrm{O}$ & 2.07 & 2.23 & 0.73 & 10.58 & 8.98 & -1.25 \\
\hline
\end{tabular}

The oxygen solution energies are reported in Table 5-2. A positive value corresponds to the oxide being insoluble in the fuel and forming a second stable phase. The first thing to observe is that oxides with higher oxygen-to-metal ratio are more soluble in the fuel. This can be understood on the basis of their ability to donate oxygen to the lattice. The solubility also increases as the hyperstoichiometry of the $\mathrm{UO}_{2}$ increases. Considering individual oxides, $\mathrm{Cs}_{2} \mathrm{O}$ is predicted to be stable at all stoichiometries, though in $\mathrm{UO}_{2+x}$, it is on the borderline of stability. With regard to $\mathrm{UO}_{2+\mathrm{x}}$, it is also observed experimentally ${ }^{95}$ that the solubility of $\mathrm{Cs}$ increases as we go from hypostoichiometric to hyperstoichiometric fuels. Our calculations support this argument. This can again be related to the argument that the more oxygen an oxide donates to the fuel matrix, the more soluble it is.

The maximum solubility of $\mathrm{SrO}$ in $\mathrm{UO}_{2}$ was experimentally ${ }^{95}$ found to be much higher in $\mathrm{UO}_{2}$ than in $\mathrm{UO}_{2-\mathrm{x}}$, which mirrors our calculated trend. This can be explained on the fact that the solution of strontium becomes increasingly favorable as one approaches hyperstoichiometric conditions owing to the strong Coulombic interactions. The higher charged uranium vacancy $(4+)$ is the most stable solution site in $\mathrm{UO}_{2}$ as compared to the divacancy $(2+)$ site in $\cup_{2-x}$ and hence has a strong preference for charged cations. These studies can be extended to include other fission products and microstructural features such as grain boundaries and can lead to a fundamental understanding of nuclear fuel phenomena.

\section{Papers, Reports, Theses and Presentations}

\section{NERI funded individuals given as bold.}

\section{Refereed Journal Papers:}

- T. Watanabe, S. B. Sinnott, J. S. Tulenko, R. W. Grimes, P. K. Schelling and S. R. Phillpot, "Thermal transport properties of uranium dioxide by molecular dynamics simulation”, J. Nucl. Mater. $\underline{375}$, 388-396 (2008)

- P. Nerikar, T. Watanabe, J. S. Tulenko, S. R. Phillpot and S. B. Sinnott, "Energetics of Intrinsic Point Defects in Uranium Dioxide from Electronic Structure Calculations". J. Nucl. Mater. 384, 61-69 (2009).

- T. Watanabe, B. Ni, S. R. Phillpot, P. K. Schelling and P. Keblinski, "Thermal Conductance Across Grain Boundaries in Diamond from Molecular Dynamics Simulation":, J. Appl. Phys. 102063503 (2007).

- D. A. Vega, T. Watanabe, S. B. Sinnott, S. R. Phillpot and J. S. Tulenko, "Towards and Atomistically Informed Fuel Performance Code: Thermal Properties using 
FRAPCON and Molecular Dynamics Simulation", Nuclear Technology (in press; to be published March 2009).

- T. Watanabe, S. Srivilliputhur, P. K. Schelling, J. S. Tulenko, S. B. Sinnott and S. R. Phillpot, "Thermal Transport in Off-stoichiometric Uranium Dioxide by Atomic Level Simulation", J. Am. Ceram. Soc. (in press).

- D. S. Aidhy, P. C. Millett, D. Wolf, S. R. Phillpot and H. Huang, "Kinetically-driven Point-defect Clustering in Irradiated MgO by Molecular Dynamics Simulation", Scripta Materialia (in press).

- H. J. Seifert, P. Nerikar, H. L. Lukas, "Thermodynamic Assessment of the Ce-O System in Solid State from 60-67 mol\% O”, Int. J. Mater. Res., 97 744-752 (2006)

\section{Reviewed Proceedings:}

- H.J. Seifert, P. Nerikar, H.L. Lukas, "Thermodynamic Phase Modeling in the Ce-O System, ECS Transactions", 1 3-11 (2006).

\section{Reports:}

- P. Nerikar, H. J. Seifert, P. Perrot: The Iron-Oxgen-Uranium System, In: G. Effenberg, S. Ilyenko (eds.) Landolt-Börnstein, New Series IVI11C4 (2007), Ternary Alloy Systems - Phase Diagrams, Crystallographic and Thermodynamic Data: NonFerrous Metal Systems, Part 4: Selected Nuclear Materials and Engineering Systems, p. 312-319.

- P. Nerikar, H.J. Seifert, The Oxgen-Plutonium-Zirconium System, In: G. Effenberg, S. Ilyenko (eds.) Landolt-Börnstein, New Series IVI11C4 (2007), Ternary Alloy Systems - Phase Diagrams, Crystallographic and Thermodynamic Data: Non-Ferrous Metal Systems, Part 4: Selected Nuclear Materials and Engineering Systems, p. 416424.

\section{Invited Presentations:}

- H.J. Seifert: "Computational Thermodynamics of Nuclear Fuels and their Surrogates", Workshop on Materials Models and Simulations for Nuclear Fuels (MMSNF-3, Washington D.C., Nov. 18-19, 2004 (Organizers: Marius Stan, Los Alamos National Laboratory, and James S. Tulenko, University of Florida).

- P. Nerikar and S.B. Sinnott "Determination of Point Defect Formation Energies in Uranium Oxide" Computational Materials Science Network (CMSN) Symposium in Salt Lake City, Utah, September 13-14, 2007.

- T. Watanabe, P. Shukla, J, Nino, S. B. Sinnott, D. Vega, J. S. Tulenko, R. W. Grimes, S. R. Phillpot, Thermal Transport in Nuclear Fuels: Insights from Simulation 2007 Annual Meeting of the DOE-CMSN Project on Multiscale Simulation of Thermo - 
mechanical Processes in Irradiated Fission-Reactor Materials, Salt Lake City, UT, September 2007

- S. R. Phillpot, "Effects of Microstructure on Thermal Transport: Insights from Simulation", Idaho National Laboratory, August 2006.

- S. R. Phillpot, Interfacial Thermal Transport: Insights from Simulation, Molecular Modeling 2006, Perth Australia, April 2006.

- Taku Watanabe, Dilpuneet Aidhy, Tepan Desai, Dieter Wolf and Simon Phillpot, Radiation Damage in $\mathrm{UO}_{2}$ by Molecular Dynamics Simulation, CAARI,

- Simon R. Phillpot, The Role of Microstructure and Defects on Thermal Transport in $\mathrm{UO}_{2}$ and Related Materials, Workshop on Microstructire in Materials, Santa Fe NM, September 2008

- Simon R. Phillpot, Taku Watanabe, Dilpuneet Aidhy, Pankaj Nerikar and James S. Tulenko, Microscopic Processes in Oxide Fuels: Insights from Simulation, Symposium R: Materials for Future Fusion and Fission Technologies, Fall MRS Meeting, Boston MA, Dec. 2008

\section{Contributed Oral Presentations:}

- P. Nerikar, T. Watanabe, S.R. Phillpot and S.B. Sinnott, "Investigation of Point defect Formation and Migration in Uranium Oxide", $32^{\text {nd }}$ International Cocoa Beach Conference and Exposition on Advanced Ceramics \& Composites sponsored by American Ceramic Society (ACerS), Daytona Beach, Florida, January 27-February 1, 2008.

- P. Nerikar, T. Watanabe, J.S. Tulenko, S.R. Phillpot, and S.B. Sinnott, "Combined First Principles and Thermodynamic Calculation of Defect Formation Energies in $\mathrm{UO}_{2}$, , 2008 MRS Fall Meeting, Boston, MA, December 1-5, 2008.

- Taku Watanabe, Priyank Shukla, James Tulenko, Juan Nino, Susan Sinnott, and Simon R. Phillpot, "Thermal Transport in Nuclear Fuel: Insights from Simulation", Oral presentation, , ITCC29-ITES17 Session B26 Nuclear, Birmingham AL, June 27, 2007.

- T. Watanabe, S. R. Phillpot, S. B. Sinnott, J. S. Tulenko, S. Srivilliputhur, B. Uberuaga, M. Stan, S. Maloy, R. W. Grimes. Molecular Dynamics Simulation of Thermal Transport and Radiation Damage in $\mathrm{UO}_{2}$, TMS Spring Meeting, Orlando FL March 2007

- S. R. Phillpot, T. Watanabe, P. Shukla, S. B. Sinnott and J. C. Nino, J. S. Tulenko and Robin W. Grimes, "Thermal Transport in Nuclear Fuels by Atomic-Level Simulation", The Fifth Materials Models and Simulations for Nuclear Fuels Workshop (MMSNF-5), June 2006.

Posters: 
- Taku Watanabe, Priyank Shukla, James Tulenko, Juan Nino, Susan Sinnott, and Simon R. Phillpot "Thermal Transport of Selected Oxide Ceramics for Nuclear Fuel Application by Molecular Dynamics Simulation", Florida Society for Materials Simulation, University of South Florida, June 8, 2007.

- Pankaj Nerikar, Taku Watanabe, Simon R. Phillpot and Susan B. Sinnott, "AbInitio and Semi Empirical Calculations of Uranium Oxide", AVS 54th International Symposium in Seattle, WA, October 14-19, 2007

- Pankaj Nerikar, Taku Watanabe, Simon R. Phillpot and Susan B. Sinnott, "Point defect Formation and Migration in Uranium oxide", FL AVS-FSM-ASA Joint Symposium in Orlando, Florida, March 10-11, 2008.

- P. Nerikar and S.B. Sinnott, "Ab-Initio Calculations of Defect Formation Energies in Uranium Oxide", FL AVS-FSM-ASA March 2006 Joint Symposium in Orlando, Florida, March 12-13, 2007.

- P. Nerikar, T. Watanabe, S.R. Phillpot and S.B. Sinnott, "Ab-Initio and Semi Empirical Calculations of Uranium Oxide", Gordon Research Conference on SolidState Studies in Ceramics in Andover, New Hampshire, August 5-10, 2007.

\section{Student Education and Theses}

- Taku Watanabe - thermal transport properties of $\mathrm{UO}_{2}$ (Ph.D, Spring 2008; currently postdoc at Georgia Tech)

- Daniel Vega - first-principles input to FRAPCON (M.S, Summer 2008; now working at DOE-NE)

- Pankaj Nerikar - phase diagrams and point defect thermodynamics (Ph.D projected Spring 2009)

- Dilpuneet Aidhy - radiation damage in nuclear fuels (Ph.D. projected Spring 2009)

- Christian Acosta - first principles input to FRAPCON (M.S., Summer 2008) 
References:

${ }^{1}$ A. o. t. S. C. o. t. A. N. Society, "Method for Calculating the Fractional Release of Volatile Fission Products from Oxide Fuel," Report No. ANSI/ANS-5.4-1982 (1982).

${ }^{2}$ M. P. Bohn, "FRACAS: A Subcode for the Analysis of Fuel Pellet-Cladding Mechanical Interaction," Report No. TREE-NUREG-1028 (1977).

${ }^{3}$ D. L. Hagrman, A. Reymann, and R. E. Mason, MATPRO-Version 11 (Revision 1):

A Handbook of Materials Properties for Use in the Analysis of Light Water Reactor Fuel Rod Behavior, Vol. NUREG/CR-0497, Rev. 1 ed. (1980).

${ }^{4}$ K. Yamada, K. Kurosaki, M. Uno, and S. Yamanaka, Journal Of Alloys And Compounds 307, 10-16 (2000).

${ }^{5}$ M. Abramowski, R. W. Grimes, and S. Owens, Journal of Nuclear Materials 275, 12-18 (1999).

${ }^{6}$ G. Busker, Ph.D Thesis, Imperial College of Science, Technology, and Medicine, 2002.

${ }^{7}$ Y. Ida, Physics Of The Earth And Planetary Interiors 13, 97-104 (1976).

${ }^{8}$ D. Wolf, P. Keblinski, S. R. Phillpot, and J. Eggebrecht, Journal of Chemical Physics 110, 8254-8282 (1999).

${ }^{9}$ P. K. Schelling, S. R. Phillpot, and D. Wolf, Journal of the American Ceramic Society 84, 1609-1619 (2001).

10 J. D. Gale, Journal of the Chemical Society-Faraday Transactions 93, 629-637 (1997).

11 J. D. Gale and A. L. Rohl, Molecular Simulation 29, 291-341 (2003).

${ }^{12}$ K. Govers, S. Lemehov, and M. Verwerft, Journal of Nuclear Materials 266, 161177 (2007).

${ }^{13}$ J. K. Fink, Journal of Nuclear Materials 279, 1-18 (2000).

${ }^{14}$ N. W. Ashcroft and D. N. Mermin, Solid State Physics, College ed. (Saunders College Publishing, 1976).

${ }^{15}$ C. Kittel, Introduction to Solid State Physics, 7 ed. (John Wiley \& Sons, Inc., 1996).

${ }^{16} \mathrm{G}$. Leibfried and E. Schloemann, Math. Physik. K1 Ila, 71 (1954).

${ }^{17}$ P. G. Klemens, Thermal Conductivity and Lattice Vibrational Modes, Vol. 7 (1954). 
${ }^{18}$ P. Jund and R. Julien, Physical Review B 59, 13707 (1999).

${ }^{19}$ P. K. Schelling and S. R. Phillpot, Journal of the American Ceramic Society 84, 2997 (2001).

${ }^{20}$ P. K. Schelling, S. R. Phillpot, and K. Keblinski, Physical Review B 65, 144306 (2002).

${ }^{21}$ J. K. Fink, Int. J. Thermophys. 3, 165 (1982).

${ }^{22}$ H. Yoshida, K. Yokoyama, N. Shibata, Y. Ikuhara, and T. Sakuma, Acta Materialia 52, 2349-2357 (2004).

${ }^{23}$ P. Keblinski, D. Wolf, S. R. Phillpot, and H. Gleiter, Journal Of Materials Research 13, 2077-2099 (1998).

${ }^{24}$ P. Shukla, T. Watanabe, J. C. Nino, J. Tulenko and S. R. Phillpot, Journal of Nuclear Materials, submitted for publication.

${ }^{25}$ C. W. Nan and R. Birringer, Physical Review B 57, 8264-8268 (1998).

${ }^{26}$ H. S. Yang, G. R. Bai, L. J. Thompson, and J. A. Eastman, Acta Materialia 50, 2309-2317 (2002).

27 J. Amrit, Journal of Physics D-Applied Physics 39, 4472-4477 (2006).

${ }^{28}$ R. J. Stevens, L. V. Zhigilei, and P. M. Norris, International Journal of Heat and Mass Transfer 50, 3977-3989 (2007).

${ }^{29}$ D. G. Cahill, W. K. Ford, K. E. Goodson, G. D. Mahan, A. Majumdar, H. J. Maris, R. Merlin, and S. R. Phillpot, Journal of Applied Physics 93, 793-818 (2003).

30 J. M. Ziman, Electrons and Phonons (Oxford University Press, London, UK, 1960).

${ }^{31}$ P. W. Anderson, B. I. Halperin, and C. M. Varma, Philosophical Magazine 25, 1-9 (1971).

${ }^{32}$ C. Kittel, Physical Review 75, 972-974 (1949).

${ }^{33}$ G. A. Slack, Solid State Physics, Vol. 34 (Academic Press, New York, 1979).

${ }^{34}$ D. G. Cahill and R. O. Pohl, Solid State Communications 70, 927-930 (1989).

${ }^{35}$ P. B. Allen, J. L. Feldman, J. Fabian, and F. Wooten, Philosophical Magazine BPhysics of Condensed Matter Statistical Mechanics Electronic Optical and Magnetic Properties 79, 1715-1731 (1999). 
${ }^{36}$ J. L. Feldman, M. D. Kluge, P. B. Allen, and F. Wooten, Physical Review B 48, 12589-12602 (1993).

${ }^{37}$ C. R. A. Catlow, Proceedings of the Royal Society of London Series aMathematical Physical and Engineering Sciences 353, 533-561 (1977).

${ }^{38}$ C. Ronchi and G. J. Hyland, Journal of Alloys And Compounds 213, 159-168 (1994).

${ }^{39}$ P. G. Lucuta, H. Matzke, and I. J. Hastings, Journal of Nuclear Materials 232, 166180 (1996).

${ }^{40} \mathrm{~S}$. Yamasaki, T. Arima, K. Idemitsu, and Y. Inagaki, International Journal of Thermophysics 28, 661-673 (2007).

${ }^{41}$ D. G. Cahill, S. K. Watson, and R. O. Pohl, Physical Review B 46, 6131-6140 (1992).

42 J. Callaway, Physical Review 113, 1046-1051 (1959).

${ }^{43}$ P. G. Klemens, Proceedings of the Physical Society of London Section A 68, 11131128 (1955).

44 J. F. Ziegler, J. P. Biersak, and U. Littmark, The Stopping Range of lons in Solids, Pergamon (1985).

45 J. L. Whitton and H. Matzke, Canadian Journal of Physics 44, 2905-\& (1966).

${ }^{46}$ Y. H. Hu and S. B. Sinnott, Journal of Computational Physics 200, 251-266 (2004).

${ }^{47}$ S. A. Adelman and J. D. Doll, Journal of Chemical Physics 64, 2375-2388 (1976).

${ }^{48}$ L. R. Corrales, A. Chartier, and R. Devanathan, Nuclear Instruments \& Methods in Physics Research Section B-Beam Interactions with Materials and Atoms 228, 274281 (2005).

${ }^{49}$ N. Saunders and A. P. Miodownik, CALPHAD (Calculation of phase diagrams): $A$ comprehensive guide, Vol. 1 (Pergamon, Oxford, UK, 1998).

${ }^{50}$ H. J. Seifert and F. Aldinger, Zeitschrift fuer Metallkunde 87, 2-13 (1996).

${ }^{51}$ H. J. Seifert, B. Sundman, and F. Aldinger, Zeitschrift fuer Metallkunde 92, 513562 (2001).

${ }^{52}$ B. Sundman, B. Jansson, and J.-O. Andersson, CALPHAD 9, 153-190 (1985). 
53 J.-O. Andersson, T. Helander, L. Hoeglund, P. Shi, and B. Sundman, CALPHAD

26, 273-312 (2002).

${ }^{54}$ H. L. Lukas and S. G. Fries, Journal of Phase Equilibria 13, 532-541 (1992).

${ }^{55}$ W. L. Lyon and W. E. Baily, Journal of Nuclear Materials 22, 332-\& (1967).

56 "The plutonium-oxygen and uranium-plutonium-oxygen systems: a thermodynamic assessment," (1967).

${ }^{57}$ C. Affortit and J. Marcon, Rev. Int. Hautes Temp. Refract. 7, 236 (1970).

${ }^{58}$ M. Beauvy, Journal of Nuclear Materials 188, 232-238 (1992).

${ }^{59}$ D. G. Clifton, iLA-4749-MS, 28 (1971).

${ }^{60}$ R. L. Gibby, HEDL-TME-73-19 (1973).

${ }^{61}$ R. L. Gibby, L. Leibowitz, J. F. Kerrisk, and D. G. Clifton, Journal of Nuclear Materials 50, 155-161 (1974).

${ }^{62}$ R. Kandan, R. Babu, K. Nagarajan, and P. R. Vasudeva Rao, Journal of Nuclear Materials 324, 215-219 (2004).

${ }^{63}$ L. Leibowitz, D. F. Fischer, and M. G. Chasanov, Journal of Nuclear Materials 42, 113-116 (1972).

${ }^{64}$ L. Leibowitz, D. F. Fischer, and M. G. Chasanov, (1974).

${ }^{65}$ A. E. Ogard and J. A. Leary, Vienna, 1967, p. 651.

${ }^{66}$ E. H. P. Cordfunke and R. J. M. Konings, Thermochemical Data for Reactor Materials and Fission Products (North-Holland, Amsterdam, 1990).

${ }^{67}$ MATPRO, "MATPRO - A library of materials properties for light water reactor accident analysis," (1997).

68 J. J. Carbajo, G. L. Yoder, S. G. Popov, and V. K. Ivanov, Journal of Nuclear Materials 299, 181-198 (2001).

${ }^{69}$ W. Breitung and K. O. Reil, Nuclear Science and Engineering 105, 205-217 (1990).

${ }^{70}$ R. W. Ohse and W. M. Olson, 1970 (American Institute of Mining, Metallurgical and Petroleum Engineers, New York), p. 743.

${ }^{71}$ J. E. Battles, W. A. Shinn, P. E. Blackburn, and R. K. Edwards, 1970 (American Institute of Mining, Metallurgical and Petroleum Engineers, New York), p. 733. 
${ }^{72}$ G. Dean, J. C. Boivineau, P. Chereau, and J. P. Marcon, 1970 (American Institute of Mining, Metallurgical and Petroleum Engineers, New York), p. 753.

${ }^{73} \mathrm{G}$. R. Chilton and I. A. Kirkham, in The determination of oxygen potentials of hyperstoichiometric U-Pu dioxides in the temperature range 1500-1850K, Baden Baden, 1975 (North-Holland Publishing Company, Amsterdam).

${ }^{74} \mathrm{G}$. R. Chilton and R. K. Edwards, in Oxygen potentials of $U_{0.77} P u_{0.23} \mathrm{O}_{2 \pm x}$ in the temperature range $1250-1550^{\circ} \mathrm{C}$, Juelich,Germany, 1980 (International Atomic Energy Agency, Vienna), p. 357-367.

${ }^{75}$ R. E. Woodley, Journal of the American Ceramic Society 56, 116 (1973).

${ }^{76}$ R. E. Woodley, Journal of Nuclear Materials 74, 290-296 (1978).

${ }^{77}$ R. E. Woodley, Journal of Nuclear Materials 96, 5-14 (1981).

${ }^{78}$ R. E. Woodley and M. G. Adamson, Journal of Nuclear Materials 82, 65-75 (1979).

${ }^{79}$ M. H. Rand and T. L. Markin, 1968 (IAEA, Vienna), p. 637.

${ }^{80}$ M. Tetenbaum, 1975 (IAEA, Vienna), p. 305.

${ }^{81}$ D. W. Green, J. K. Fink, and L. Leibowitz, High Temp.-High Pr., 205 (1983).

${ }^{82}$ R. Viswanathan and M. V. Krishnaiah, Journal of Nuclear Materials 294, 69-76 (2001).

${ }^{83}$ P. Y. Chevalier and E. Fischer, Journal of Nuclear Materials 257, 213-255 (1998).

${ }^{84} \mathrm{H}$. Kinoshita, M. Uno, and S. Yamanaka, Journal of Alloys and Compounds 354, 129-137 (2003).

${ }^{85}$ M. Kurata, Calphad 23, 305-337 (1999).

${ }^{86}$ S. Yamanaka, H. Kinoshita, and K. Kurosaki, Journal of Nuclear Materials 326, 185-194 (2004).

${ }^{87}$ D. Labroche, O. Dugne, and C. Chatillon, Journal of Nuclear Materials 312, 21-49 (2003).

${ }^{88}$ D. Labroche, O. Dugne, and C. Chatillon, Journal of Nuclear Materials 312, 50-66 (2003).

${ }^{89}$ P. Y. Chevalier, E. Fischer, and B. Cheynet, Journal of Nuclear Materials 303, 1-28 (2002). 
${ }^{90}$ C. Gueneau, M. Baichi, D. Labroche, C. Chatillon, and B. Sundman, Journal of Nuclear Materials 304, 161-175 (2002).

${ }^{91}$ B. J. Lewis, W. T. Thompson, F. Akbari, D. M. Thompson, C. Thurgood, and J. Higgs, Journal of Nuclear Materials 328, 180-196 (2004).

92 S. L. Dudarev, D. N. Manh, A. P. Sutton, Philosophical Magazine B. 75 (1997) 613.

93 J. He, R. K. Behera, M. W. Finnis, X. Li, E. C. Dickey, S. R. Phillpot, S. B. Sinnott, Acta Materialia. 55 (2007) 4325.

${ }^{94}$ R. Grimes, W., Catlow, C., R., A., Philosophical Transactions of the Royal Society: Physical and Engineering Sciences 335, (1991) 609.

${ }^{95}$ D. R. Oboyle, F. L. Brown, and J. E. Sanecki, Journal of Nuclear Materials 29, (1969) 27. 